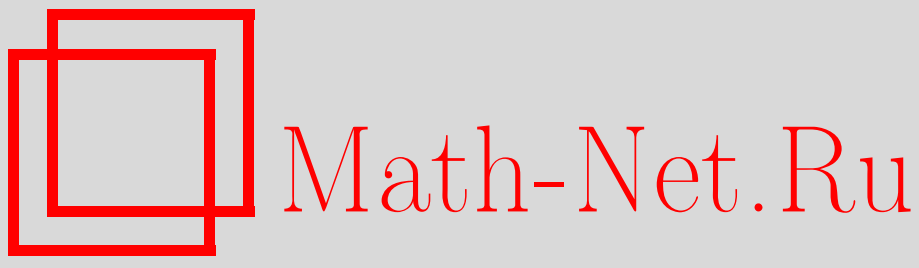

С. А. Мелихов, Об изотопической реализуемости отображений, пропущенных через гиперплоскость, Матем. сб., 2004, том 195, номер 8, 47-90

DOI: https://doi.org/10.4213/sm839

Использование Общероссийского математического портала Math-Net.Ru подразумевает, что вы прочитали и согласны с пользовательским соглашением http://www . mathnet.ru/rus/agreement

Параметры загрузки:

IP: 3.85 .7 .115

26 апреля 2023 г., $15: 54: 12$ 
УДК 515.1

\author{
С. А. Мелихов
}

\title{
Об изотопической реализуемости отображений, пропущенных через гиперплоскость
}

\footnotetext{
В работе исследуется проблема изотопической реализации, заключающаяся в вопросе об изотопической реализуемости заданного (непрерывного) отображения $f$, т.е. возможности равномерного приближения $f$ непрерьпным семейством вложений $g_{t}, t \in[0, \infty)$, при условии его дискретной реализуемости, т.е. наличии равномерного приближения последовательностью вложений $h_{n}, n \in \mathbb{N}$.

Для каждого $n \geqslant 3$ построено $f: S^{n} \rightarrow \mathbb{R}^{2 n}$, реализуемое дискретно, но не изотопически, которое, в отличие от всех таких ранее известных примеров, является локально плоским топологическим погружением. Для каждого $n \geqslant 4$ построено дискретно, но не изотопически реализуемое $f: S^{n} \rightarrow \mathbb{R}^{2 n-1} \subset \mathbb{R}^{2 n}$. Показано, что при $n \equiv 0,1(\bmod 4)$ изотопически реализуемо любое отображение $f: S^{n} \rightarrow \mathbb{R}^{2 n-2} \subset \mathbb{R}^{2 n}$, а при $n \equiv 2(\bmod 4)$ - всякое $f: S^{n} \rightarrow \mathbb{R}^{2 n-3} \subset$ $\mathbb{R}^{2 n}$. Если $n \geqslant 13$ и $n+1$ не является степенњ двойки, изотопически реализуется произволњное $f: S^{n} \rightarrow \mathbb{R}^{5[n / 3]+3} \subset \mathbb{R}^{2 n}$.

Основные результаты посвящены задаче изотопической реализации для отображений $S^{n} \stackrel{f}{\longrightarrow} S^{n} \subset \mathbb{R}^{2 n}, n=2^{l}-1$. Установлено, что, если ее решение отрицательно, прообразы точек при отображении $f$ обладают некоторьп гомологическим свойством, связанным с действиями группы целых $p$-адических чисел. Решение положительно, если $f$ липшицево и его нить ван Кампена-Скопенкова имеет конечный порядок. В связи с доказательством вводятся функторы Ext $\square$ и

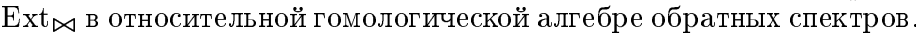

Библиографияя: 43 названия.
}

\section{Введение}

Непрерьвное отображение $f$ компактного полиэдра $X$ в PL-многообразие $Q$ (без края) назьвается дискретно реализуемым [1], если для каждого $\varepsilon>0$ оно $\varepsilon$-аппроксимируемо вложением, и изотопически реализуемым [2], если существует псевдоизотопия $H_{t}: Q \rightarrow Q, t \in I=[0,1]$ (т.е. изотопия с параметром $t \in[0,1)$, $H_{0}=\mathrm{id}_{Q}$, продолжающаяся до непрерывного отображения при $\left.t \rightarrow 1\right)$, переводящая в $f$ некоторое вложение $g$ (т.е. $H_{1} \circ g=f$ ). Очевидно, изотопическая реализуемость влечет дискретную. Вопрос о справедливости обратной импликации был поднят Е. В. Шепиным (см. [3; проблема 2]) и известен как проблема изотопической реализации.

Подробное введение и исторический обзор можно найти в [4] и [5] (см. также [3], [6]-[8]). В работе [7] доказано, что если $X^{n}$ - компактньй полиэдр, $Q^{m}$ - ориентируемое кусочно линейное многообразие, где $m>3(n+1) / 2$, и отображение $f: X \rightarrow Q$ дискретно реализуемо, то композиция $f$ и включения $Q \times\{0\} \hookrightarrow Q \times \mathbb{R}$

Работа выполнена при финансовой поддержке Российского фонда фундаменталњных исследований (гранты № № 99-01-00009, 02-01-00014). 
изотопически реализуема; согласно [5; добавление 1.2] размерностное ограничение можно ослабить до $m \geqslant 3(n+1) / 2, n \neq 1$. По этому поводу см. также начало $\S 2$ настоящей статьи, где приводится альтернативное доказательство в случае $Q=\mathbb{R}^{2 n-1}$. В [7] спрашивается, совпадают ли два понятия реализации для отображений $S^{n} \rightarrow \mathbb{R}^{m-1} \stackrel{\mathfrak{j}}{\hookrightarrow} \mathbb{R}^{m}$ и $S^{n} \rightarrow S^{n} \stackrel{\mathfrak{i}}{\hookrightarrow} \mathbb{R}^{2 n}$, где $\mathfrak{i}, \mathfrak{j}-$ стандартные включения.

На первый вопрос отрицательный ответ дается примерами 2 и $2^{\prime}$ в $\S 2$ (см. п. (в) теоремы 1 ниже). Заодно получается дискретно, но не изотопически реализуемое локально плоское топологическое погружение (см. пример 1 в $\S 2$ ). Вопрос [4; вопpoc I] о существовании такого отображения был мотивирован рассмотрением случая классических размерностей, в котором он, однако, до сих пор не поддается решению, приводя к серьезным нерешенным проблемам в теории (ручных) зацеплений [9] (см. также [8]). Пример 1 является, по мнению автора, эстетически наиболее удовлетворительным примером отображения, реализуемого дискретно, но не изотопически, хотя примеры из [4] проще с технической точки зрения.

Второй же вопрос (об отображениях, пропущенных через включение $n$-сферы в $\left.\mathbb{R}^{2 n}\right)$, которьй является основной мотивацией настоящей работы, полностью решить пока не удалось. Этот вопрос изучался также П. М. Ахметьевьм в [3], где приведен эскиз доказательства того факта, что в случае $n=4 k+1 \geqslant 5$ решение положительно, что является частным случаем следствия 2 ниже. Найти, по заданию рецензента, связь изложенных в этом эскизе идей (кроме тех, что явно содержатся уже в [10]) с методами настоящей работы автору не удалось.

ТЕОРема 1. Пусть $\beta(m)$ обозначает 2-примарную часть m, т.е. наибольшую степень двойки, на которую делится $m$.

(a) [3; док-во теоремы 1] (см. также [11; §3] и [10]) Любое отображсние $f: S^{n} \rightarrow \mathbb{R}^{2 n-\beta(n+1)} \subset \mathbb{R}^{2 n}, n \neq 2$, дискретно реализуемо.

(б) Изотопически реализуемо всякое $f: S^{n} \rightarrow \mathbb{R}^{2 n-k} \subset \mathbb{R}^{2 n}, n \neq 2$, где

$$
k= \begin{cases}2, & \text { если } n \equiv 0(\bmod 4) \\ 3, & \text { если } n \equiv 2(\bmod 4) ; \\ \beta(n+1), & \text { если } n \text { нечетно. }\end{cases}
$$

(в) Для кажсдого $n \geqslant 4$ существует отображсение $f: S^{n} \rightarrow \mathbb{R}^{2 n-1} \subset \mathbb{R}^{2 n}$, реализуемое дискретно, но не изотопически.

Утверждение п. (б) следует из теоремы 3.1. Неединообразный вид ответа в п. (б) объясняется тем, что имеются два независимых подхода, более простой из которых работает при ограничении $k=\max \{\beta(n+1), \beta(n+2)\}$, а более сложньй - при $k=\max \{\beta(n), \beta(n+1)\}+1 ;$ легко видеть, что в случае $n \not \equiv 2(\bmod 4)$ выигрывает первый подход, в противном случае - второй. Заметим также, что именно наличие этих двух подходов приводит далее к двум пунктам теоремы 2.

Отметим, что при $n=2$ утверждение п. (а) теоремы 1 не выполнено из-за того, что отображение $S^{2} \rightarrow \mathbb{R}^{3}$ может в общем положении иметь тройные точки. Более точно, двулистное накрытие над поверхностью Боя $S^{2} \rightarrow \mathbb{R P}^{2} \hookrightarrow \mathbb{R}^{3} \hookrightarrow \mathbb{R}^{4}$ не реализуемо дискретно в $\mathbb{R}^{4}[3]$; более тонкий пример, для которого дискретная реализуемость не выполнена даже “по модулю 2", приведен в [6] (см. также [12]).

СлеДСТВИЕ 1. Любое отображсение $S^{n} \rightarrow \mathbb{R}^{5[n / 3]+3} \subset \mathbb{R}^{2 n}$ изотопически реализуемо, если $n+1$ не является степенью двойки, $n \neq 2,4,6,9,10,12$. 
ДокаЗАТЕЛЬСтво. Предполагая, что $n+1=m * \beta(n+1)$, где $m \geqslant 3$, легко убедиться, что при $k \neq 4,6$ в условиях теоремы $1,(б)$ выполнено $k \leqslant[(n+1) / 3]$. Рассматривая отдельно случай $3 \nmid m$, эту оценку можно слегка улучшить, пожертвовав еше тремя размерностями.

СЛЕДСТВИЕ 2. Для любого отображсения $f: S^{n} \rightarrow S^{n}$ и любого топологического вложсения $\mathfrak{i}: S^{n} \hookrightarrow \mathbb{R}^{2 n}$ композичия $\mathfrak{i} \circ f$ изотопически реализуема при условии, что $n+1$ не является степенью двойки, $n \neq 2$.

ДокАЗАТЕЛьство. Если $\mathfrak{i}$ - стандартное вложение, утверждение является частньм случаем теоремы 1 . По теореме Зимана [13] любое PL-вложение i изотопно стандартному, и тем самым для него утверждение также выполнено. Если теперь $\mathfrak{i}$ - ТОР-вложение, в силу теоремы Эдвардса [14] существует псевдоизотопия $h_{t}$, переводящая на i некоторое PL-вложение j (см. [4; теорема 3.5a]), и искомая псевдоизотопия может быть получена диагональным образом из $h_{t}$ и псевдоизотопии, переводящей некоторое вложение на ј ○ $f$ (см. [4; $§ 4]$ ).

Заметим, что среди отображений $S^{n} \rightarrow \mathbb{R}^{2 n}$ те из них, образы которых содержатся в сфиере $S^{n} \subset \mathbb{R}^{n+1} \subset \mathbb{R}^{2 n}$, вызывают особый интерес в контексте вопросов о дискретной и изотопической реализуемости. Во-первых, они представляют собой первьй нетривиальный случай с точки зрения коразмерности, поскольку любое отображение $S^{n} \rightarrow S^{n-1} \subset \mathbb{R}^{2 n}$ изотопически реализуется распроектированием посредством совместного отображения ввиду того, что реализуемо изотопически постоянное отображение $S^{n} \rightarrow\{0\} \hookrightarrow \mathbb{R}^{n+1}$ в слой тривиального нормального расслоения к $S^{n-1}$ в пространстве $\mathbb{R}^{2 n}$. Немного более тонкие рассуждения позволяют уточнить оценку.

ПРЕДЛОЖЕНИЕ 1. (а) Всякое $S^{n} \stackrel{f}{\longrightarrow} \mathbb{R}^{n} \subset \mathbb{R}^{2 n}$ изотопически реализуемо.

(б) Отображсение $S^{n} \stackrel{f}{\longrightarrow} S^{n} \subset \mathbb{R}^{2 n}$ изотопически реализуемо при условии, что $f^{-1}(p)=\{p\}$ для некоторой $p \in S^{n}$.

ДокАЗАТЕльство. График Г ограничения $f$ из п. (б) на дополнение к $p$ лежит в произведении $\left(S^{n} \backslash\{p\}\right) \times\left(S^{n} \backslash\{p\}\right)$, которое можно отождествить с тотальным пространством $\mathbb{R}^{n} \times\left(S^{n} \backslash\{p\}\right)$ нормального расслоения к некомпактному подмногообразию $S^{n} \backslash\{p\} \subset S^{n} \subset \mathbb{R}^{2 n}$. Поскольку диаметр слоя этого расслоения стремится к нулю при удалении точки базы на бесконечность, Г имеет одноточечную компактификацию в топологии $\mathbb{R}^{2 n}$ с точкой $p$ в качестве короны. Следовательно, стандартное вложение $S^{n} \backslash\{p\}$ на Г продолжается до вложения $g$ сферы $S^{n}$ в тотальное пространство $T$ нормального расслоения к $S^{n} \subset \mathbb{R}^{2 n}$, композиция которого с проекцией $\pi$ на базу есть $f$. Остается сослаться на изотопическую реализуемость $T \stackrel{\pi}{\longrightarrow} S^{n} \subset \mathbb{R}^{2 n}$.

Для доказательства (а) рассмотрим путь $\varphi: I \rightarrow \mathbb{R}^{n}$ такой, что $\varphi(t) \in f\left(S^{n}\right)$, если и только если $t=0$. Зафиксировав какую-нибудь $p \in f^{-1}(\varphi(0))$ и отождествив в сфере $S^{n}$ все точки, равноудаленные от $p$ на расстояние не более $t$, получим гомотопию $h_{t}: S^{n} \rightarrow S^{n} \cup_{p=0} I$, где $h_{0}=\operatorname{id}_{S^{n}}, h_{t}^{-1}(t)=\{p\}$. Согласно п. (б) композиция $(f \cup \varphi) \circ h_{t}$ изотопически реализуема при $t>0$ (можно считать $\mathbb{R}^{n} \subset S^{n}$ ), причем ясно, что вложение $g=g(t)$ и псевдоизотопия $H_{s}=H_{s}(t)$ непрерьвно зависят от $t$. Более того, сушествует псевдоизотопия $G_{t}$ такая, что $g(1-t)=G_{t} \circ g(1)$. Значит, диагональная псевдоизотопия $F_{t}=H_{t}(1-t) \circ G_{t}$ переводит $g(1)$ на композицию $f$ и включения $S^{n} \subset \mathbb{R}^{2 n}$. 
Во-вторых, как ясно из [6], [15] (см. также [16]), рассмотрение отображений $S^{n} \rightarrow S^{n} \subset \mathbb{R}^{2 n-k}$ тесно связано с изучением итерированного гомоморфизма надстройки в гомотопических группах сффер, а также (ввиду итерационных возможностей отображений $S^{n} \rightarrow S^{n}$ ) допускает приложения к вложимости компактов [6]. Наконец отметим, что, как ясно из доказательства следствия 2, ответы на вопросы о дискретной и изотопической реализуемости композиции отображения $f: S^{n} \rightarrow S^{n}$ и (топологического) вложения $\mathrm{i}: S^{n} \hookrightarrow \mathbb{R}^{m}, m-n \geqslant 3$, не зависят от выбора вложения i. Поэтому корректно говорить о реализуемости $f$ в пространстве $\mathbb{R}^{m}$, что возвращает нас к исходной терминологии [1], [2]. Легко видеть, что композиция дискретно (изотопически) реализуемых в $\mathbb{R}^{m}$ отображений $S^{n} \rightarrow S^{n}$ дискретно (изотопически) реализуема в $\mathbb{R}^{m}$.

С помощью теоремы Адамса об инварианте Хопфа П.М. Ахметьеву удалось доказать, что любое отображение $\bar{f}: S^{n} \stackrel{k}{\longrightarrow} S^{n} \subset \mathbb{R}^{2 n}$ дискретно реализуемо при $n \neq 1,2,3,7$ [6] (см. альтернативные доказательства в [16], [11; §3]). При $n=1$ отображение $\bar{f}$ дискретно реализуемо, если и только если $\operatorname{deg}(f)=0, \pm 1[1]$. В случае $n=2$ Ахметьев недавно нашел элементарное доказательство дискретной реализуемости $\bar{f}$, основанное на методе М. Ямамото (см. [11]). При $n=3,7$ дискретная реализуемость $\bar{f}$ установлена в случае $\operatorname{deg}(f)=0, \pm 2, \pm 4$ или $2 k+1, k \in \mathbb{Z}[11]$. Для вопроса об изотопической реализуемости случай $n=2^{l}-1$ представляет особую сложность, поскольку в проблеме изотопической реализации ту роль, которую инвариант Хопфа играет в задаче дискретной реализации, занимает относительная версия (ср. гомоморфизмы $\rho_{n m}^{k}$ из [16]) второго инварианта Хопфа $H_{2}$ в смысле Уайтхеда-Джеймса (см. [15]), а для последнего удается применить лишь доказательство [10] легкой части (принадлежащей Адему [17]) теоремы Адамса (общий случай см. в [18]), работающее при ограничении $n \neq 2^{l}-1$.

Таким образом, размерностные ограничения в следствии 2, по-видимому, не удастся ослабить (или же доказать их необходимость) стандартными методами теории погружений без дальнейшего развития ее аппарата. Перейдем к формулировке результатов, полученных другими методами.

ОПРЕДЕЛЕния. Пусть $f: X \rightarrow Q$-непрерывное отображение. Замкнутое подмножество $\Sigma_{f}=\{(x, y) \mid x \neq y, f(x)=f(y)\}$ взрезанного квадрата $\tilde{X}=X \times$ $X \backslash \Delta_{X}$, где $\Delta_{X}=\{(x, x)\}$ - диагональ, инвариантно при свободной инволюции $\mathfrak{t}:(x, y) \leftrightarrow(y, x)$ на $\widetilde{X}$. Отметим, что компактность $\Sigma_{f}$ равносильна тому, что $f-$ (топологическое) погружение, а его пустота - тому, что $f$ - (топологическое) вложение; если $f$ - кусочно линейное отображение между полиэдрами, $\Sigma_{f}-$ подполиэдр $\widetilde{X}$, а если $f$ - отображение обшего положения между многообразиями, $\Sigma_{f}-$ подмногообразие $\widetilde{X}$.

В приложении к [5] определяются гомологические препятствия $\check{(}(f)$ и $o(f)($ а в § 1 настоящей статьи - их когомологические эквиваленты $\breve{\vartheta}(f)$ и $\vartheta(f))$ к дискретной и к изотопической реализуемости отображения $f: N^{n} \rightarrow M^{m}$ между ориентируемьпи кусочно линейньми многообразиями; приведем краткий набросок их построения. Препятствие $\check{(}(f)$ принимает значение в $(2 n-m)$-мерной группе локально конечных гомологий Александрова-Чеха $\sigma$-компакта $\Sigma_{f} / \mathrm{t}$ с некоторыми локальными коэффициентами, изоморфной обратному пределу локально конечных ${ }^{1}$ гомоло-

\footnotetext{
${ }^{1}$ Напомним (см. [5; приложение]), что локально конечные гомологии отличаются от обычных наличием циклов с некомпактными носителями (аналогично носителям коциклов в обычных когомологиях).
} 
гий полиэдральных окрестностей $\Sigma_{f} / \mathfrak{t}$ в $\widetilde{N} / \mathfrak{t}$, и определяется как нить из гомологических классов многообразий $\Sigma_{f_{i}} / \mathfrak{t}$, где $f_{i}$ - аппроксимации общего положения отображения $f$, в этих окрестностях $N_{i}$, объединенных с окрестностями $D_{i}$ бесконечности некомпактного многообразия $\widetilde{N} / \mathfrak{t}$. Препятствие $o(f)$ принимает значение в $(2 n-m)$-мерной группе локально конечных гомологий Стинрода-Ситникова $\sigma$-компакта $\Sigma_{f} / \mathfrak{t}$ с некоторыми локальными коэффициентами, изоморфной группе $(2 n-m+1)$-мерных локально конечных гомологий телескопа обратной последовательности окрестностей $N_{i}$, и определяется как гомологический класс многообразия $\bigcup \Sigma_{f_{t}} / \mathfrak{t}$, где $f_{t}: N \rightarrow M, t \in[0,1),-$ гомотопия обшего положения такая, что $f_{t} \rightarrow f$ равномерно при $t \rightarrow 1$, в телескопе обратной последовательности $N_{i} \cup D_{i}$.

ПрЕДЛОЖЕнИЕ 2. Пусть $\bar{f}: N^{n} \rightarrow Q^{2 n}$ - отображение между ориентируемыми кусочно линейными многообразиями, где $n \geqslant 3$ и $N$ компактно.

(a) [19], [7], [5] Отображсение $\bar{f}$ дискретно реализуемо, если и только если $\check{o}(\bar{f})=0$, и изотопически реализуемо, если и только если $о(\bar{f})=0$.

(б) Если $\bar{f}$ дискретно реализуемо и является композицией $N \stackrel{f}{\longrightarrow} M \subset Q$, где $Q=M \times \mathbb{R}^{k}, M^{2 n-k}$ - кусочно линейное многообразие, $k>0$, то класс $o(\bar{f})$ имеет порядок 2 и является образом о̆ $(f)$ при некотором гомоморфизме.

Доказательство п. (а) состоит, с учетом гомотопического критерия изотопической реализуемости (теорема 1.1), из стандартной теории препятствий (см. теорему 1.2) и стандартного перехода от когомологий к гомологиям (см. [5; приложение]). По модулю этого перехода первое утверждение п. (б) доказано в предложении 2.2 (другое доказательство - в замечании к лемме 3.3), а второе, играющее роль ключевого наблюдения в настоящей статье, - в предложении 6.4 (а также вытекает из леммы 3.3 , (a) и наблюдения 4.4 , что доставляет альтернативное определение интересуюшего гомоморфизма). Некоторые следствия второй части п. (б) установлены уже в теореме 2.1 (случай $m=2 n$ ) и в наблюдении 3.4 .

Теорема 2. Допустим, что композиция $\bar{f}: S^{n} \stackrel{f}{\longrightarrow} S^{n} \subset \mathbb{R}^{2 n}, n \geqslant 3$, реализуема дискретно, но не изотопически, и пусть $x \in S^{n}$ - любая точка, $P_{x}=f^{-1}(f(x))$.

(a) Eсли о̆ $(f)$ имеет конечный порядок, то компакт $P_{x}$ соленоидален, т.е. нетривиально ядро канонического эпиморфизма $\mathscr{F}: H_{0}\left(P_{x}\right) \rightarrow \check{H}_{0}\left(P_{x}\right)$ между нульмерными гомологиями Стинрода-Ситникова и Александрова-Чеха. Более того, это ядро содержит әлемент порядка 2.

(б) Образ индущированного включением гомоморфизма

$$
\widetilde{H}_{0}\left(P_{x}\right) \cong H_{0}^{\mathrm{lf}}\left(P_{x} \backslash\{x\}\right) \stackrel{i_{*}}{\longrightarrow} H_{0}^{\mathrm{lf}}\left(\Sigma_{f} / \mathfrak{t}\right)
$$

содержит әлемент порядка 2, принадлежсащий ядру

$$
\mathscr{F}: H_{0}^{\mathrm{lf}}\left(\Sigma_{f} / \mathfrak{t}\right) \rightarrow \check{H}_{0}^{\mathrm{lf}}\left(\Sigma_{f} / \mathfrak{t}\right)
$$

и не зависящий от х. Этот әлемент - не что иное, как $(\bar{f})$.

Доказательства когомологических версий пунктов приводятся в $\S \S 4-6$ и в начале $\S 4$ соответственно, формальная редукция к ним - в конце $\S 6$.

ЗАмечаниЕ. Из точной последовательности Милнора (см. [5; приложение]), связьваюшей гомологии Стинрода-Ситникова и Александрова-Чеха, вытекает, что всякий соленоидальный компакт имеет размерность не менее 1. 
Замечание. Сушествование отображений $S^{n} \rightarrow S^{n}$ без несоленоидальных прообразов точек представляется автору маловероятным. (Следует уточнить, что специалистам таким, как, например, Р. Эдвардс, неизвестно, существуют ли такие отображения, равно как и отображения $f: S^{n} \rightarrow S^{n}$ с $f^{-1}(x) \cong S^{1}$ для каждой $x \in S^{n}$.) В самом деле, известно, что факторпространство $S^{n}$ по свободному действию $p$-адического соленоида (если такое существует) имеет размерность не менее $n+1[20]$.

ЗАмечание (Е.В. Щепин). Прообраз хотя бы одной точки не является соленоидальньм, если $f$ липшицево или 1-мягкое (доказательство основано на доказательстве леммы Сарда, упрощенном с учетом того, что размерности образа и прообраза совпадают).

ЗАмечание (А.Н. Дранишников). Если прообраз каждой точки некоторого открытого множества $U \subset S^{n}$ гомеоморфен $p$-адическому соленоиду (который ацикличен $\bmod p$ ), по теореме Вьеториса-Бегля $f$ индуцирует изоморфизм

$$
H^{*}\left(S^{n}, S^{n} \backslash U ; \mathbb{Z} / p\right) \rightarrow H^{*}\left(S^{n}, S^{n} \backslash f^{-1}(U) ; \mathbb{Z} / p\right) .
$$

$\mathrm{B}$ частности, $\operatorname{deg} f \not \equiv 0(\bmod p)$. Правда, здесь не учтены такие, например, возможности: (i) прообраз каждой точки гомеоморфен $\ell$-адическому соленоиду, где $\ell=(2,3,5,7,11, \ldots)$; (ii) $S^{n}=T_{3} \cup T_{5}$, где $T_{p}$ - всюду плотное множество, прообраз каждой точки которого гомеоморфен $p$-адическому соленоиду.

ЗАмЕчАнИЕ. Из второго утверждения п. (а) следует, что в его условиях 2-адический соленоид не может быть прообразом никакой точки, поскольку в его одномерных гомологиях нет элементов порядка 2.

ЗАмЕчАниЕ. Следующий пример показывает, что существование отображений $S^{n} \rightarrow S^{n}$, удовлетворяющих заключению первого утверждения п. (б) для каждой $x \in S^{n}$, является весьма правдоподобным. Для проекции $f: \Sigma_{p} \rightarrow S^{1}$ $p$-адического соленоида, $p>2$, на окружность и любой $x \in S^{1}$ образ индуцированного включением гомоморфизма $\breve{H}_{0}\left(f^{-1}(x)\right) \cong H_{0}\left(f^{-1}(x)\right) \stackrel{i_{*}}{\longrightarrow} H_{0}\left(\Sigma_{p}\right)$ содержит элемент порядка 2 , принадлежащий ядру $\mathscr{F}: H_{0}\left(\Sigma_{p}\right) \rightarrow \check{H}_{0}\left(\Sigma_{p}\right)$. В самом деле, $i_{*}-$ эпиморфизм, поскольку по аксиоме вырезания

$$
H_{0}\left(\Sigma_{p}, f^{-1}(x)\right) \cong H_{0}(I \times C, \partial I \times C)=0
$$

где $C$ обозначает канторово множество. Между тем по формуле универсальных коэффициентов $H_{0}\left(\Sigma_{p}\right) \cong \mathbb{Z} \oplus \operatorname{Ext}\left(\mathbb{Z}_{(p)}, \mathbb{Z}\right)$, где $\mathbb{Z}_{(p)}$ - локализация целых чисел в $p$, изоморфная прямому пределу спектра из групп $H^{1}\left(S^{1}\right)$ и гомоморфизмов, индуцированных $p$-листньм накрытием. Но согласно упражнению из [21] $\operatorname{Ext}\left(\mathbb{Z}_{(p)}, \mathbb{Z}\right) \cong \mathbb{Z}_{p} / \mathbb{Z}$, где $\mathbb{Z}_{p}$ обозначает группу целых $p$-адических чисел, содержашую $\mathbb{Z}\left[\frac{1}{2}\right] \subset \mathbb{Z}_{(p)}$ в качестве подгруппы. Два чисто геометрических описания изоморфизма $\widetilde{H}_{0}\left(\Sigma_{p}\right) \cong \mathbb{Z}_{p} / \mathbb{Z}$ приведены в [5; пример 4].

Известно, что $f: \Sigma_{p} \rightarrow S^{1}$ является главным $\mathbb{Z}_{p}$-расслоением, в частности, проекцией на пространство орбит канонического свободного действия $\mathbb{Z}_{p}$ на $\Sigma_{p}$. Этим мотивируется 
ПроБлема 1. Если $\bar{f}: S^{n} \stackrel{f}{\longrightarrow} S^{n} \subset \mathbb{R}^{2 n}$ реализуемо дискретно, но не изотопически, верно ли, что отображение $f^{(2)}: \Sigma_{f} / \mathfrak{t} \rightarrow S^{n}$, заданное по формуле $\{x, y\} \mapsto f(x)=f(y)$, совпадает с проекцией на пространство орбит некоторого эффективного действия на $\Sigma_{f} / \mathfrak{t}$ канторовой группь, являющейся нетривиальным расширением $\widetilde{H}_{0}^{\mathrm{lf}}\left(\Sigma_{f} / \mathfrak{t}\right)$ посредством $\mathbb{Z}$, и согласованного слевым действием $\widetilde{H}_{0}^{\text {lf }}\left(\Sigma_{f} / \mathfrak{t}\right)$ на себе?

ЗАмЕчАниЕ. Несложно показать, что любой элемент ядра канонического эпиморфизма $\mathscr{F}$ имеет бесконечную высоту по любому основанию $p$, делящему порядок элемента, если тот конечен (достаточно рассмотреть последовательность Милнора, см. [5; приложение], с коэффициентами в $\mathbb{Z} / p^{h+1}$, где $h$-высота). В частности, всякий элемент порядка 2 в этом ядре четен и, стало быть, потеряется, если привести коэффициенты по модулю 2. Поскольку - -произведение нечетных классов может оказаться четным, нам потребуется систематически различать элемент поря дка 2, даже если он нечетен, в когомологиях с цельми (быть может, локальными) коэффициентами и соответствующий ему элемент в когомологиях по модулю 2. В частности, для одномерного векторного расслоения мы различаем первый класс Штифеля-Уитни $w_{1}$, принимающий значение в одномерных когомологиях по модулю 2 , и класс Эйлера $e$, являющийся элементом порядка 2 (либо 1) в локальных целочисленных одномерных когомологиях.

ПроБлема 2. Существует ли отображсение $S^{n} \stackrel{f}{\longrightarrow} S^{2 n-k} \subset \mathbb{R}^{2 n}$, реализуемое дискретно, но не изотопически, и такое, что

(a) $\operatorname{dim} \Sigma_{f}=k ?$

(б) о̆ $(f)$ имеет бесконечный порядок?

(в) существует локальная изотопическая реализация $f$ (т.е. регулярная гомотопия $F: S^{n} \times[0,1) \rightarrow S^{2 n-k} \times[0,1)$ такая, что $F \cup(f \times 1): S^{n} \times I \rightarrow$ $S^{2 n-k} \times I$ непрерывно), ограничение которой на $S^{n} \times[0,1-\varepsilon]$ распроектируется в изотопию $S^{n} \times[0,1-\varepsilon] \hookrightarrow \mathbb{R}^{2 n} \times[0,1-\varepsilon]$ для всякого $\varepsilon>0 ?$

Неравенство $\operatorname{dim} \Sigma_{f} \leqslant k$ следует из предложения $2,(б)$, но неясно, может ли оно быть усилено до $\operatorname{dim} \Sigma_{f} \leqslant k+1$, из чего вытекал бы отрицательный ответ на вопрос проблемы 1. Такое усиление имеет место, если ответ на вопрос (б) отрицателен (элемент конечного порядка может быть получен из гомологий на единицу большей размерности с помощью гомоморфизма Бокштейна, причем ввиду конечности порядка эти прообразы можно объединить в нить, см. доказательство предложения 4.6). Однако вопрос (б) пока не удалось решить даже в случае $k=1$, хотя в $\S 5$ требуемьй пример построен на уровне $\Sigma_{f}$ (являющегося одномерньм), неясно лишь, реализуется ли этот компакт некоторым отображением $f$. Этот вопрос интересен также тем, что наличие таких отображений позволило бы (уже при $k=1)$ получить геометрическую интерпретацию возможности “нерасшепимости на бесконечности" короткой точной последовательности обратных последовательностей конечно порожденных абелевых групп (см. §5). Конечно, вопрос (б) наиболее важен с точки зрения отображений $S^{n} \rightarrow S^{n} \subset \mathbb{R}^{2 n}$, как показывает сравнение двух пунктов теоремы 2.

Ответ на вопрос (в) отрицателен в случае $k=1$. В самом деле, для каждого $\varepsilon>0$ задано эквивариантное отображение $\Sigma_{F} \cap S^{n} \times[0,1-\varepsilon] \rightarrow S^{0}$, поэтому ввиду конечности множества эквивариантных гомотопических классов таких 
отображений существует эквивариантное отображение $\Sigma_{F} \rightarrow S^{0}$, доставляющее изотопическую реализацию $F$ (по лемме 4.2 и [4; теорема 1.12]).

\section{§1. Первое препятствие к изотопической реализуемости}

ОПРЕДЕЛЕНИЯ. Отображение $\Phi: X \times X \rightarrow Q \times Q$ называется изовариантным, если оно эквивариантно относительно переставляющих сомножители инволюций и $\Phi^{-1}\left(\Delta_{Q}\right)=\Delta_{X}$. Таково, например, отображение $f^{2}: X \times X \rightarrow Q \times Q$, где $f: X \hookrightarrow Q$-произвольное вложение. Полезно иметь в виду случай $Q=\mathbb{R}^{m}$, когда эквивариантные гомотопические эквивалентности $\widetilde{\mathbb{R}^{m}} \rightarrow \nabla_{\mathbb{R}^{m}} \backslash\{0\} \rightarrow S^{m-1}$, где $\nabla$ обозначает антидиагональ $\{(x,-x)\}$, сводят изовариантные отображения $X \times X \rightarrow Q \times Q$ к эквивариантным $\widetilde{X} \rightarrow S^{m-1}$, где $\mathbb{Z} / 2$ действует перестановкой сомножителей $\mathfrak{t}$ на $\widetilde{X}$ и антиподальной инволюцией $\mathfrak{s}$ на $S^{m-1} \dot{\tilde{f}}$ Для произвольного отображения $f: X \rightarrow \mathbb{R}^{m}$ его отображсние моментов $\tilde{f}: \widetilde{X} \backslash \Sigma_{f} \rightarrow S^{m-1}$ определяется по формуле

$$
(x, y) \mapsto \frac{f(x)-f(y)}{\|f(x)-f(y)\|} .
$$

TЕорема 1.1. Пусть $X^{n}$ - компактный полиэдр, $Q^{m}$ - кусочно линейное многообразие без края, $m \geqslant 3(n+1) / 2, u f: X \rightarrow Q$ - непрерьвное отображсение.

(a) [19], [4] Отображение $f$ дискретно реализуемо, если и только если $f^{2}$ аппроксимируется изовариантныци отображениями. Более того, $\forall \varepsilon>0$ $\exists \delta>0$ такое, что любое $\delta$-близкое $\kappa f^{2}$ изовариантное отображение $\varepsilon$-гомотопно в классе изовариантных отображений квадрату некоторого вложения.

(б) [4], [5] При $n \neq 1$ отображсение $f$ изотопически реализуемо, если и только если существует гомотопия $\Phi_{t}: X \times X \rightarrow Q \times Q$ такая, что $\Phi_{0}=f^{2}$ $u \Phi_{t}$ изовариантно при $t>0$. Более того, $\forall \varepsilon>0 \exists \delta>0$ такое, что любое вложение $g: X \hookrightarrow Q$, для которого $f^{2}$ и $g^{2}$ соединяются $\delta$-гомотопией, состоящей из изовариантных отображений при $t>0$, переводится на $f$ некоторой є-псевдоизотопией.

Далее напоминаются необходимые определения и одновременно фиксируются обозначения, которые будут использоваться на протяжении всей статьи.

Эквивариантные когомологии. Пусть $K$ - локально компактньй полиэдр, на котором задана свободная кусочно линейная инволюция $\mathfrak{t}$, и $L$ - его инвариантный подполиэдр (возможно, пустой). Группы целочисленных когомологий $H^{i}(K / \mathfrak{t}, L / \mathfrak{t} ; \mathbb{Z})$ мы будем обозначать через $H_{\mathrm{eq}}^{i}(K, L)$, если $i$ нечетно, и через $H_{\text {skew }}^{i}(K, L)$, если $i$ четно; при противоположной четности $i$ эти обозначения относятся к когомологиям $H^{i}\left(K / \mathfrak{t}, L / \mathfrak{t} ; \mathscr{O}_{\mathbb{Z}_{\mathrm{T}}}\right)$ с целочисленными локальными коэффициентами, скрученньми вдоль петель в $K / \mathfrak{t}$, не поднимающихся в $K$ (заметим, что это не совсем согласуется с обозначениями [7]). В терминологии [22] локально постоянный пучок $\mathscr{O}_{\mathbb{Z}_{\mathrm{T}}}-$ это 0 -мерный пучок Лере отображения $K \rightarrow K /$ t. Таким образом,

$$
\begin{aligned}
H_{\text {eq }}^{m}(K, L) & \simeq H^{m}\left(K / \mathfrak{t}, L / \mathfrak{t} ; \mathscr{O}_{\mathbb{Z}_{\mathrm{T}}}^{\otimes m+1}\right), \\
H_{\text {skew }}^{m}(K, L) & \simeq H^{m}\left(K / \mathfrak{t}, L / \mathfrak{t} ; \mathscr{O}_{\mathbb{Z}_{\mathrm{T}}}^{\otimes m}\right) .
\end{aligned}
$$


Несложно видеть, что $O_{\mathbb{Z}_{\mathrm{T}}}^{\otimes k}$ является ориентирующим пучком многообразий $\mathbb{R} P^{k-1}$ и $\Sigma_{f} / \mathfrak{t}$, где $f$ - отображение общего положения в коразмерности $k$ между ориентируемьми многообразиями (см. [5; приложение]).

Группы $H_{\text {eq }}^{m}(K, L)$ и $H_{\text {skew }}^{m}(K, L)$ иногда удобно считать определенными аналогично обычным целочисленным когомологиям $H^{m}(K, L)$ с тем отличием, что все построения производятся в некоторой эквивариантной триангулящии, а вместо коцепей рассматриваются лишш (косо)эквивариантные коцепи, т.е. такие коцепи $c$, что $c \circ \mathfrak{t}=(-1)^{m+1} c$ (соответственно $\left.c \circ \mathfrak{t}=(-1)^{m} c\right)$. В самом деле, имеют место изоморфизмы

$$
\begin{aligned}
H_{\mathrm{eq}}^{m}(K, L) & \simeq H_{\mathbb{Z} / 2}^{m}\left(K, L ; \mathbb{Z}_{\mathrm{T}}^{\otimes m+1}\right), \\
H_{\text {skew }}^{m}(K, L) & \simeq H_{\mathbb{Z} / 2}^{m}\left(K, L ; \mathbb{Z}_{\mathrm{T}}^{\otimes m}\right),
\end{aligned}
$$

где $\mathbb{Z}_{\mathrm{T}}$ обозначает $\mathbb{Z}[\mathbb{Z} / 2]$-модуль $\mathbb{Z}_{\mathrm{T}}=\mathbb{Z}[\mathbb{Z} / 2] /(\mathfrak{t}+1)$, соответствующий пучку $\mathscr{O}_{\mathbb{Z}_{\mathrm{T}}}$; определение эквивариантных когомологий напоминается в $[5$; приложение].

Когомологии неполиэдральных пар полиэдров. Пусть $O$ - объединение возрастающей цепочки $K_{0} \subset K_{1} \subset \cdots$ инвариантных подполиэдров $K$ (например, в качестве $O$ подходит любое инвариантное открытое подмножество $K$ или какого-нибудь его подполиэдра). Определим группу $H_{\pi}^{m}(K, O ; M)$ как $H_{\pi}^{m}(K \times[0,1)$, $U ; M)$, где $U$ - телескоп прямой последовательности $\left\{K_{i}\right\}$, т.е. объединение цилиндров $K_{i} \times\left[1-2^{i}, 1-2^{i+1}\right]$. Индуцированный включением $O \subset K$ гомоморфизм ограничения в когомологиях определяется как композиция $H_{\pi}^{m}(K ; M) \simeq$ $H_{\pi}^{m}(K \times[0,1) ; M) \rightarrow H_{\pi}^{m}(U ; M) \simeq H_{\pi}^{m}(O ; M)$. Ясно, что эти определения не зависят от выбора цепочки подполиэдров $K_{i}$ и согласуются с аксиомами Стинрода-Эйленберга. Можно показать, что так определенная группа $H_{\pi}^{m}(K, O ; M)$ совпадает с когомологиями Александера-Спеньера [23] и с пучковыми когомологиями [22], но нам это не понадобится.

Полиэдры $N_{i}$ и $U_{i}$. Рассмотрим отображение $f$ компактного полиэдра $X^{n}$ в ориентируемое кусочно линейное многообразие $Q^{m}$. В [7; лемма 2.1] была определена константа $\delta_{Q} \in(0,+\infty]$, связанная с размерами карт $Q$, или, что то же самое, с размерами окрестности $\Delta_{Q}$ в $Q \times Q$, отождествляемой с тотальным пространством касательного расслоения $Q$; в частности, $\delta_{\mathbb{R}^{m}}=+\infty$. Подбором метрики на $Q$ можно добиться, чтобы $\delta_{Q}$ было больше 1 , что мы и будем предполагать выполненньм в дальнейшем (если не заботиться о полноте метрики, можно, очевидно, добиться и $\delta_{Q}=1$, однако этого нам не потребуется). Рассмотрим теперь для произвольного $\varepsilon>0$ открытое подмножество $\Sigma_{f ; \varepsilon}=\{(x, y) \mid x \neq y$, $\operatorname{dist}(f(x), f(y))<\varepsilon\}$ взрезанного квадрата $\widetilde{X}$, которое можно также отождествить с прообразом $\varepsilon$-окрестности $\Delta_{Q}$ в $Q \times Q$ при ограничении $f^{2}: X \times X \rightarrow$ $Q \times Q$ на $\widetilde{X}$. Заметим, что семейство множеств $\Sigma_{f ; \varepsilon}$ есть фундаментальная система окрестностей “объединения $\Sigma_{f}$ и бесконечности”, т.е. $\Sigma_{f ; \varepsilon} \cup \Delta_{X}$ являются таковыми для $\Sigma_{f} \cup \Delta_{X}$. Пусть $T^{(d)}$ обозначает $d$-е барицентрическое измельчение какой-нибудь фиксированной эквивариантной триангуляции $T$ полиэдра $X \times X$ и $N_{d ; \varepsilon}=N\left(\Sigma_{f ; \varepsilon} \cup \Delta_{X}, T^{(d)}\right)$ - объединение всех (замкнутых) симплексов $T^{(d)}$, пеpeсекающих $\Sigma_{f ; \varepsilon} \cup \Delta_{X}$. Положим $N_{i}=N_{d ; 2^{-i}} \backslash \Delta_{X}$, где $d=d(i)$ - минимальное число такое, что $N_{i} \subset \Sigma_{2 \varepsilon}$. Таким образом, $N_{i}$ суть подполиэдры $\widetilde{X}$, образующие 
фундаментальную систему окрестностей “объединения $\Sigma_{f}$ и бесконечности”, причем для каждого $i$ выполнено $N_{i+1} \subset \Sigma_{f ; 2^{-i}} \subset \operatorname{Int} N_{i}$ и внешность $U_{i}=\overline{\widetilde{X} \backslash N_{i}}-$ компактный подполиэдр $\widetilde{X}$.

Препятствия $\vartheta(f)$ и $\check{\vartheta}(f)$. Пусть $f_{t}: X \rightarrow Q, t \in[0,1),-$ кусочно линейная гомотопия общего положения такая, что $f_{t} \rightarrow f$ равномерно при $t \rightarrow 1$. Будем рассматривать ее как отображение $F: X \times[0,1) \rightarrow Q \times[0,1)$. Напомним, что условие общего положения означает, что $F$ линейно на каждом симплексе некоторой триангуляции $T$ полиэдра $X \times[0,1)$ по отношению к некоторой комбинаторной триангуляции многообразия $Q \times[0,1)$, в которой симплициальны гомеоморфизмы $h_{\alpha}$ координатных карт на $\mathbb{R}^{m}$ (по отношению к некоторым триангуляциям $\mathbb{R}^{m}$ ), причем $F$-образы вершин $T$ максимально линейно независимы по отношению к каждому $h_{\alpha}$. Учитывая, что $F$ коммутирует с проекциями на $[0,1)$, потребуем дополнительно, чтобы триангуляция $T$ являлась разбиением (без добавления новых вершин) клеточной структуры, состояшей из произведений $\sigma \times I_{i}$ и $\sigma \times P_{i}$, где $I_{i}=\left[1-2^{-i}, 1-2^{-i-1}\right], P_{i}=\left\{1-2^{-i}\right\}$, а $\sigma$ - симплекс некоторой триангуляции $T_{i}$ полиэдра $X$, измельчающей $T_{i-1}$, для каждого $i \in \mathbb{N}$; без ограничения общности $F$ вкладывает каждую клетку. Найдется монотонно сходящаяся к нулю последовательность $\varepsilon_{i}>0$ такая, что конфигурационное сингулярное множество

$$
\Sigma_{F} \subset \widetilde{X} \times \Delta_{[0,1)} \subset \overline{X \times[0,1)}
$$

не пересекается с телескопом $U$ прямой последовательности полиэдров $U_{i}$ и включений $U_{i} \subset U_{i+1}$, вложенным в $\widetilde{X} \times \Delta_{[0,1)}$ так, что каждый цилиндр $U_{i} \times I$ отождествляется с $U_{i} \times\left[1-\varepsilon_{i}, 1-\varepsilon_{i+1}\right]$. Зафиксировав ориентации на $Q$, на $[0,1)$ и на каждом симплексе $T_{i}$ для каждого $i \in \mathbb{N}$, определим препятствие

$$
\vartheta(f) \in H_{\text {skew }}^{m}(\widetilde{X} \times[0,1), U) \simeq H_{\text {skew }}^{m}\left(\widetilde{X}, \widetilde{X} \backslash \Sigma_{f}\right)
$$

как класс коцикла, принимающего на клетке $\sigma \times \tau \times I_{i}$, где $\sigma, \tau$ - различные $k$-симплекс и $(m-k-1)$-симплекс $T_{i}$, значение, равное алгебраическому числу пересечений клеток $F\left(\sigma \times I_{i}\right)$ и $F\left(\tau \times I_{i}\right)$, а на клетке $\sigma \times \tau \times P_{i}$, где $\sigma, \tau-$ различные $k$-симплекс и $(m-k)$-симплекс $T_{i},-$ значение, равное алгебраическому числу пересечений клеток $F\left(\sigma \times P_{i}\right)$ и $F\left(\tau \times P_{i}\right)$.

Включение $\bigsqcup\left(\widetilde{X}, U_{i}\right) \subset(\widetilde{X} \times[0,1), U)$ индуцирует эпиморфизм

$$
H_{\text {skew }}^{m}\left(\widetilde{X}, \widetilde{X} \backslash \Sigma_{f}\right) \rightarrow \lim _{\longleftarrow} H_{\text {skew }}^{m}\left(\widetilde{X}, U_{i}\right) .
$$

Образ $\vartheta(f)$ при этом гомоморфизме есть нить ван Кампена-Скопенкова $\check{\vartheta}(f)$, состоящая из препятствий $\vartheta_{i}(f) \in H_{\mathrm{skew}}^{m}\left(\widetilde{X}, U_{i}\right)$ к $2^{-i}$-аппроксимируемости $f$ вложениями, которые были, по существу, определены в [19] (см. также [7]). Более инвариантное определение $\vartheta_{i}$ дано в лемме 3.3, (в), а выражение $\vartheta$ через собственный аналог $\vartheta_{i}-$ в наблюдении 3.2 .

ЗАмечАниЕ. В случае $Q=\mathbb{R}^{2 n}$ препятствие $\vartheta_{-\infty}\left(f^{2}\right) \in H_{\mathrm{eq}}^{m}(\widetilde{X})-$ в точности классическое препятствие ван Кампена к вложимости $X$ в $\mathbb{R}^{2 n}$ (см. [24]-[26]).

Теорема 1.2. Пусть $f: X^{n} \rightarrow Q^{2 n}$ - отображение компактного полиэдра в ориентируемое кусочно линейное многообразие, $n \geqslant 3$. Тогда

(a) [19], [7] $f$ дискретно реализуемо, если и только если $\breve{\vartheta}(f)=0$;

(б) [7], [5] $f$ изотопически реализуемо, если и только если $\vartheta(f)=0$.

Доказательство несложно (по модулю теоремы 1.1) и для полноты приводится ниже после необходимых пояснений по поводу применимости теории препятствий. 
КРИТЕРИЙ 1.3 [5]. Пусть $f: X^{n} \rightarrow Q^{2 n}$ - дискретно реализуемое отображсние компактного полиэдра в ориентируемое кусочно линейное многообразие, $n \geqslant 3$, причем для каждого $i=1,2, \ldots$ зафиксировано вложсение $g_{i}: X \hookrightarrow Q$, являющееся $2^{-i}-$ близким $\kappa f$. Тогда $f$ реализуемо изотопически в том и только том случае, когда в обратной последовательности, состоящей из групп $G_{i}=H_{\mathrm{eq}}^{2 n-1}\left(\widetilde{X}, U_{i}\right)$ и гомоморфизмов расширения носителя $\pi_{i}: G_{i+1} \rightarrow G_{i}$, разрешима бесконечная система уравнений

$$
x_{i}-\pi_{i} x_{i+1}=d_{\mathrm{eq}}\left(g_{i}, g_{i+1}\right) .
$$

Здесь $d_{\mathrm{eq}}(\varphi, \psi) \in H_{\mathrm{eq}}^{m-1}\left(\widetilde{X}, U_{i}\right)$-первоепрепятствие к изовариантной $2^{-i}$-гомотопии квадратов $\varphi^{2}, \psi^{2}: X \times X \rightarrow Q \times Q$ заданных $2^{-i}$-близких отображений $\varphi, \psi: X^{n} \rightarrow Q^{m}$, определяемое следующим образом. Пусть сначала $Q=\mathbb{R}^{m}$, тогда $d_{\text {eq }}(\varphi, \psi)$ есть просто первое препятствие $\mathrm{k} \mathbb{Z} / 2$-эквивариантной гомотопии $\operatorname{rel} U_{i}$ отображений моментов $\widetilde{\varphi}, \widetilde{\psi}: \widetilde{X} \rightarrow S^{m-1}[27 ; \S \S 2,4]$.

Пусть теперь $Q^{m}$ - произвольное ориентируемое PL-многообразие. В [7; лемма 2.1] показано, что изовариантная $2^{-i}$-гомотопность $2^{-i}$-близких изовариантных отображений $\Phi, \Psi: X \times X \rightarrow Q \times Q$, которые в силу их близости можно без ограничения обшности считать совпадающими на $U_{i}$, по сушеству равносильна эквивариантной 1-гомотопности $\operatorname{rel} U_{i}$ их ограничений на $\widetilde{X}$, где используется сделанное выше предположение о том, что $1<\delta_{Q}$. Чтобы сделать это утверждение строгим, нужно заменить $U_{i}$ на $U_{i+1}$ в части необходимости и на $U_{i-1}$ в части достаточности. Однако в дальнейшем мы не будем останавливаться на этом техническом моменте, подробно разъясненном в [7], оставляя уточнение индексов читателю. Принимая это во внимание и учитывая выполненную ввиду ориентируемости $Q$ тривиальность действия $\pi_{1}(Q)$ на $\pi_{m-1}\left(\widetilde{\mathbb{R}^{m}}\right)$, где $\mathbb{R}^{m}$ - карта многообразия $Q$, несложно повторить все построения стандартной $\mathbb{Z} / 2$-эквивариантной теории препятствий для отображений в сферу (см. выше), так что получится требуемое препятствие $d_{\mathrm{eq}}(\Phi, \Psi) \in H_{\mathrm{eq}}^{m-1}\left(\widetilde{X}, U_{i}\right)$.

Аналогично определяется первое препятствие $o_{\mathrm{eq}}(\Phi) \in H_{\mathrm{skew}}^{m}\left(\widetilde{X}, U_{i}\right)$ к продолжению частичного изовариантного отображения $\Phi^{\prime}: U_{i} \cup \Delta_{X} \rightarrow Q \times Q, 2^{-i}$-близкого к некоторому эквивариантному $\Phi: X \times X \rightarrow Q \times Q$, с сохранением изовариантности и близости к $\Phi$ (действительно, без ограничения общности $\Phi^{\prime}$ является ограничением $\Phi)$.

ДоКАЗАТЕЛЬСТво ТЕОРЕмЫ 1.2. Необходимость очевидна. Остановимся на достаточности. Несложно видеть, что $\vartheta_{i}(f)$ совпадает с первым препятствием $o_{\text {eq }}\left(f^{2}\right) \in H_{\text {skew }}^{m}\left(\widetilde{X}, U_{i}\right)$, которое в данных размерностях является полным, а $\vartheta(f)$ - с первым препятствием $o_{\text {eq }}\left(\left.F^{2}\right|_{X \times X \times[0,1)}\right) \in H_{\text {skew }}^{m}(\widetilde{X} \times[0,1), U)$ к продолжению с сохранением $\varepsilon$-близости к $\left.F^{2}\right|_{X \times X \times[0,1)}$, где $\varepsilon: X \times X \times[0,1) \rightarrow(0,1]-$ подходящая собственная функция. Несмотря на то что во втором случае наше препятствие не является полньм по размерностньм соображениям, его тривиальность доставляет отображение $\Phi: X \times X \times[0,1) \rightarrow Q \times Q$ такое, что $\Phi^{-1}\left(\Delta_{Q}\right) \backslash \Delta_{X}$ содержится во внутренности симплексов максимальной размерности подходящей триангуляции. Эти сингулярности устраняются рассуждениями, аналогичными использованным при доказательстве [5; добавление 1.2$]$ и [4; следствие $1.8 \mathrm{a}]$. 
ДОКАЗАТЕЛЬСТВО КРИТЕРИЯ 1.3. Если $f$ изотопически реализуемо, имеется последовательность вложений $f_{i}: X \hookrightarrow Q$ такая, что $f_{i}$ является $2^{-i}$-близким к $f$, причем $f_{i}$ и $f_{i+1}$ соединяются $2^{-i}$-изотопией, в частности, $d_{\mathrm{eq}}\left(f_{i}, f_{i+1}\right)=0$. Но тогда $x_{i}=d_{\mathrm{eq}}\left(g_{i}, f_{i}\right)$ доставляют искомое решение.

Обратно, предположим, что система (1) разрешима. Тогда для классов $d_{i}^{m} \in$ $H_{\text {skew }}^{m}\left(\widetilde{X} \times I, \widetilde{X} \times \partial I \cup U_{i} \times I\right)$, соответствуюших первьм препятствиям $d_{\mathrm{eq}}\left(g_{i}, g_{i+1}\right)$ при изоморфизме Тома, выполнено $d_{i}^{m}=\delta^{*}\left(p_{i}^{*}\left(x_{i}\right)-q_{i}^{*}\left(\pi_{i} x_{i+1}\right)\right)$ для каждого $i$, где $\delta^{*}$ - связываюший гомоморфизм из точной последовательности тройки $(\widetilde{X} \times I$, $\left.\widetilde{X} \times \partial I \cup U_{i} \times I, U_{i} \times I\right)$, а отображения $p_{i}, q_{i}:\left(\widetilde{X}, U_{i}\right) \times \partial I \rightarrow\left(\widetilde{X}, U_{i}\right)$ переводят одно из дизъюнктных слагаемых в точку, а второе гомеоморфно. Просуммировав эти равенства по $i$, получим, что класс

$$
\sum_{i=0}^{\infty} d_{i}^{m} \in H_{\mathrm{eq}}^{m}(\widetilde{X} \times[0,1), \widetilde{X} \times N \cup U),
$$

где $N=\left\{1-2^{-i} \mid i=0,1,2, \ldots\right\}$, лежит в образе связываюшего гомоморфизма из точной последовательности тройки $(\widetilde{X} \times[0,1), \widetilde{X} \times N \cup U, U)$. Но легко видеть, что образ $\sum d_{i}^{m}$ в группе $H_{\mathrm{eq}}^{m}(\widetilde{X} \times[0,1), U)$ есть не что иное, как $\vartheta(f)$.

ЗАмЕчАниЕ. Если $\left(x_{i}\right)$ - решение системы $(1)$, то любое другое решение получается прибавлением некоторой нити, т.е. решения однородной системы. Отметим, что в системе (1), вообще говоря, нельзя вместо минусов писать плюсы. Действительно, делая в "плюсовой" системе $x_{i}+\pi x_{i+1}=a_{i}$ замену переменных $y_{i}=(-1)^{i} x_{i}$, получаем "минусовую" $y_{i}-\pi y_{i+1}=(-1)^{i} a_{i}$. Остается заметить, что в обратной последовательности из умножений на 3 в группе $\mathbb{Z}$ система $x_{i}-3 x_{i+1}=2$ разрешима (достаточно взять все $x_{i}$ равными -1 ), а система $x_{i}-3 x_{i+1}=(-1)^{i} \cdot 2-$ нет, поскольку после вычитания из нее очевидно разрешимой системы $x_{i}-3 x_{i+1}=k_{i}$, где $k_{2 j}=1, k_{2 j+1}=-3$, она преврашается в систему $x_{i}-3 x_{i+1}=1$, которая, как известно, неразрешима (см. [4; пример 1.9], [7; пример во введении], [5; пример 4]).

Функтор производного предела. Напомним (подробнее см. [23], [28]-[30]), что (обратный) предел и производный (функтор от функтора обратный) предел обратной последовательности абелевых групп $\left\{G_{i} ; \pi_{i}\right\}$ определяются как ядро и коядро отображения $f: \prod G_{i} \rightarrow \prod G_{i}$, переводящего элемент $\left(a_{1}, a_{2}, \ldots\right)$ в элемент $\left(a_{1}-\pi a_{2}, a_{2}-\pi a_{3}, \ldots\right)$ :

$$
0 \rightarrow \lim _{\longleftarrow}\left\{G_{i} ; \pi_{i}\right\} \rightarrow \prod_{i} G_{i} \stackrel{f}{\longrightarrow} \prod_{i} G_{i} \rightarrow \lim ^{1}\left\{G_{i} ; \pi_{i}\right\} \rightarrow 0 .
$$

Таким образом, система (1) разрешима, если и только если задаваемый ее коэффициентами элемент производного предела $\lim ^{1}\left\{G_{i} ; \pi_{i}\right\}$ тривиален. Несложно видеть, что этот элемент не зависит от выбора вложений $g_{i}$ (например, это ясно из нижеследуюшего). Высшие производные функторы обратного предела нетривиальны для некоторых обратных спектров счетных абелевых групп, индексированных не вполне упорядоченными множествами (см. [31]). Для обратной последовательности счетных абелевых групп тривиальность производного предела равносильна условию Миттаг-Леффлера: для каждого натурального $i$ образы групा $G_{j}, j \geqslant i$, в группе $G_{i}$ стабилизируются, начиная с некоторого $j=j_{0}(i)$ [32] (см. также [33]). 
Нам понадобится известный факт о том, что производный предел обратной последовательности из конечных групп тривиален (поскольку в ней выполнено условие Миттаг-Леффлера; альтернативное доказательство указано в лемме 5.1,(б)).

Заметим, что в предположении дискретной реализуемости $f$ класс системы (1) в производном пределе переходит в $\vartheta(f)$ при гомоморфизме из короткой точной последовательности типа Милнора [23], [33]

$$
0 \rightarrow \lim ^{1} H_{\mathrm{eq}}^{m-1}\left(\widetilde{X}, U_{i}\right) \rightarrow H_{\text {skew }}^{m}\left(\widetilde{X}, \widetilde{X} \backslash \Sigma_{f}\right) \rightarrow \lim _{\longleftarrow} H_{\text {skew }}^{m}\left(\widetilde{X}, U_{i}\right) \rightarrow 0,
$$

что проясняет связь критерия 1.3 и следствия 1.2. Это утверждение по существу содержится в вышеприведенном доказательстве критерия 1.3; построение таких последовательностей напоминается в [5; приложение] на примере аналогичной последовательности в гомологиях (причем если $X$ - многообразие, эти две последовательности связаны двойственностью Александера).

\section{§2. Отображения в гиперплоскость}

Дадим в качестве иллюстрации критерия 1.3 новое доказательство теоремы о стабилизации с коразмерностью 1 в простейшем случае.

Tеорема 2.1 [7], [5; добавление 1.2]. Пусть $X^{n}$ - компактный полиәдр, $Q^{m-1}$ - ориентируемое PL-многообразие, $m \geqslant 3(n+1) / 2$. Eсли $f: X \rightarrow Q$ дискретно реализуемо, то композичия $f$ и включения $Q \subset Q \times \mathbb{R}$ изотопически реализуема.

ДокАЗАТЕЛЬСТво ДЛЯ СЛУЧАЯ $Q=\mathbb{R}^{2 n-1}$. Пусть $\left(g_{i}\right)$ - последовательность вложений как в критерии 1.3 , т.е. $\forall i$ вложение $g_{i}: X \hookrightarrow \mathbb{R}^{2 n}$ является $2^{-i}$-близким к $f$. Рассмотрим разность

$$
d_{i}=\left(\widetilde{g}_{i}\right)_{\mathrm{eq}}^{*}\left(\xi_{\mathrm{eq}}\right)-\left(\widetilde{g}_{i+1}\right)_{\mathrm{eq}}^{*}\left(\xi_{\mathrm{eq}}\right)
$$

коциклов, индуцированњых отображениями моментов из некоторой образующей $\xi_{\text {eq }} \in Z_{\text {eq }}^{2 n-1}\left(S^{2 n-1}\right)=\mathbb{Z}$. Условимся здесь для определенности, что на сфеpe $S^{2 n-1}$ зафиксирована стандартная $\mathfrak{s - э к в и в а р и а н т н а я ~ с т р у к т у р а ~ C W - к о м п л е к с а , ~}$ содержащего по паре клеток в каждой размерности. Если $\widetilde{g}_{i}$ и $\widetilde{g}_{i+1}$ клеточные и совпадают на $(2 n-2)$-остове, то нетрудно видеть (см. доказательство леммы 2.3 ниже), что разность $d_{i}$ равна (уже в группе коцепей) удвоенной различающей $2 d_{\mathrm{eq}}^{2 n-1}\left(\widetilde{g}_{i}, \widetilde{g}_{i+1}\right)$.

$\mathrm{C}$ другой стороны, в группе когомологий как различающая, так и разность индуцированных коциклов имеет инвариантньй смысл, не зависящий от разбиения на клетки и не меняющийся при эквивариантной гомотопии. Но если образ каждого $g_{i}$ лежит в гиперплоскости $\mathbb{R}^{2 n-1}$, то и каждая разность $d_{i}$ равна нулю. Заметим, наконец, что ввиду $2^{-i}$-близости вложений $g_{i}$ и $g_{i+1}$ к отображению $f$ эквивариантную гомотопию, совмешающую отображения $\widetilde{g}_{i}$ и $\widetilde{g}_{i+1}$ на $(2 n-2)$-остове, можно произвести, не выходя из гиперплоскости $\mathbb{R}^{2 n-1}$ на подполиэдре $U_{i}$. Таким образом, корректно определен относительный класс когомологий

$$
\left[d_{i}\right] \in H_{\mathrm{eq}}^{2 n-1}\left(\widetilde{X}, U_{i}\right)=G_{i}
$$

являющийся разностью $\left(\widetilde{g}_{i}\right)_{\mathrm{eq}}^{*}\left(\left[\xi_{\text {eq }}\right]\right)-\left(\widetilde{g}_{i+1}\right)_{\mathrm{eq}}^{*}\left(\left[\xi_{\mathrm{eq}}\right]\right)$ обратных образов образующей $\left[\xi_{\mathrm{eq}}\right] \in H_{\mathrm{eq}}^{2 n-1}\left(S^{2 n-1}, S^{2 n-2}\right)$ и равный, с одной стороны, классу когомологий 
$2 d_{\mathrm{eq}}\left(\widetilde{g}_{i}, \widetilde{g}_{i+1}\right) \in G_{i}$ удвоенной различающей $2 d_{\mathrm{eq}}^{2 n-1}\left(\widetilde{g}_{i}, \widetilde{g}_{i+1}\right)$, а с другой стороны - нулю.

Значит, первые препятствия $d_{\text {eq }}\left(\widetilde{g}_{i}, \widetilde{g}_{i+1}\right) \in G_{i}$ суть элементы порядка 2 . Но тогда система (1) разрешима, так как ее правые части лежат в обратной последовательности из конечных групा (а именно 2-кручений $G_{i}$ ).

Предположим теперь, что образ отображения $f: X \rightarrow \mathbb{R}^{2 n}$ по-прежнему лежит в гиперплоскости $\mathbb{R}^{2 n-1}$, но известно лишш, что $f$ дискретно реализуемо как отображение в $\mathbb{R}^{2 n}$.

Посмотрим вначале, что получится, если действовать, как в приведенном выше частичном доказательстве теоремы 2.1. Условие $f(X) \subset \mathbb{R}^{2 n}$ можно использовать следующим образом: если вложение $g_{i}: X \hookrightarrow \mathbb{R}^{2 n}$ является $2^{-i}$-близким к $f$, то коцикл $z_{i}=\left(\widetilde{g}_{i}\right)_{\mathrm{eq}}^{*}\left(\xi_{\mathrm{eq}}\right)$, индуцированный из образующей $\xi_{\mathrm{eq}} \in Z_{\mathrm{eq}}^{2 n-1}\left(S^{2 n-1}\right)=\mathbb{Z}$, имеет носитель вне $U_{i}$. При $l \leqslant i$ обозначим через $\left[z_{i}\right]_{l}$ класс коцикла $z_{i}$ в группе $G_{l}$ и заметим снова, что

$$
\left[z_{i}\right]_{i}-\left[z_{i+1}\right]_{i}=2 d_{\mathrm{eq}}\left(\widetilde{g}_{i}, \widetilde{g}_{i+1}\right) .
$$

Поэтому, если каждый элемент $\left[z_{i}\right]_{i}$ делится на 2, можно сделать замену переменных $y_{i}=x_{i}-\left[z_{i}\right]_{i} / 2$, получив новую систему

$$
y_{i}-p y_{i+1}=d_{\mathrm{eq}}\left(\widetilde{g}_{i}, \widetilde{g}_{i+1}\right)-\frac{\left[z_{i}\right]_{i}-\left[z_{i+1}\right]_{i}}{2},
$$

разрешимость которой эквивалентна разрешимости исходной системы (1). Но новая система разрешима, поскольку ее правые части лежат в 2-кручениях. Доказан случай $Q=\mathbb{R}^{2 n-1}$ следующего предложения.

ПРЕДЛОЖЕНИЕ 2.2. Пусть $X^{n}$ - компактнъй полиәдр, $Q^{m-1}$ - ориентируемое PL-многообразие и отображение $f: X \rightarrow Q \subset Q \times \mathbb{R}$ дискретно реализуемо. Препятствие $(f)$ к изотопической реализуемости - элемент порядка 2.

Доказательство в общем случае аналогично с учетом п. (а) леммы 2.3.

ЗАмЕчАниЕ. Приведенные выше рассуждения не проходят, если не все $\left[z_{i}\right]_{i}$ делятся на 2. Тем не менее, из них ясно, что для нахождения достаточных условий разрешимости исходной системы (в терминах свойств компакта $\Sigma_{f}$ ) можно было бы исследовать вопрос о поднятии решения системы с удвоенными коэффициентами в правой части, т.е. о поднятии относительно гомоморфизма умножения на 2: $G_{i} \rightarrow G_{i}, i=0,1,2, \ldots$ Оказьвается, что этот гомоморфизм можно разложить в композицию двух и поднятие достаточно осуществить лишш относительно одного из них; это соображение является исходньм пунктом теории, построенной в $\S \S 4-6$.

Лемма 2.3. В условиях предложения 2.2 пусть $g, h: X \hookrightarrow Q \times \mathbb{R}$ - вложения, $2^{-i}-$ близкие $\kappa f$. Тогда

(a) (cp. [7; замечание к теореме 1]) $2 d_{\mathrm{eq}}(g, h)=\left(g^{2}\right)_{\mathrm{eq}}^{*}\left(\xi_{\mathrm{eq}}\right)-\left(h^{2}\right)_{\mathrm{eq}}^{*}\left(\xi_{\mathrm{eq}}\right)$, әде $\xi_{\mathrm{eq}} \in H_{\mathrm{eq}}^{m-1}(\widetilde{Q \times \mathbb{R}}, \widetilde{Q})-$ класс когомологий, двойственный $\kappa\left[\Delta_{Q} \times \widetilde{\mathbb{R}}\right]$;

(б) $d(g, h)=\left(g^{2}\right)^{*}(\xi)-\left(h^{2}\right)^{*}(\xi)$, где $\xi \in H^{m-1}(\widetilde{Q \times \mathbb{R}}, \widetilde{Q})-$ класс когомолоәий, двойственный $\kappa\left[\Delta_{Q} \times R\right]$, а $R$ обозначает любую связную компоненту $\widetilde{\mathbb{R}}$.

Корректность взятия обратных образов $\xi_{\text {eq }}$ и ясна из вышеприведенного частичного доказательства теоремы 2.1. 
ДокАЗАТЕЛЬСтво. Если $\Phi, \Psi: X^{2} \rightarrow(Q \times \mathbb{R})^{2}$ - изовариантные отображения, $2^{-i}$-близкие к $f^{2}$ и совпадающие на $(m-2)$-остове, первое препятствие к гомотопии $d(\Phi, \Psi) \in H^{m-1}\left(\widetilde{X}, U_{i}\right)$ принимает на каждом $(m-1)$-симплексе $\sigma$ значение, отвечаюшее сфероиду $\chi: S^{m-1} \rightarrow \widetilde{\mathbb{R}^{m}}$, заданному отображением $\left.\Phi\right|_{\sigma}$ на северном полушарии и $\left.\Psi\right|_{\sigma}$ на южном, где $\left(\mathbb{R}^{m}, \mathbb{R}^{m-1}\right)$ отождествлено с соответствуюшей локальной картой $(Q \times \mathbb{R}, Q)$. Первое препятствие $d_{\mathrm{eq}}(\Phi, \Psi) \in H_{\mathrm{eq}}^{m-1}\left(\widetilde{X}, U_{i}\right)$ к эквивариантной гомотопии совпадает с $d(\Phi, \Psi)$ как коцикл, однако рассматривается с точностью до кограниц симметричных, а не произвольных коциклов. Значение $d(\Phi, \Psi)$ на $\sigma$ равно индексу пересечения отображения $\chi$ с замкнутьм подмногообразием $\Delta_{\mathbb{R}^{m-1}} \times R$, т.е. целому числу $\Phi^{*}(n)-\Psi^{*}(n)$ в группе $H^{m-1}(\sigma, \partial \sigma)$, отождествленной с $\mathbb{Z}$ выбором ориентации на $\sigma$, где $n \in H^{m-1}\left(\widetilde{\mathbb{R}^{m}}, \widetilde{\mathbb{R}^{m-1}}\right)$ - класс когомологий, двойственный к $\left[\Delta_{\mathbb{R}^{m-1}} \times R\right]$. Следовательно, $d(\Phi, \Psi)=\Phi^{*}(\xi)-\Psi^{*}(\xi)$, причем ввиду инвариантности обеих сторон равенства при гомотопии оно остается справедливым и без предположения о совпадении $\Phi$ и $\Psi$ на $(m-2)$-остове, в частности, $d(g, h)=\left(g^{2}\right)^{*}(\xi)-\left(h^{2}\right)^{*}(\xi)$. С другой стороны, ввиду произвольности выбора связной компоненты $R$ вьполнено $2 d(\Phi, \Psi)=\Phi^{*}\left(\xi+\mathfrak{t}^{*} \xi\right)-\Psi^{*}\left(\xi+\mathfrak{t}^{*} \xi\right)$, откуда $d_{\mathrm{eq}}(\Phi, \Psi)=\Phi_{\mathrm{eq}}^{*}\left(\xi_{\mathrm{eq}}\right)-\Psi_{\mathrm{eq}}^{*}\left(\xi_{\mathrm{eq}}\right)$, где обе стороны инвариантны при эквивариантной гомотопии, так что совпадение $\Phi$ и $\Psi$ на $(m-2)$-остове снова необязательно, и мы получаем формулу из (а).

Примеры. Будем строить отображение $S^{n} \rightarrow \mathbb{R}^{2 n-1} \subset \mathbb{R}^{2 n}$, реализуемое дискретно, но не изотопически, отправляясь от известного примера отображения $\alpha: S^{n} \rightarrow \mathbb{R}^{2 n-1} \subset \mathbb{R}^{2 n}, n=2 k+1 \geqslant 3$, не реализуемого дискретно. Сингулярное множество $\alpha$ нетривиально двулистно накрывает (посредством $\alpha$ ) кривую его двойных точек, а именно окружность, причем локально последняя устроена как трансверсальное пересечение двух листов (подробности приведены, например, в $[34 ;$ гл. $1, \S 1])$, так что при полном (однократном) ее обходе листы меняются местами. (Заметим, что ориентируемость сферы $S^{n}$ приводит к требованию сохранения ориентации центральной симметрией в $\mathbb{R}^{n-1}$, по которой лист склеивается с собой при двукратном обходе, откуда и возникает условие нечетности $n$.) Зная примеры отображений, реализуемых дискретно, но не изотопически, естественно попытаться модифицировать конструкцию $\alpha$, заменив трансверсальное пересечение "фальшивым", локально устроенньм как произведение такого примера на отрезок. Однако здесь мы сталкиваемся с некоторой трудностью, поскольку все найденные ранее примеры дискретно, но не изотопически реализуемых отображений в коразмерности $\geqslant 3[4]$, [7], [5] обладали сушественной асимметрией, не позволяющей разумно интерпретировать перестановку двух “фальшивых” листов. Пробел восполняет следующий “симметричный”

ПримеР 1. Приведем новый пример отображения в коразмерности три, реализуемого дискретно, но не изотопически (первьй такой пример был построен в [4] и усовершенствован в [7]) и вдобавок являюшегося локально плоским топологическим погружением. Именно, для любого $n \geqslant 3$ строится отображение $S^{n} \rightarrow \mathbb{R}^{2 n}$ с описанными свойствами; для наглядности проведем построение, считая, что $n=3$.

Заметим предварительно, что в сфере $S^{3}$ будут взяты два непересекающихся полнотория и отправлены в $\mathbb{R}^{6}$ так, что пара 3 -адических соленоидов, содержащихся в этих полноториях, перейдет в один соленоид, а на дополнениях к соленоидам отображение будет вложением, причем двумерные поперечные срезы полно- 
ториев будут зацепляться друг с другом бесконечной внутренней связной суммой $1+3+9+\cdots$ стандартных хопфовских зацеплений $S^{2} \sqcup S^{2} \rightarrow \mathbb{R}^{5}$, накапливающихся к канторову множеству, по которому $\mathbb{R}^{5}$ срезает соленоид. Перейдем теперь к подробному построению.

Представим сферу $S^{5}$ как джойн $S_{0}^{2} * S_{1}^{2}$ двух сфер, зацепленных в ней с коэфрициентом 1. Диагональное вложение $S^{2} \hookrightarrow S^{2} \times S^{2}$ доставляет стандартное включение произведения $S^{2} \times I$ в этот джойн, так что $S^{2} \times i$ отождествляется с $S_{i}^{2}$, где $i=0,1$. Зафиксируем четыре произвольные попарно различные точки $x_{0}, x_{1}, x_{2}, x_{3} \in S^{2}$, их дисковые окрестности $D_{j}^{2}$ в сфере $S^{2}$ и шаровые окрестности $B_{j}^{5}$ точек $x_{j} \times \frac{1}{2}$ в сфиере $S^{5}, j=0, \ldots, 3$, определенные как $D_{j}^{2} \times D_{j}^{2} \times\left[\frac{1}{4}, \frac{3}{4}\right]$ (мы воспользовались представлением $S^{5} \backslash\left(S_{0}^{2} \cup S_{1}^{2}\right)$ в виде произведения $S_{0}^{2} \times S_{1}^{2} \times(0,1)$, доставляемьм структурой джойна).

Выкинем внутренности дисков $D_{j}^{2}$ из сферы $S^{2}$ и внутренности шаров $B_{j}^{5}$ из сферы $S^{5}$ и обозначим полученные многообразия с краем через $P$ и $Q$ соответственно. Определим вложение $g_{i}:(P, \partial P) \hookrightarrow(Q, \partial Q)$, где $i=0,1$, добавив воротник к многообразию $P$, т.е. зафиксировав гомеоморфизм $P \cong P \cup_{\partial P} \partial P \times I$, после чего рассмотрев включение $P \subset S^{2} \cong S_{i}^{2}$ и переведя воротник $\partial P \times I$ на четыре трубки $\partial P \times J$, где $J$ обозначает $\left[0, \frac{1}{4}\right]$ в случае $i=0$ и $\left[\frac{3}{4}, 1\right]$ в случае $i=1$. Обозначим также $\overline{S^{2} \backslash D_{0}^{2}}$ и $\overline{S^{5} \backslash B_{0}^{5}}$ через $D^{2}$ и $B^{5}$ соответственно и определим аналогичным образом вложение $g_{i}^{\prime}:\left(D^{2}, \partial\right) \hookrightarrow\left(B^{5}, \partial\right)$. Определим еще для каждого $j$ сферу $S_{j}^{3} \subset \partial B_{j}^{5}$, содержащую обе окружности $g_{0}\left(\partial D_{j}^{2}\right)$ и $g_{1}\left(\partial D_{j}^{2}\right)$, как образ сферы $\partial D_{0, j}^{2} * \partial D_{1, j}^{2}$, где $D_{i j}^{2}=D_{j}^{2} \subset S^{2} \cong S_{i}^{2}$, при каком-нибудь гомеоморфизме $\rho_{j}$ сферы $S^{5}$, переводящем $D_{0, j}^{2} * D_{1, j}^{2}$ на $B_{j}^{5}$, так чтобы $D_{i j}^{2}$ переходило в $D_{0, j}^{2} \times D_{1, j}^{2} \times\left\{\frac{1}{4}+\frac{i}{2}\right\}$, и эквивариантном относительно инволюции $\sigma$, переставляющей сомножители джойна $S_{0}^{2} * S_{1}^{2}$.

Рассматривая по очереди каждую сферу $\partial B_{j}^{5}$, где $j=1,2,3$, как экватор сферы $S^{5}$, определим автогомеоморфизм $\theta_{j}$ сферы $S^{5}$ как “поворот на $\pi^{\prime \prime 2}$, переводящий северное полушарие в южное и неподвижньй на $S_{j}^{3}$, точнее, зададим $\theta_{j}$ как $\widehat{\theta}_{j}^{\rho_{j}}$, где $\widehat{\theta}_{j}$ - джойн тождества на $\partial D_{0, j}^{2} * \partial D_{1, j}^{2}$ и переставляющей сомножители инволюции на $S_{0, j}^{0} * S_{1, j}^{0}$, где $S_{i j}^{0} * \partial D_{i j}^{2}=S_{i}^{2}$. Легко видеть, что $\theta_{j}$ эквивариантен относительно $\sigma$. Считая экватором по очереди каждую сферу $\theta_{j}\left(\partial B_{k}^{5}\right), k \neq j$, получим еще девять автогомеоморфизмов $\theta_{j k}$. Проитерируем этот процесс очевидньм образом и определим автогомеоморфизм $\psi_{i_{1}} i_{2} \ldots i_{k}$, где $k$ - целое неотрицательное и $\forall j<k$ индекс $i_{j}$ принимает одно из значений $1,2,3$, как композицию

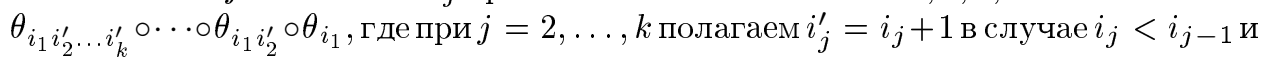
$i_{j}^{\prime}=i_{j}$ в случае $i_{j}>i_{j-1}$. Тогда образы $Q$ при различных гомеоморфизмах $\psi_{*}$ заполнят всю сферу $S^{5}$ за исключением внутренности шара $B_{0}^{5}$ и канторова множества $\mathscr{D}$. Аналогичным образом определяется серия автогомеоморфизмов $\varphi_{i_{1}} i_{2} \ldots i_{k}$ сфферы $S^{2}$. Образы $P$ при различных гомеоморфизмах $\varphi_{*}$ заполнят всю сферу $S^{2}$ за исключением внутренности диска $D_{0}^{2}$ и канторова множества $\mathscr{C}$. При $i=0,1$ продолжим вложение $g_{i}$ до вложения $\bar{g}_{i}:\left(D^{2}, \partial\right) \hookrightarrow\left(B^{5}, \partial\right)$, используя композиции

\footnotetext{
2 Зеркальная симметрия относительно экваториальной сферы нас не устраивает, так как она изменяет ориентацию и тем самым коэффициенты зацепления двумерных сфер, а "гомотетия" с центром во внутренности $B_{j}^{5}$ и с бесконечностью во внутренности какого-нибудь $B_{k}^{5}$, $k \neq j$, неудобна тем, что не является неподвижной на $g_{i}\left(\partial D_{j}^{2}\right), i=0,1$.
} 
$\psi_{*} \circ g_{i} \circ \varphi_{*}^{-1}$ и продолжая по непрерывности, так что $\bar{g}_{i}(\mathscr{C})=\mathscr{D}$ (здесь используется, что эти композиции согласованы друг с другом и исходным вложением $\varphi$ ввиду поточечной инвариантности сфер $S_{j}^{3}$ при гомеоморфизмах $\theta_{j}$ ). Объединение $\bar{g}=g_{1} \sqcup g_{2}:\left(D^{2} \sqcup D^{2}, \partial\right) \rightarrow\left(B^{5}, \partial\right)$, таким образом, не является вложением, однако аппроксимируется вложениями $\bar{g}_{k}^{\prime}$, полученными с использованием композиций $\psi_{i_{1} i_{2} \ldots i_{k}} \circ g_{i}^{\prime} \circ \varphi_{i_{1} i_{2} \ldots i_{k}}^{-1}$.

Пусть $h_{i j}$ - какой-нибудь сохраняющий ориентацию автогомеоморфизм $S^{2}$, неподвижный на $D_{k}^{2}$ при $k \neq i, j$ и переставляющий шары $B_{i}^{2}, B_{j}^{2}$. Тогда $H_{i j}=$ $h_{i j} * h_{i j}$ - автогомеоморфизм $S^{5}$, согласованный со структурой джойна $S_{0}^{2} * S_{1}^{2}$, неподвижный на $B_{k}^{5}$ при $k \neq i, j$ и переставляющий шары $B_{i}^{5}, B_{j}^{5}$. Обозначим $T=H_{12} \circ H_{23}$ и $K_{k}=\psi_{\underbrace{11 \ldots 1}_{k \text { раз }}} \circ H_{01}$. Тогда бесконечная композиция

$$
\cdots \circ\left(K_{2} \circ T \circ K_{2}^{-1}\right) \circ\left(K_{1} \circ T \circ K_{1}^{-1}\right) \circ T
$$

есть корректно определенньй автогомеоморфизм $H$ сферы $S^{5}$ такой, что для каждого допустимого набора $i_{1}, \ldots, i_{k}$ его $3^{k}$-я итерация $H^{3^{k}}$ циклически переставляет шары $\psi_{i_{1} i_{2} \ldots i_{k}}\left(B_{1}^{5}\right), \psi_{i_{1} i_{2} \ldots i_{k}}\left(B_{2}^{5}\right)$ и $\psi_{i_{1} i_{2} \ldots i_{k}}\left(B_{3}^{5}\right)$. Поскольку каждый $H_{i j}$ согласован со структурой джойна, он индуцирует $h_{i j}$ на $S^{2}$ с помощью любого из вложений $g_{i}$ (в предположении, что $h_{i j}$ индуцирует стандартные гомеоморфизмы на воротниках шаров $D_{k}^{2}$ ). Следовательно, $H$ индуцирует с помощью любого из вложений $\bar{g}_{i}$ автогомеоморфизм $h$ на $S^{2}$, определенный аналогично $H$ с заменой $H_{i j}$ на $h_{i j}$ и $\psi_{*}$ на $\varphi_{*}$. Поскольку автогомеоморфизмы $\left.H\right|_{B^{5}}$ и $\left.h\right|_{D^{2}}$ неподвижны на границе, они изотопны тождеству и, значит, их торы гомеоморфны $B^{5} \times S^{1}$ и $D^{2} \times S^{1}$ соответственно. Легко видеть, однако, что торы $\mathscr{C}^{\prime}$ и $\mathscr{D}^{\prime}$ их ограничений на $\mathscr{C}$ и $\mathscr{D}$ гомеоморфны 3 -адическим соленоидам. Пусть

$$
G:\left(D^{2} \times S^{1} \sqcup D^{2} \times S^{1}, \partial\right) \rightarrow\left(B^{5} \times S^{1}, \partial\right)
$$

определяется как тор действия этих автогомеоморфизмов на $\bar{g}$. Отображение $G$ переводит каждый экземпляр $\mathscr{C}^{\prime}$ гомеоморфно на $\mathscr{D}^{\prime}$ и является вложением на дополнении к этим двум экземплярам.

На границе имеем вложение $\left.G\right|_{\partial}: S^{1} \times S^{1} \sqcup S^{1} \times S^{1} \hookrightarrow S^{4} \times S^{1}$, разлагающееся в произведение некоторого $S^{1} \sqcup S^{1} \hookrightarrow S^{4}$ и тождества $S^{1} \rightarrow S^{1}$. Следовательно, $\left.G\right|_{\partial}$ продолжается до вложения $G^{\prime}:\left(S^{1} \times D^{2} \sqcup S^{1} \times D^{2}, \partial\right) \hookrightarrow\left(S^{4} \times D^{2}, \partial\right)$. Объединение $G \cup_{\partial} G^{\prime}: S^{3} \sqcup S^{3} \rightarrow S^{6}$ с помощью связной суммы и прокальвания преврашается окончательно в $\bar{G}: S^{3} \rightarrow \mathbb{R}^{6}$. Совершенно аналогичные построения доставляют серию отображений $\bar{G}_{n}: S^{n} \rightarrow \mathbb{R}^{2 n}$ для всех $n \geqslant 3$. Очевидно, $\bar{G}_{n}$ дискретно реализуемо и является локально плоским топологическим погружением. Изотопическая нереализуемость проверяется непосредственно с помощью очевидной необходимой части критерия 1.3 (см. замечание ниже) - аналогично проверке в $[4 ;$ пример 1.9$]$ - либо с помощью очевидной необходимой части теоремы $3,(\mathrm{a})-$ аналогично [7; проверка нетривиальности препятствия $\left.o^{!}\left(\mu_{p}^{n}\right)\right]$.

ПримеР 2. В этом и следуюшем примерах мы строим локально плоское топологическое погружение $S^{n} \rightarrow \mathbb{R}^{2 n-1} \subset \mathbb{R}^{2 n}$, реализуемое дискретно, но не изотопически, при условиях $n=2 k \geqslant 4$ и $n=2 k+1 \geqslant 5$ соответственно. Рассмотрим отображение

$$
G_{n}:\left(D^{n-2} \times S^{1} \sqcup D^{n-2} \times S^{1}, \partial\right) \rightarrow\left(B^{2 n-3} \times S^{1}, \partial\right), \quad n-1 \geqslant 3,
$$


построенное выше в случае $n-1=3$. Из построения ясно, что инволюция $\mathfrak{s}_{n}$, меняюшая местами два экземпляра $D^{n-2} \times S^{1}$, реализуется посредством $G_{n}$ и инволюции $\mathfrak{t}_{n}:=\sigma \times \mathrm{id}_{S^{1}}$ на $B^{2 n-3} \times S^{1}$, сохраняющей ориентацию в случае нечетности $n .{ }^{3}$ Казалось бы, отсюда получается искомое отображение при нечетном $n$, а именно очевидное отображение между торами этих инволюций, продолженное некоторьм образом до отображения $S^{n} \rightarrow \mathbb{R}^{2 n-1}$ и взятое в композиции со включением в $\mathbb{R}^{2 n}$. Однако сразу можно заметить, что оно дискретно реализуемо уже в гиперплоскости и поэтому согласно теореме 2.1 не может не допускать изотопической реализации в $\mathbb{R}^{2 n}$.

Вернемся на время к рассмотрению отображения $\bar{g}:\left(D^{2} \sqcup D^{2}, \partial\right) \rightarrow\left(B^{5}, \partial\right)$ из построения примера 1 , которое, напомним, было получено из вложений

$$
g_{0}, g_{1}:(P, \partial P) \hookrightarrow(Q, \partial Q),
$$

размноженных с помощью гомеоморфизмов $\varphi_{*}$ и $\psi_{*}$. Пусть $u$ - какой-нибудь обращающий ориентацию гомеоморфизм $Q \rightarrow Q$; по общему положению его можно считать неподвижньм на $g_{i}$-образах четырех окружностей $\partial P$. Вложениям $g_{0} \sqcup g_{1}$ и $u \circ\left(g_{0} \sqcup g_{1}\right)$ можно приписать коэффициенты зацепления +1 и -1 соответственно, имея в виду их продолжения до зацеплений $S^{2} \sqcup S^{2} \hookrightarrow S^{5}$ с помощью очевидных включений восьми дисков $\overline{S^{2} \backslash P} \sqcup \overline{S^{2} \backslash P}=\left(\bigsqcup_{j} D_{j}^{2}\right) \sqcup\left(\bigsqcup_{j} D_{j}^{2}\right)$ в четыре шара $\overline{S^{5} \backslash Q}=\bigsqcup_{j} B_{j}^{5}$. Зафиксируем какую-нибудь гомотопию между вложениями $g_{0} \sqcup g_{1}$ и $u \circ\left(g_{0} \sqcup g_{1}\right)$, для простоты, с единственной двойной точкой (конечно, трансверсальная гомотопия имела бы как минимум пару двойных точек). Можно считать, что образ носителя этой гомотопии лежит в малой окрестности некоторого отрезка, соединяющего две точки в $g_{0}(P)$ и $g_{1}(P)$ и лежашего вблизи $\partial B_{0}^{5}$, поэтому несложно добиться того, чтобы вся гомотопия происходила вне носителя трицикла $T$. С помощью гомеоморфизмов $\varphi_{*}$ и $\psi_{*}$ распространим ее до гомотопии $h_{t}:\left(D^{2} \sqcup D^{2}, \partial\right) \rightarrow\left(B^{5}, \partial\right)$ между отображениями $\bar{g}$ и $u \circ \bar{g}$; и далее, подкручивая гомеоморфизмами $\left.H\right|_{B^{5}}$ и $\left.h\right|_{D^{2}}$ (с которыми она перестановочна ввиду последнего замечания), на полнотория $\left(D^{2} \sqcup D^{2}\right) \times S^{1}$. Таким образом, последняя гомотопия соединяет отображения $G$ и $U \circ G$, где $U$ - обращающий ориентацию автогомеоморфизм $B^{5} \times S^{1}$, послойный по отношению к проекции на $S^{1}$, который получен из некоторого автогомеоморфизма $B^{5}$ закруткой со сдвигом на $\left.H\right|_{B^{5}}$.

Аналогичные построения возможны и при любом $n-1 \geqslant 3$. Представим полученную гомотопию как $j_{n}:\left(D^{n-2} \sqcup D^{n-2}\right) \times S^{1} \times I \rightarrow B^{2 n-3} \times S^{1} \times I$ и склеим нижнее основание с верхним по инволюциям $\mathfrak{s}_{n}$ и $\mathfrak{t}_{n} \circ U$ (если $n$ четно, $\mathfrak{t}_{n}$ обращает ориентацию, компенсируя тем самым гомеоморфизм $U$ ). Полученное отображение $J_{n}:\left(D^{n-2} \times S^{1} \times S^{1}, \partial\right) \rightarrow\left(B^{2 n-3} \times S^{1} \times S^{1}, \partial\right)$ имеет в качестве сингулярного множества, во-первых, произведение 3 -адического соленоида на окружность и, во-вторых, счетное число окружностей $\mathscr{O}$, накапливаюшихся к произведению соленоида на некоторую фиксированную точку $p t \in S^{1} .{ }^{4}$ Как и в примере 1 , отобра-

\footnotetext{
${ }^{3}$ Для установления полной аналогии с "перестановкой листов" отметим, что гомеоморфизм $\hat{\mathfrak{s}} n$ порядка 4 , меняющий местами два экземпляра $D^{n-2} \times S^{1}$ и затем осуществляющий центральную симметрию в каждом слое $D^{n-2} \times p t$ одного из них (тем самьм $\hat{\mathfrak{s}}_{n}^{2}$ сохраняет ориентацию, если и только если $n$ нечетно), реализуется посредством $G_{n}$ и некоторого гомеоморфизма $B^{2 n-3} \times S^{1}$ порядка 4 , сохраняющего ориентацию (проверка предоставляется читателю).

${ }^{4}$ В наблюдении 4.4 мы выясним, что появление этих окружностей алгебраически неслучайно, в некотором смысле без них нельзя обойтись в любом таком примере.
} 
жение $J_{n}$ легко продолжить до $\bar{J}_{n}: S^{n} \rightarrow \mathbb{R}^{2 n-1}$, которое в композиции со включением $\mathbb{R}^{2 n-1} \hookrightarrow \mathbb{R}^{n}$ будет реализуемо дискретно (в самом деле, на дополнении к добавочным окружностям $\mathscr{O}$ оно по построению дискретно реализуемо уже в гиперплоскости, а при обходе по каждой из этих окружностей листы не переставляются, так что в объемлющем пространстве их возможно распроектировать), но не изотопически (что проверяется непосредственно; см. замечание ниже). Ясно также, что $\bar{J}_{n}$ не утрачивает по сравнению с $\bar{G}_{n}$ свойства быть локально плоским топологическим погружением.

Пример $2^{\prime}$. Вернемся теперь к случаю нечетного $n$, в котором мы с первой попытки потерпели неудачу. Рассмотрим гладкое погружение бутылки Клейна $K \uparrow \mathbb{R}^{3} \subset \mathbb{R}^{2 n-1}$. Тотальное пространство его нормального расслоения допускает погружение $\rho: \bar{K} \uparrow \mathbb{R}^{2 n-1}$ с коразмерностью нуль.

Заметим теперь, что, если $n \geqslant 5$, любой гомеоморфизм $S^{n-2}$, неподвижный на дисках $D_{j}^{n-2}$ (определенных вьше в случае $n=4$ ), изотопен тождеству связанно на дисках, поскольку $\pi_{1}\left(S^{n-2}, \bigcup_{j=0}^{3} D_{j}^{n-2}\right)=1$. Следовательно, любой гомеоморфизм $S^{2 n-3}=S_{0}^{n-2} * S_{1}^{n-2}$, неподвижный на шарах $B_{j}^{2 n-3}$ и согласованный со структурой джойна, изотопен тождеству связанно на шарах и согласованно со структурой джойна. В частности, гомеоморфизмы $T^{-1}$ и $H_{12}^{-1} T H_{12}$, совпадающие на шарах в силу $(123)^{(12)}=(321)$, изотопны связанно на шарах и согласованно со структурой джойна. ${ }^{5}$ Стало быть, $T$ сопряжен $T^{-1}$ сохраняющим ориентацию гомеоморфизмом, переводящим каждое $g_{i}$ в себя и шары в шары согласно подстановке (12), с точностью до изотопии, связанной на шарах и переводящей каждое $g_{i}$ в себя. Это означает, что имеется послойный автогомеоморфизм тора гомеоморфизма $\left.T\right|_{Q^{2 n-3}}$ (напомним, $Q^{2 n-3}$ определялся выше в случае $n=4$ как $\left.\overline{S^{2 n-3} \backslash \bigcup_{j=0}^{3} B_{j}^{2 n-3}}\right)$, сохраняюший ориентацию слоя $Q^{2 n-3}$, обращающий ориентацию $S^{1}$ и переводящий в себя $\partial B_{0}^{2 n-3} \times S^{1}$, а также тор ограничения $T$ на образ каждого вложения $g_{i}$. Отсюда следует, что имеется послойный автогомеоморфизм $\omega$ тора гомеоморфизма $\left.H\right|_{B^{2 n-3}}\left(\right.$ где $\left.B^{2 n-3}=\overline{S^{2 n-3} \backslash B_{0}^{2 n-3}}\right)$, сохраняюший ориентацию слоя, на 3 -адическом соленоиде $\mathscr{D}^{\prime}$ совпадающий со стандартным автогомеоморфизмом соленоида, обращающим ориентацию $S^{1}$, и переводящий отображение $G_{n}$ в себя.

Рассмотрим теперь тор гомеоморфизма $\omega$. Поскольку тор гомеоморфизма $\left.H\right|_{B^{2 n-3}}$ гомеоморфен $B^{2 n-3} \times S^{1}$ и $\omega$ обращает ориентацию $S^{1}$, сохраняя ориентацию слоя, тор $\omega$ гомеоморфен $K \times B^{2 n-3}$. Следовательно, тор гомеоморфизма $\mathfrak{t}_{n} \circ U \circ \omega$ гомеоморфен $\bar{K}$ (поскольку $\mathfrak{t}_{n}$ сохраняет ориентацию при нечетном $n$, a $U$ обрашает ориентацию слоя), и гомотопия $j_{n}$ доставляет послойное отображение в $\bar{K}$ тора инволюции $\mathfrak{s}_{n}$, скомпонированной со сменой ориентации $S^{1}$, гомеоморфного $\left(D^{n-2} \sqcup D^{n-2}\right) \times S^{1} \times S^{1}$. Композиция последнего с погружением $\rho$ и есть, очевидно, искомое отображение.

ЗАМЕЧАНИЕ. В отношении проверки изотопической нереализуемости в приме$\operatorname{pax} 1,2$ и 2' заметим, что обратные последовательности из эквивариантных когомологий в этих примерах (вторая строчка в диаграмме) и из неэквивариантных ко-

\footnotetext{
${ }^{5} \mathrm{~B}$ случае $n=4$ последнее неверно; достаточно рассмотреть ограничения этих гомеоморфизмов на $S_{0}^{n-2}$.
} 
гомологий в примере 2 (первая строчка; ср. обсуждение последовательностей Смита в $\S 4)$, если пренебречь дополнительньми образующими, отвечающими окружностям $\mathscr{O}$ (они все равно не оказывают влияния на производный предел), выглядят каK

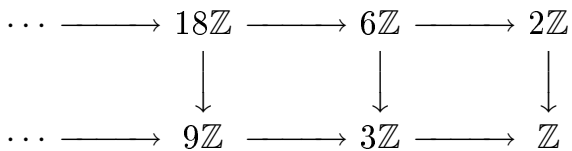

Неэквивариантная система для примера 2 имеет вид $x_{i}-3 x_{i+1}=2$ и разрешима (достаточно взять все $x_{i}$ равными -1 ), а эквивариантная, совпадая с системой $x_{i}-3 x_{i+1}=1$ для примера 1 , неразрешима (что непосредственно очевидно, см. [4] или [7]). См. также последнюю строчку в [5; приложение].

\section{§3. Немного вычислений}

ТЕОРЕма 3.1. Пусть $\gamma(n)$ обозначает 2-примарную часть того из чисел $n, n+1$, которое четно, и $N^{n}, M^{2 n-k}$ - стабильно параллелизуемые многообразия, причем $n \neq 2$ и $N$ компактно. Любое отобрахсение $\bar{f}: N \stackrel{f}{\longrightarrow} M \subset$ $M \times \mathbb{R}^{k}$ изотопически реализуемо при любом из следующих двух условий:

(a) $k \geqslant \gamma(n+1)$;

(б) $k \geqslant \gamma(n)+1$.

Приведем план доказательства. Наблюдения 3.2 и 3.4 доставляют две независимые редукции (приводящие в итоге к двум разным ответам в пп. (а) и (б)) задачи оценки первого препятствия к изотопической реализуемости к задаче оценки первого препятствия к аппроксимируемости вложением с заданной точностью. Последняя решается аналогично доказательству теоремы $1,(\mathrm{a})$, состоящему из комбинации [10] и когомологической версии $[11 ; \S 3]$. В первой работе дано геометрическое доказательство легкой части [17] теоремы Адамса о тривиальности инварианта Хопфа $h^{n}: \Pi_{n} \rightarrow \mathbb{Z} / 2$ (по поводу сложной части см. [18]), а во второй - гомологическое доказательство теоремы Ахметьева [6] о дискретной реализации в $\mathbb{R}^{2 n}$ отображения между параллелизуемыми $n$-многообразиями при условии $n>2$ и $h^{n}=0$ (идея этого доказательства близка оригинальным построениям [6], в которых, однако, опущены некоторые неочевидные рассуждения, в частности доказательство [11; лемма 9], а другие рассуждения можно упростить).

НАБЛЮДЕНИЕ 3.2. Пусть $f: X^{n} \rightarrow Q^{m}$ - отображсение компактного полиэдра в ориентируемое кусочно линейное многообразие $u \varepsilon: X \times[0,1) \rightarrow(0,1]$ - собственная функиия. Тогда $\vartheta(f)$ переходит в первое препятствие $\vartheta_{\varepsilon}(F)$ $\kappa$-аппроксимируемости вложсением отображения $F: X \times[0,1) \rightarrow Q \times[0,1)$, $(x, t) \mapsto(f(x), t)$, при некотором изоморфизме межсду группами, в которых они принимают значения.

Пусть $f: X^{n} \rightarrow Q^{m}$ - собственное отображение полиэдра (не обязательно компактного) в кусочно линейное многообразие и $\varepsilon: X \rightarrow(0,1]$ - собственная функция. Препятствие $\vartheta_{\varepsilon}(f)$, определяемое аналогично $\vartheta_{i}$ и $\vartheta$, принимает значение в группе $H_{\mathrm{skew}}^{m}\left(\widetilde{X}, U_{\varepsilon}\right)$, где $U_{\varepsilon}$ - замыкание дополнения до полиэдральной $\mathfrak{t}$-инвариантной окрестности $N_{\varepsilon} \cup \Delta_{X}$ множества $\Sigma_{F} \cup \Delta_{X}$ в $X \times X$ такой, что $\Sigma_{F ; 2 \varepsilon} \supset N_{\varepsilon} \supset \Sigma_{F ; \varepsilon}:=\{(x, y) \mid x \neq y, \operatorname{dist}(f(x), f(y))<\min (\varepsilon(x), \varepsilon(y))\}$. 
ЗАмечАнИЕ. Согласно [4; теорема 1.12] при $m-n \geqslant 3$ изотопическая реализуемость $f$ равносильна $\varepsilon$-аппроксимируемости $F$ вложением, однако это нам не потребуется.

ДокАЗАТЕЛЬСтво. Заметим, что $N=N_{\varepsilon} \cap \widetilde{X} \times \Delta_{[0,1)}$ отождествляется с дополнением в $\widetilde{X} \times[0,1)$ до телескопа $U$ из определения $\vartheta(f)$. Искомый гомоморфизм есть композиция изоморфизма Тома

$$
\left.H_{\text {skew }}^{m}\left(\tilde{X} \times \Delta_{[0,1)}, \tilde{X} \times \Delta_{[0,1)} \backslash N\right) \cong H_{\text {skew }}^{m+1}(\widetilde{X \times[0,1}), X \widetilde{\times[0,1)} \backslash N \times \mathbb{R}\right)
$$

и гомоморфизма расширения носителя

$$
\left.\left.\left.\left.H_{\text {skew }}^{m+1}(X \widetilde{\times[0,1}), X \widetilde{\times[0,1}\right) \backslash N \times \mathbb{R}\right) \rightarrow H_{\text {skew }}^{m+1}(X \widetilde{\times[0,1}), \widetilde{\times[0,1}\right) \backslash N_{\varepsilon}\right),
$$

где $N \times \mathbb{R}$ вложено в $N_{\varepsilon}$ эквивариантно относительно диагонального произведения инволюции на $N$ и инволюции $x \mapsto-x$ на $\mathbb{R}$. Он является изоморфизмом, поскольку дополнениек $N \times \mathbb{R}$ допускает деформационную ретракцию на дополнение к $N_{\varepsilon}$.

ЛЕмма 3.3. Рассмотрим композицию $\bar{f}: X^{n} \stackrel{f}{\longrightarrow} Q^{m} \subset Q \times \mathbb{R}^{k}$, әде $f$ отображает компактный полиәдр в ориентируемое кусочно линейное многообразие.

(а) (ср. [11; лемма 7]) Справедливо следующее: $\vartheta_{i}(\bar{f})=\vartheta_{i}(f) \smile e^{k}$, әде $е \in$ $H_{\text {skew }}^{1}(\widetilde{X}) \simeq H^{1}\left(\widetilde{X} / \mathfrak{t} ; \mathscr{O}_{\mathbb{Z}_{\mathrm{T}}}\right)$ - класс Эйлера двулистного накрытия $\widetilde{X} \rightarrow \widetilde{X} / \mathfrak{t}$. Более того, $\vartheta(\bar{f})=\vartheta(f) \smile e^{k}$.

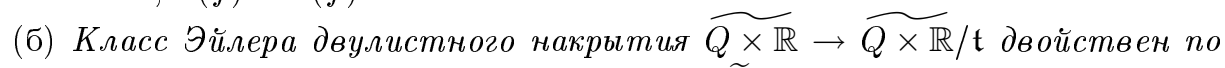
Пуанкаре локально конечной гомологии $\left[\Delta_{\mathbb{R}} \times \widetilde{Q}\right]$.

(в) (ср. [11; лемма 6]) Пусть $g: X \hookrightarrow Q \times \mathbb{R}^{k}$ - вложение, $2^{-i-1}$-близкое к $\bar{f}$, и класс $\xi_{\text {skew }}^{m} \in H_{\text {skew }}^{m}\left(\widetilde{Q \times \mathbb{R}^{k}}, \widetilde{Q}\right)$ двойствен по Пуанкаре локально конечной гомологии $\left[\Delta_{Q} \times \mathbb{R}^{k}\right]$. Тогда обратный образ $\left(g^{2}\right)_{\text {skew }}^{*}\left(\xi_{\text {skew }}^{m}\right) \in H_{\text {skew }}^{m}\left(\widetilde{X}, U_{i}\right)$ совпадает с $\vartheta_{i}(f)$.

ЗАмечАниЕ. Для $Q=\mathbb{R}^{m}, k=1$ утверждение п. (в) состоит в том, что $\vartheta_{i}(f)$ совпадает с обратным образом образуюшей $H_{\text {skew }}^{m}\left(S^{m}, S^{m-1}\right) \cong \mathbb{Z}$ при отображении $\widetilde{g}$.

ЗАмЕчАниЕ. Легко видеть, что при $\smile$-произведении набор символов $\{$ eq, skew $\}$ ведет себя как группа $\mathbb{Z} / 2$ (с образующей еq), т.е. произведение двух классов, один из которых лежит в еq-когомологиях, а другой в skew-когомологиях, попадает в eq-когомологии, а произведение двух однотипных классов - в skew-когомологии.

ЗАмЕчАниЕ. Поскольку класс Эйлера $е$ имеет порядок 2, второе утверждение леммы $3.3,(\mathrm{a})$ доставляет альтернативное доказательство предложения 2.2 .

ДокАЗАТЕльство. (в) По определению препятствия $\vartheta_{i}(f)$ оно совпадает с образом $\vartheta_{i+1}(p g)$ при гомоморфизме расширения носителя, где $p: Q \times \mathbb{R}^{k} \rightarrow Q$-проекция. Поэтому достаточно рассмотреть случай $f=p g$, причем $f$ можно считать отображением общего положения. Пусть $T$ - соответствуюшая триангуляпия $X$ (см. определение в $\S 1$ ). Тогда коцикл, по определению представляющий $\vartheta_{i}(f)$, принимает ненулевое значение на клетке $\sigma \times \tau$, где $\sigma^{s}, \tau^{t} \in T, s+t=m$, тогда и 
только тогда, когда найдутся точки $x \in g(\sigma)$ и $y \in g(\tau)$ такие, что $p(x)=p(y)$; эквивалентно, $(x, y) \in \Delta_{Q} \times \widetilde{\mathbb{R}^{k}}$. Таким образом, в рассматриваемом случае утверждаемое совпадение вьполнено уже на уровне коцепей.

(б) Поскольку указанный класс Эйлера есть обратный образ класса Эйлера

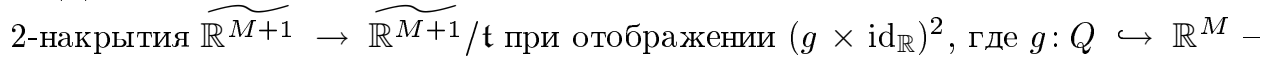
какое-нибудь вложение, достаточно рассмотреть случай $Q=\mathbb{R}^{m}$. Эквивариантная гомотопическая эквивалентность $\widetilde{\mathrm{id}}: \widehat{\mathbb{R}^{m+1}} \rightarrow S^{m}$ сводит задачу к нахождению класса Эйлера универсального $\mathbb{Z} / 2$-расслоения $p: S^{m} \rightarrow \mathbb{R} P^{m}$, которьй, как известно, двойствен по Пуанкаре гомологии $\left[\mathbb{R} P^{m-1}\right]$. В самом деле, ассоциированное с $p$ универсальное линейное расслоение $\gamma: E \rightarrow \mathbb{R} P^{m}$ допускает сечение, обращающееся в нуль на $\mathbb{R} P^{m-1}$, которое может быть получено совмещением антиподальных точек края северного полушария $S^{m} \subset E$.

(a) Достаточно рассмотреть случай $k=1$; положим $l=2 n-m+1$. По обшему положению найдется вложение $g: X \hookrightarrow Q \times \mathbb{R}^{l}$, достаточно близкое к композиции $f$ со включением $Q \subset Q \times \mathbb{R}^{l}$. Согласно п. (в) класс $\vartheta_{i}(f)$ является обратным образом $\xi_{\text {skew }}^{m} \in H_{\text {skew }}^{m}\left(\widetilde{Q \times \mathbb{R}^{l}}, \widetilde{Q}\right)$ при отображении $g^{2}$. Аналогично, класс $\vartheta_{i}(\bar{f})$ является обратным образом $\xi_{\mathrm{skew}}^{m+1} \in H_{\mathrm{skew}}^{m+1}\left(\widetilde{Q \times \mathbb{R}^{l}}, \widetilde{Q \times \mathbb{R}}\right)$ при отображении $g^{2}$. Поскольку образ $\bar{f}$ содержится в $Q$, класс $\vartheta_{i}(\bar{f})$ можно также индуцировать из $\Xi_{\text {skew }}^{m+1} \in H_{\text {skew }}^{m+1}\left(\widetilde{Q \times \mathbb{R}^{l}}, \widetilde{Q}\right)$, являюшегося образом $\xi^{m+1}$ при гомоморфизме расширения носителя. Но $\left[\Delta_{Q \times \mathbb{R}} \times \widetilde{\mathbb{R}^{l-1}}\right]=\left[\Delta_{Q} \times \widetilde{\mathbb{R}^{l}}\right] \frown e_{0}$, где $e_{0} \in H_{\text {skew }}^{1}\left(\widetilde{Q \times \mathbb{R}^{l}}\right)$

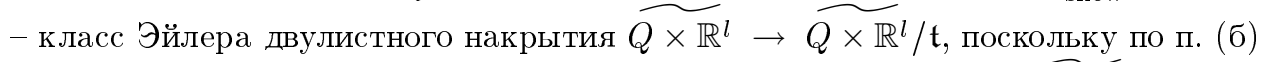
$e_{0}$ двойствен по Пуанкаре локально конечной гомологии $\left[\Delta_{\mathbb{R}} \times Q \widehat{\times \mathbb{R}^{l-1}}\right.$. Значит, $\Xi_{\text {skew }}^{m+1}=\xi_{\text {skew }}^{m} \smile e_{0}$, что доказывает первое утверждение п. (a), так как $e=\left(g^{2}\right)_{\text {skew }}^{*}\left(e_{0}\right)$ ввиду эквивариантности $g^{2}$.

Второе утверждение п. (а) является следствием наблюдения 3.2 и собственной (некомпактной) версии первого.

В случае $m=2 n-1$ следующее утверждение является усилением аналогичного случая теоремы 2.1 (по модулю теоремы 1.2), причем ни одно из двух доказательств этого случая теоремы 2.1 (из [7] и из $§ 2$ ) не удается модифицировать для получения доказательства усиления.

НАБЛЮДЕНИЕ 3.4. Рассмотрим композищию $\bar{f}: X^{n} \stackrel{f}{\rightarrow} Q^{m} \subset Q \times \mathbb{R}$, әде $f$ - отображение компактного полиэдра в ориентируемое кусочно линейное многообразие. Если $\check{\vartheta}(f)=0$, то $\vartheta(\bar{f})=0$.

Дальнейшее уточнение этого результата, выражаюшее $\vartheta(\bar{f})$ как функцию от $\check{\vartheta}(f)$, будет дано в $\S 4$.

ДокАЗАТЕЛьство. Пусть $N=\left\{1-1 / 2^{n} \mid n \in \mathbb{N}\right\}$ и $U$ - телескоп из определения препятствия $\vartheta(f)$ в $\S 1$ (см. также доказательство наблюдения 3.2 ). Точная последовательность тройки вместе с изоморфизмом

$$
H_{\text {skew }}^{i}(\widetilde{X} \times[0,1), \widetilde{X} \times N \cup U) \cong H_{\text {skew }}^{i-1}\left(\widetilde{X} \times N, \bigsqcup U_{i}\right)
$$


доставляют коммутативную диаграмму

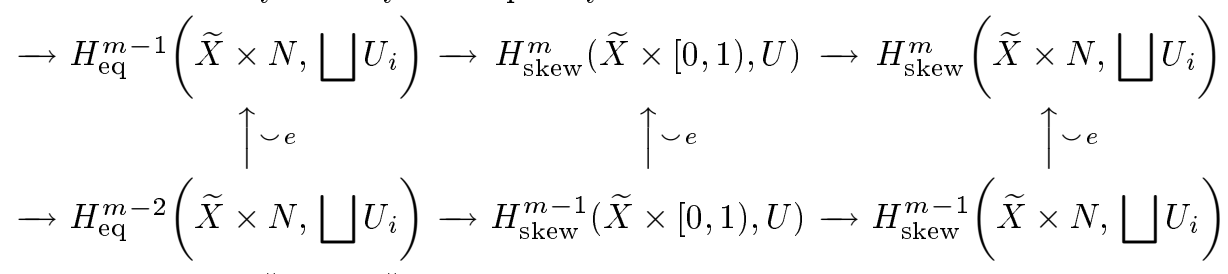

По лемме $3.3,($ a) $\breve{\vartheta}(\bar{f})=\breve{\vartheta}(f) \smile e=0$ в образе правого верхнего гомоморфизма, следовательно, как показано при доказательстве критерия $1.3, \vartheta(\bar{f})$ отождествляется с элементом из коядра левой верхней стрелки, представленным в левой верхней группе набором коэффициентов $\left\{d_{\text {eq }}\left(g_{i}, g_{i+1}\right)\right\}$ системы $(1)$, причем элементы образа левой верхней стрелки суть наборы коэффициентов разрешимых систем. По лемме $3.3,($ a) имеем также $\vartheta(\bar{f})=\vartheta(f) \smile e$. Поскольку $\breve{\vartheta}(f)=0$, препятствие $\vartheta(f)$ приходит из некоторого элемента $c$ левой нижней группы. Следовательно, элемент $c \smile e=\left\{d_{i}\right\}$ отличается от $\left\{d_{\mathrm{eq}}\left(g_{i}, g_{i+1}\right)\right\}$ на элемент из образа левой верхней стрелки, т.е. система $x_{i}-\pi_{i} x_{i+1}=d_{\mathrm{eq}}\left(g_{i}, g_{i+1}\right)-d_{i}$ разрешима. Но каждьй класс $d_{i}$ имеет порядок 2 ввиду $2 e=0$, поэтому система $x_{i}-\pi_{i} x_{i+1}=d_{i}$ также разрешима. Сумма решений этих двух систем есть решение системы (1).

ПреДЛОЖЕНИЕ 3.5 (ср. [3; теорема 1], [11; §3]). Рассмотрим композицию $\bar{\varphi}: N^{n} \stackrel{\varphi}{\longrightarrow} M^{2 n-\gamma(n)-1} \subset M \times \mathbb{R}^{\gamma(n)}$, где $\varphi-$ собственное гладкое погружение межсду стабильно параллелизуемыми многообразиями (функиия ү определена в теореме 3.1$)$, причем либо $\gamma(n)+1<n / 2$, либо нормальное расслоение $\nu(\varphi)$ тривиально. Тогда $\vartheta_{\varepsilon}(\bar{\varphi})=0$ для любой собственной функиии $\varepsilon: N \rightarrow(0,1]$.

ДоКАЗАТЕЛЬСТво. Положим $k=\gamma(n)+1$; поскольку в случае, если $n$ или $n+1$ является степенью двойки, утверждение выполнено по размерностным соображениям (см. введение), мы можем исключить этот случай из рассмотрения, так что $k<n$. По лемме $3.3,($ а $)$ класс $\vartheta_{\varepsilon}(\bar{\varphi})$ является произведением $\vartheta_{\varepsilon}(\varphi)$ на $(k-1)$-ю степень $e^{k-1} \in H_{\text {skew }}^{k}(\widetilde{N})$ класса Эйлера двулистного накрытия $p: \widetilde{N} \rightarrow \widetilde{N} / \mathfrak{t}$. В частности, $\vartheta_{\varepsilon}(\bar{\varphi})$ является тогда элементом порядка 2 , поэтому можно перейти к коэффициентам, приведенным по модулю 2. Поскольку 乙-умножение коммутативно $\bmod 2$, изоморфизм Тома

$H^{i}\left(\Sigma_{\varphi} / \mathfrak{t} ; \mathbb{Z} / 2\right) \simeq H^{i}(E ; \mathbb{Z} / 2) \stackrel{\smile u}{\longrightarrow} H^{i+2 n-k}(E, \partial E ; \mathbb{Z} / 2) \simeq H^{i+2 n-k}(\tilde{N} / \mathfrak{t}, V ; \mathbb{Z} / 2)$, где $V$ - замыкание дополнения к трубчатой окрестности $E$ гладкого подмногообразия $\Sigma_{\varphi} / \mathfrak{t} \subset \widetilde{N} / \mathfrak{t}$, перестановочен с умножением на $(k-1)$-ю степень первого класса Штифеля-У итни $w_{1} \in H^{1}(E ; \mathbb{Z} / 2)$ двулистного накрытия $p^{-1}(E) \rightarrow E$. Таким образом, ввиду $w_{1}=\left.e\right|_{E}(\bmod 2)$ остается проверить, что $w_{1}$ или, эквивалентно, первый класс Штифеля-Уитни двулистного накрытия $\Sigma_{\varphi} \rightarrow \Sigma_{\varphi} / \mathfrak{t}$ не выживает при возведении в $(k-1)$-ю степень. Но это вытекает из случая $k=2^{l}+1 \geqslant 3$ следующей леммы, поскольку $n \equiv \gamma(n)$ или $\gamma(n)-1(\bmod 2 \gamma(n))$.

Лемма 3.6 (ср. [35], [10], [36]). Пусть $\varphi: N^{n} \rightarrow M^{2 n-k}, n>k,-c o б c m-$ венное гладкое погружение общего положения между стабильно параллелизуемыми многообразиями, $k=2^{l}$ или $k=2^{l}+1 \geqslant 3$, и либо $n>2 k$, либо $\nu(\varphi)$ тривиально, причем остаток при делении $n-k$ на $2^{l+1}$ есть одно из чисел $2^{l}, 2^{l}+1, \ldots, 2^{l+1}-1$. Тогда $w_{1}^{2^{l}}=0$, где $w_{1}=w_{1}(\eta)$ обозначает первий класс Штифеля-Уитни одномерного линейное расслоения $\eta$, ассочичрованного с двулистным накрытием $\Sigma_{\varphi} \rightarrow \Sigma_{\varphi} / \mathfrak{t}$. 
ДокАЗАТЕЛьство. Согласно [35; лемма 2] в случае $n>2 k$ и [15; с. 202] в случае $\nu(\varphi)=(n-k) \varepsilon$ нормальное расслоение $\nu\left(\varphi^{(2)}\right)$ к погружению двойных точек $\varphi^{(2)}: \Sigma_{\varphi} / \mathfrak{t} \leftrightarrow M$, определяемому по формуле $\{x, y\} \mapsto \varphi(x)=\varphi(y)$, представляется в виде суммы Уитни $\nu=(n-k)(\varepsilon \oplus \eta)$, где $\varepsilon$ - одномерное тривиальное расслоение. По формуле Уитни $w_{2^{l}}(\nu)=C_{n-k}^{2^{l}} w_{1}^{2^{l}}(\eta)$, где биномиальный коэффициент

$$
C_{n-k}^{2^{l}}=\frac{(n-k) \cdots\left(n-k-2^{l}+1\right)}{2^{l} !} \equiv \frac{\cdots\left(2^{l}-1\right) \cdot 2^{l} \cdot 1 \cdot 2 \cdot 3 \cdots}{1 \cdot 2 \cdot 3 \cdots\left(2^{l}-1\right) \cdot 2^{l}} \quad\left(\bmod 2^{l}\right),
$$

поскольку, принимая во внимание условие, ни одно из $2^{l}$ чисел $n-k, \ldots, n-k-$ $2^{l}+1$ в числителе не делится на $2^{l+1}$. В частности, $C_{n-k}^{2^{l}} \equiv 1(\bmod 2)$ и, значит, $w_{1}^{2^{l}}=w_{2^{l}}(\nu)=0$, так как по теореме Масси [37] для нормального расслоения $\nu$ произвольного $k$-мерного многообразия $w_{k}(\nu)=0, \ldots, w_{k-\alpha(k)+1}(\nu)=0$, где $\alpha(k)$ - количество единиц в двоичной записи числа $k$ (в случае $k=2^{l}+1$ здесь используется равенство $\alpha(k)=2$, что объясняет ограничение $k \geqslant 3$ ).

ЗАМЕЧАНИЕ. По сути мы доказывали тривиальность композиции

$\check{\Omega}_{k}^{\mathrm{fr} ;(\mathbb{Z} / 2) \times B_{\infty} ; \text { lf }}\left(\Sigma_{f} ; F_{n-k}\right) \rightarrow \check{\Omega}_{1}^{\mathrm{fr} ;(\mathbb{Z} / 2) \times B_{\infty} ; \text { lf }}\left(\Sigma_{f} ; F_{n-1}\right) \rightarrow \check{H}_{1}^{\mathbb{Z} / 2 ; \text { lf }}\left(\Sigma_{f} ; \mathbb{Z}_{\mathrm{T}}^{\otimes(n-1)}\right)$

гомоморфизмов, определенных в [16] (по поводу обозначений см. [5; приложение]).

ДоКАЗАТЕЛЬСТво тЕОремЫ 3.1. (б) Согласно $C^{0}$-плотному $h$-принципу Хирша (в смысле Громова [38; 1.2 .2 (А), примеры (2)], см. также [39; 4.4]) произвольное отображение $f: N \rightarrow M$ аппроксимируется сколь угодно близко гладким погружением $\varphi: N$ q $M$ общего положения с тривиальным нормальным расслоением. Предложение 3.5 устанавливает тривиальность $\vartheta_{i+1}(\bar{\varphi})$, где $\bar{\varphi}-$ композиция $\varphi$ и включения $M \subset M \times \mathbb{R}^{k-1}$ и предполагается, что $\varphi 2^{-i-1}$-близко к $f$. По определению препятствия ван Кампена-Скопенкова это означает, что и $\vartheta_{i}(\bar{f})=0$, где $\bar{f}-$ композиция $f$ и включения $M \subset M \times \mathbb{R}^{k-1}$. Следовательно, ввиду наблюдения 3.4 и теоремы $1.2,(б) \bar{f}$ изотопически реализуемо.

(a) Согласно [3; лемма 2] существует гладкая локальная изотопическая реализация отображения $f$, т.е. гладкая регулярная гомотопия $f_{t}: N \leftrightarrow M, t \in[0,1)$, такая, что $f_{t} \rightarrow f$ равномерно при $t \rightarrow 1$. Рассматривая ее как собственное гладкое погружение $F: N \times[0,1) \rightarrow M \times[0,1)$, которое, не ограничивая общности, можно взять в общем положении и с тривиальным нормальным расслоением, применим предложение 3.5 , заключив, что $\vartheta_{\varepsilon}(\bar{F})=0$ для композиции $\bar{F}$ погружения $F$ со включением $M \times[0,1) \subset M \times \mathbb{R} \times[0,1)$ и собственной функции $\varepsilon: N \times[0,1) \rightarrow(0,1]$, определяемой по формуле $(x, t) \mapsto d(f(x), F(x, t))$. По определению препятствия ван Кампена-Скопенкова и $\vartheta_{2 \varepsilon}\left(\bar{f} \times \mathrm{id}_{[0,1)}\right)=0$. Как показано в наблюдении 3.2 , $\vartheta_{2 \varepsilon}\left(\bar{f} \times \operatorname{id}_{[0,1)}\right)$ можно отождествить с $\vartheta(\bar{f})$, значит, $\bar{f}$ изотопически реализуемо по теореме $1.2,(б)$.

\section{§4. Отображения $S^{n} \rightarrow S^{n} \subset \mathbb{R}^{2 n}$}

Основной целью параграффа является доказательство теоремы 2,(а) (в когомологической версии) при одном дополнительном предположении, устранению которого посвящены следующие два параграфа. Следующие две леммы, которые можно рассматривать как дополняющие пп. (б) и (а) леммы 3.3, вместе с наблюдением 2.3 доказывают когомологическую версию теоремы $2,($ б). 
Лемма 4.1 [11; лемма 8]. Существует әквивариантная гомотопическая әквивалентность $\varphi: \widetilde{S^{n}} \rightarrow S^{n}$, трансверсальная $к S^{0}$ и такая, что $\varphi^{-1}\left(S^{0}\right)=$ $\left(\{q\} \times S^{n} \cup S^{n} \times\{q\}\right) \backslash\{(q, q)\}$.

Лемма 4.2. Рассмотрим композицию $\bar{f}: X^{n} \stackrel{f}{\longrightarrow} Q^{m} \subset Q \times \mathbb{R}^{k}$, где $f$ отображает полиэдр в ориентируемое кусочно линейное многообразие, $и$ әквивариантное отображение $\varphi: N_{\varepsilon} \rightarrow S^{N}$, трансверсальное $\kappa S^{N-k}$, для некоторой собственной функции $\varepsilon: X \rightarrow(0,1]$ (окрестность $N_{\varepsilon}$ определена $\boldsymbol{\theta} \S 3)$.

(a) Eсли $m>\frac{3}{2} n$, найдется $\varepsilon$-аппроксимация $g$ общего положения отображения $\bar{f}$ такая, ито $\Sigma_{g} \subset \varphi^{-1}\left(S^{N-k}\right)$.

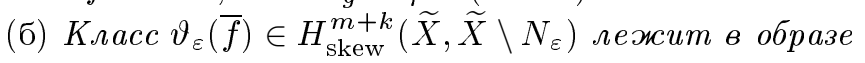

$$
H_{\text {skew }}^{m+k}\left(\widetilde{X}, \widetilde{X} \backslash \varphi^{-1}\left(S^{N-k}\right)\right) \text {. }
$$

ЗАмечАниЕ. Если $X$ - PL-многообразие, отображение $\varphi$ можно восстановить (с точностью до малой гомотопии) по его ограничению на объединение $\Sigma_{f}$ со сферизацией касательного расслоения $X$, отождествленной с короной частичной (если $X$ некомпактно) компактификации $\widetilde{X}$. В самом деле, если $\varepsilon$ достаточно мала, по лемме Урысона композиция этого ограничения и накрытия $S^{N} \rightarrow \mathbb{R} P^{N}$ продолжается на $N_{\varepsilon}$, причем с помошью исходного ограничения это продолжение поднимается до эквивариантного отображения $N_{\varepsilon} \rightarrow S^{N}$.

ДоказАтельство. Пусть $p:\left(S^{N}, S^{N-k}\right) \subset\left(\mathbb{R}^{N+1}, \mathbb{R}^{N+1-k}\right) \rightarrow\left(\mathbb{R}^{k},\{0\}\right)$ - композиция стандартного включения и проекции, эквивариантная относительно центральных симметрий. Рассмотрим $\varepsilon$-аппроксимацию $F$ общего положения отображения $f$, а также отображение $\psi: \Sigma_{F} \rightarrow \mathbb{R}^{k}$, определенное по формуле $(x, y) \mapsto \operatorname{dist}(x, y) p \varphi(x, y)$, где предполагается, что метрика на $X$ (быть может, не полная) такова, что расстояние между любыми двумя точками не превосходит 1.

Если $m>\frac{3}{2} n$, отображение $F$ не имеет тройных точек (и тем более двойных точек на зонтиках), поэтому ограничение $h: \Sigma_{F} \rightarrow h\left(\Sigma_{F}\right)$ проекции $X \times X$ на первьй сомножитель является гомеоморфизмом. Положим $S_{F}=\overline{h\left(\Sigma_{F}\right)}$ и зададим непрерывное отображение $\chi: S_{F} \rightarrow \mathbb{R}^{k}$ по формуле $x \mapsto \varepsilon(x) \psi h^{-1}(x)$, если $x \in$ $h\left(\Sigma_{F}\right)$, и $x \mapsto 0$ в противном случае. Тогда совместное отображение

$$
\left.F\right|_{S_{F}} \times \chi: S_{F} \rightarrow Q \times \mathbb{R}^{k}
$$

является вложением на дополнении к $\psi^{-1}(0)$, так как если две точки $S_{F}$ склеиваются отображением $F$, то они переходят в центрально симметричные при отображении $\psi h^{-1}$. Следовательно, продолжив $\chi$ произвольньм образом на $X$ с сохранением $\|\chi\| \leqslant \varepsilon$, мы получим, что $g:=F \times \chi$ является $\varepsilon$-близким к $\bar{f}$ и $\Sigma_{g}=\psi^{-1}(0)$.

Если $m \leqslant \frac{3}{2} n$, тройные точки нельзя исключить, поэтому требуется найти эквивариантную $\widehat{\varepsilon}$-аппроксимацию $G$ общего положения отображения $\bar{f}^{2}$, где $\widehat{\varepsilon}(x, y)=\min \{\varepsilon(x), \varepsilon(y)\}$, такую, что $G^{-1}\left(\Delta_{Q \times \mathbb{R}^{k}}\right) \subset \varphi^{-1}\left(S^{N-k}\right) \cup \Delta_{X}$. Для этого определим непрерывное отображение $\widehat{\chi}: \Sigma_{F} \cup \Delta_{X} \rightarrow \mathbb{R}^{k}$ по формуле $z \mapsto \widehat{\varepsilon}(z) \psi(z)$, если $z \in \Sigma_{F}$, и $z \mapsto 0$ в противном случае и продолжим его произвольным образом на $X \times X$ с сохранением $\|\chi\| \leqslant \widehat{\varepsilon}$. Совместное отображение $F^{2} \times \widehat{\chi}: X \times X \rightarrow Q \times$ $Q \times \nabla_{\mathbb{R}^{k}}$ является $\widehat{\varepsilon}$-близким к $\bar{f}^{2}$ и $\Sigma_{F^{2} \times \hat{\chi}}=\psi^{-1}(0)$.

Перейдем к доказательству п. (а) теоремы 2, которое будет продолжаться вплоть до конца §6. Нам понадобится несколько определений из теории Смита. 
Последовательности Смита (в случае $p=2, F=\varnothing$ ). Пусть $K$ - локально компактный полиэдр, на котором задано свободное кусочно линейное действие $\mathbb{Z} / 2$, и $L-$ его инвариантный подполиэдр (возможно пустой). Имеют место короткие точные последовательности $\mathbb{Z}[\mathbb{Z} / 2]$-модулей

$$
\begin{aligned}
& 0 \rightarrow \mathbb{Z}_{\mathrm{T}} \rightarrow \mathbb{Z}[\mathbb{Z} / 2] \rightarrow \mathbb{Z} \rightarrow 0, \\
& 0 \rightarrow \mathbb{Z} \rightarrow \mathbb{Z}[\mathbb{Z} / 2] \rightarrow \mathbb{Z}_{\mathrm{T}} \rightarrow 0 .
\end{aligned}
$$

Мономорфизм из первой (соответственно второй) последовательности переводит образуюшую в $1-\mathfrak{t}($ соответственно $1+\mathfrak{t})$. Эквивалентным образом можно рассматривать короткие точные последовательности локально постоянных пучков, ассоциированных с этими модулями. При любом подходе этим коэффициентным последовательностям отвечают две длинные точные последовательности когомологий, называемые последовательностями Смита [27; § 4], [22]:

$$
\begin{aligned}
\cdots \rightarrow H_{\mathrm{eq}}^{m-1}(K, L) \rightarrow H_{\mathrm{eq}}^{m}(K, L) & \rightarrow H^{m}(K, L) \\
& \rightarrow H_{\mathrm{skew}}^{m}(K, L) \rightarrow H_{\mathrm{skew}}^{m+1}(K, L) \rightarrow \cdots, \\
\cdots \rightarrow H_{\mathrm{skew}}^{m-1}(K, L) \rightarrow H_{\mathrm{skew}}^{m}(K, L) & \rightarrow H^{m}(K, L) \\
& \rightarrow H_{\mathrm{eq}}^{m}(K, L) \rightarrow H_{\mathrm{eq}}^{m+1}(K, L) \rightarrow \cdots
\end{aligned}
$$

Действительно, группа $H_{\mathbb{Z} / 2}^{m}(K, L ; \mathbb{Z}[\mathbb{Z} / 2])$ изоморфна $H^{m}(K, L ; \mathbb{Z})$ (мы опускаем целочисленные коэффициенты в неэквивариантных когомологиях), что с точки зрения модулей есть частньй случай изоморфизма

$$
H_{\pi}^{*}\left(K, L ; \mathbb{Z} \pi \otimes_{\mathbb{Z}} G\right) \simeq H^{*}(K, L ; G)
$$

(см. [5; приложение]), а с точки зрения пучков - частный случай теоремы Вьеториса-Бегля [22].

Таким образом, эти последовательности получены из коротких точных последовательностей коцепных комплексов

$$
\begin{aligned}
& 0 \longrightarrow C_{-}^{*} \stackrel{f_{-}}{\longrightarrow} C^{*} \stackrel{s_{+}}{\longrightarrow} C_{+}^{*} \longrightarrow 0, \\
& 0 \longrightarrow C_{+}^{*} \stackrel{f_{+}}{\longrightarrow} C^{*} \stackrel{s_{-}}{\longrightarrow} C_{-}^{*} \longrightarrow 0 .
\end{aligned}
$$

Здесь $C^{*}$ - обычный коцепной комплекс пары $(K, L)$, комплекс $C_{+}^{*}$ состоит из "симметричных" коцепей, т.е. инвариантных относительно действия инволюции $\mathfrak{t}$, a комплекс $C_{-}^{*}$ - из "кососимметричных", т.е. меняюших знак под действием $\mathfrak{t}$; гомоморфизмы коцепных комплексов $f_{-}$и $f_{+}$соответствуют "забыванию" (косо)симметричной структуры, $s_{+}$и $s_{-}$обозначают "симметризацию" $c \mapsto c+t c$ и "альтернацию" $c \mapsto c-t c$. Мы используем эти названия для обозначения гомоморфизмов в последовательностях Смита (аргумент $(K, L)$, общий для всех членов, опушен):

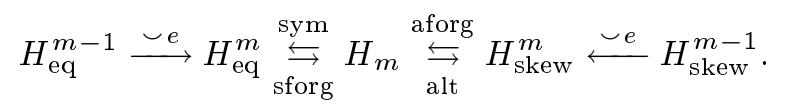

Легко видеть, что повышающие размерность гомоморфизмы суть умножения на класс Эйлера $e(\mathfrak{t}) \in H_{\text {skew }}^{1}(K) \simeq H^{1}\left(K / \mathfrak{t} ; \mathscr{O}_{\mathbb{Z}_{\mathrm{T}}}\right)$ инволюции $\mathfrak{t}$ (т.е. одномерного 
векторного расслоения над $K / \mathfrak{t}$, сферизация которого есть двулистное накрытие $K \rightarrow K / \mathfrak{t})$. Этот класс можно индуцировать из класса Эйлера

$$
e_{0} \in H^{1}\left(\mathbb{R} P^{\infty} ; \mathscr{O}_{\mathbb{Z}_{\mathrm{T}}}\right)=\mathbb{Z} / 2
$$

канонического линейного расслоения над $\mathbb{R} P^{\infty}=B(\mathbb{Z} / 2)$, сферизация которого есть двулистное накрытие $S^{\infty} \rightarrow \mathbb{R} P^{\infty}$, классифицируюшее расслоения со структурной группой $\mathbb{Z} / 2$. В частности, класс Эйлера $e(\mathfrak{t})$ есть элемент порядка 2 , однако поскольку он лежит в иелочисленной группе одномерных когомологий $K / \mathfrak{t}$ с локальными коэффициентами, мы будем отличать его от первого класса Штифеля-У итни $w_{1}(\mathfrak{t})$, принимаюшего значение в групте $H^{1}(K / \mathfrak{t} ; \mathbb{Z} / 2)$ и являюшегося образом $e(\mathfrak{t})$ при гомоморфизме приведения коэффициентов по модулю 2.

ЗАмЕчАниЕ. Из приведенного выше описания коцепных комлексов ясно, что:

(i) sym o sforg есть умножение на 2 в группе $H_{\mathrm{eq}}^{m}(K, L)$;

(ii) alt o aforg есть умножение на 2 в группе $H_{\text {skew }}^{m}(K, L)$;

(iii) sforg o sym + aforg ○ alt есть умножение на 2 в группе $H^{m}(K, L)$.

Последнее свойство следует из того, что инволюция $\mathfrak{t}$ индуцирует на группе $H^{m}(K, L)$ структуру $\mathbb{Z}[\mathbb{Z} / 2]$-модуля, причем $\operatorname{sforg} \circ \operatorname{sym}=\mathrm{id}+\mathfrak{t}^{*}$ и aforg $\circ$ alt $=\mathrm{id}-\mathfrak{t}^{*}$. Ввиду того что рассмотрения настоящего параграфа будут уточняться далее для случая отображения, пропущенного через подмногообразие коразмерности $k>1$, мы будем избегать чрезмерного использования перечисленных свойств.

ЛЕмма 4.3. Эпиморфизм обратных последовательностей $G_{i} \rightarrow G_{i} / \mathrm{ime}$, əде $G_{i}=H_{\mathrm{eq}}^{m}\left(\widetilde{X}, U_{i}\right)$, индуиирует изоморфизм $h: \lim ^{1} G_{i} \rightarrow \lim ^{1}\left(G_{i} / \operatorname{im} e\right)$.

ДоКАЗАТЕЛЬСтво. Поскольку класс Эйлера $e$ - элемент второго порядка (или в силу свойства (i) выше), im $e=\operatorname{ker}$ sforg лежит в 2-кручении $G_{i}$ для каждого $i$. Следовательно, $\lim ^{1}(\mathrm{im} e)=0$ (см. $\S 1$ ), так что утверждение следует из точности справа функтора производного предела (т.е. тривиальности $\lim ^{2}$ ).

НАБЛЮДЕНИЕ 4.4. Пусть $X^{n}-$ компактнъцй полиэдр, $Q^{m-1}-$ ориентируемое PL-многообразие и композиция $\bar{f}: X \stackrel{f}{\longrightarrow} Q \subset Q \times \mathbb{R}$ дискретно реализуема. Тогда $\vartheta(\bar{f})=h^{-1} \delta^{*}(\check{\vartheta}(f))$, где $h-$ изоморфизм из леммы 4.3, а $\delta^{*}-$ связывающий гомоморфизм из точной последовательности

$0 \longrightarrow \lim _{\longleftarrow} A_{i} \longrightarrow \lim _{\longleftarrow} B_{i} \longrightarrow \lim _{\longleftarrow} C_{i} \stackrel{\delta^{*}}{\lim ^{1}} A_{i} \longrightarrow \lim ^{1} B_{i} \longrightarrow \lim ^{1} C_{i} \longrightarrow 0$,

построенной по короткой точной последовательности обратных последовательностей

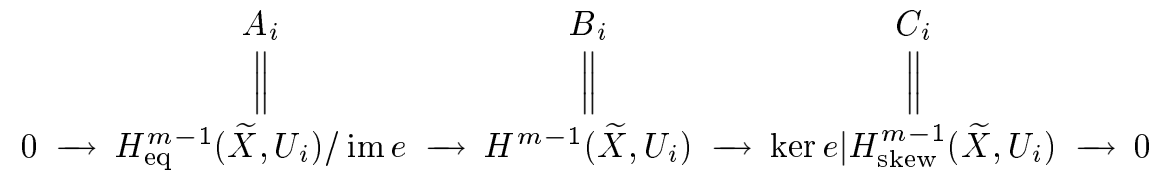


ДоказАТЕЛЬСтво. Прежде всего заметим, что $\operatorname{sforg} d_{\mathrm{eq}}\left(g_{i}, g_{i+1}\right)$ есть обычное неэквивариантное первое препятствие $d\left(g_{i}, g_{i+1}\right) \in H^{m-1}\left(\widetilde{X}, U_{i}\right)$ к гомотопии. По лемме 2.3 для него выполнено

$$
d\left(g_{i}, g_{i+1}\right)=\left(g_{i}^{2}\right)^{*}(\xi)-\left(g_{i+1}^{2}\right)^{*}(\xi)
$$

(где в случае $Q=\mathbb{R}^{m-1} \xi$ - одна из образующих $H^{m-1}\left(S^{m-1}, S^{m-2}\right) \simeq \mathbb{Z} \oplus \mathbb{Z}$ ), поэтому система

$$
x_{i}-\pi x_{i+1}=d\left(g_{i}, g_{i+1}\right),
$$

полученная из системы (1) критерия 1.3 при гомоморфизме sforg, разрешима в обратной последовательности из групп $H^{m-1}\left(\widetilde{X}, U_{i}\right)$. Другими словами, $h(\vartheta(\bar{f}))$ лежит в ядре sforg* ${ }^{*}$ т.е. в образе $\delta^{*}$.

Заметим, что по лемме 4.3 для разрешимости системы (1) достаточно (и необходимо), чтобы система (5) была разрешима в im sforg. Последнее вьполнено, в частности, если уже имеюшееся решение (4) лежит в imsforg, или, что то же самое, почленно отправляется в нуль гомоморфизмом alt. В любом случае, это решение переходит в нить, так как коэффициенты системы (5) идут в нули, поскольку они пришли из эквивариантных когомологий. Эта нить и является согласно определению связываюшего гомоморфизма одним из прообразов $h(\vartheta(\bar{f}))$ при $\delta^{*}$.

Остается заметить, что при гомоморфизме alt классы $\left(g_{i}^{2}\right)^{*}(\xi)$ переходят в $\left(g_{i}^{2}\right)_{\text {skew }}^{*}\left(\xi_{\text {skew }}\right)$, где $\xi_{\text {skew }}=\operatorname{alt} \xi$ (в случае $Q=\mathbb{R}^{m-1}$ это, очевидно, образующая групшы $\left.H_{\text {skew }}^{m-1}\left(S^{m-1}, S^{m-2}\right) \simeq \mathbb{Z}\right)$. Но по лемме $3.3,\left(\right.$ в) классы $\left(g_{i}^{2}\right)_{\text {skew }}^{*}\left(\xi_{\text {skew }}\right)$ и составляют нить $\check{\vartheta}(f)$.

Заметим теперь, что коэффициентные последовательности, задающие последовательности Смита, допускают эпиморфизмы на арифметические коэффициентные последовательности:

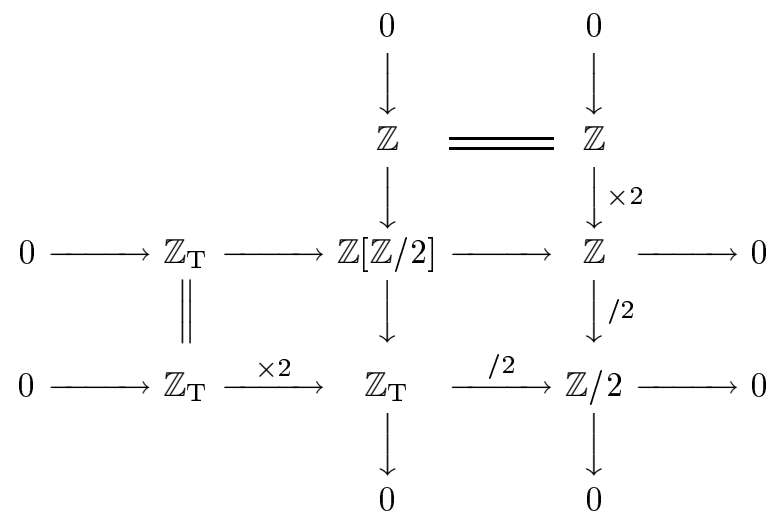

Это приводит, в частности, к гомоморфизму первой последовательности Смита на эквивариантную последовательность Бокштейна:

$$
\begin{aligned}
& \cdots \rightarrow H_{\mathrm{eq}}^{m}(K, L) \stackrel{\text { sforg }}{\longrightarrow} H^{m}(K, L) \stackrel{\text { alt }}{\longrightarrow} \quad H_{\text {skew }}^{m}(K, L) \quad \stackrel{\smile e}{\longrightarrow} H_{\text {skew }}^{m+1}(K, L) \rightarrow \cdots \\
& \cdots \rightarrow H_{\mathrm{eq}}^{m}(K, L) \stackrel{\times 2}{\longrightarrow} H_{\mathrm{eq}}^{m}(K, L) \stackrel{/ 2}{\longrightarrow} H^{m}(K / \mathfrak{t}, L / \mathfrak{t} ; \mathbb{Z} / 2) \stackrel{\beta}{\longrightarrow} H_{\text {skew }}^{m+1}(K, L) \rightarrow \cdots
\end{aligned}
$$

В частности, оказывается, что умножение на $k$-ю степень класса Эйлера является попросту композицией приведения по модулю 2 и $k$ гомоморфизмов Бокштейна $\beta^{k}$ (в альтернирующихся локальных коэффициентах). 
СлЕДСТвИЕ 4.5. В условиях наблюдения $4.4 \vartheta(\bar{f})=h_{2}^{-1} \delta_{2}^{*} \rho_{2}(\check{\vartheta}(f))$, әде $h_{2}-$ изоморфизм, аналогичный $h$ из леммы $4.3, \rho_{2}: \operatorname{ker} e \rightarrow \operatorname{ker} \beta$ - ограничение гомоморфизма приведения по модулю 2, а $\delta_{2}^{*}-$ связывающий гомоморфизм из точной последовательности

$$
0 \longrightarrow \lim _{\longleftarrow} A_{i}^{\prime} \longrightarrow \lim _{\longleftarrow} B_{i}^{\prime} \longrightarrow \lim _{\longleftarrow} C_{i}^{\prime} \stackrel{\delta_{2}^{*}}{\lim ^{1}} A_{i}^{\prime} \longrightarrow \lim ^{1} B_{i}^{\prime} \longrightarrow \lim ^{1} C_{i}^{\prime} \longrightarrow 0,
$$

построенной по короткой точной последовательности обратных последовательностей

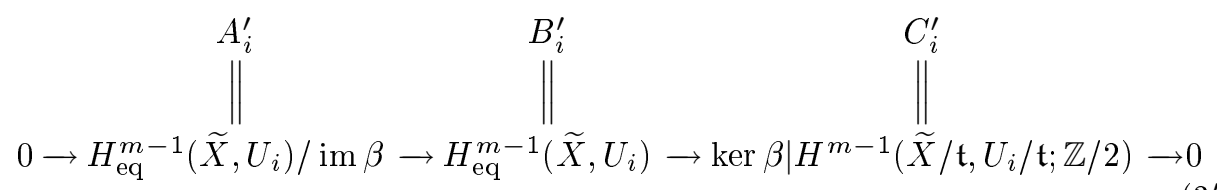

ДокАЗАТЕЛЬСТво. Достаточно сослаться на естественность связьваюшего гомоморфизма.

ПРЕДЛОЖЕНИЕ 4.6. Предположим, что композичия $\bar{f}: S^{n} \stackrel{f}{\longrightarrow} S^{n} \subset \mathbb{R}^{2 n}$, $n \geqslant 3$, реализуема дискретно, но не изотопически, и пусть $x \in S^{n}-$ любая точка, $P_{x}=f^{-1}(f(x))$. Пусть $O_{i} \subset \widetilde{S^{n}}-$ замыкание дополнения к инвариантной полиэдральной окрестности множества $S_{x}=\left(P_{x} \times\{x\} \cup\{x\} \times P_{x}\right) \backslash$ $\{(x, x)\}$, лежащей в его $2^{-i}$-окрестности и содержащей его $2^{-i-1}$-окрестность. Если каждый әлемент $\vartheta_{i}(f)$ нити ван Кампена-Скопенкова 乙-делится на класс Эйлера е $(\mathfrak{t})$, то группа $\lim ^{1} H_{\mathrm{eq}}^{2 n-1}\left(\widetilde{S^{n}}, O_{i}\right)$ содержит әлемент порядка 2.

ДОКАЗАТЕЛЬСТво. По условию каждый класс $\vartheta_{i}(f)=\alpha_{i} \smile e$ для некоторого $\alpha_{i} \in H_{\text {skew }}^{n-1}\left(\widetilde{S^{n}}, U_{i}\right)$. Следовательно, согласно лемме $3.3,\left(\right.$ a) $\vartheta_{i}(\widehat{f})=\alpha_{i} \smile e^{n}$, где $\widehat{f}$ обозначает композицию $f$ и включения $S^{n} \subset \mathbb{R}^{2 n-1}$. По лемме $4.1 e^{n}$ получен расширением носителя некоторого $E_{i} \in H_{\text {skew }}^{n}\left(\widetilde{S^{n}}, V_{i}\right)$, где $V_{i}-$ замькание дополнения к инвариантной полиэдральной окрестности множества $S=\{q\} \times$ $\left(S^{n} \backslash\{q\}\right) \cup\left(S^{n} \backslash\{q\}\right) \times\{q\}$, лежашей в его $2^{-i}$-окрестности, содержашей его $2^{-i-1}$-окрестность и распадающейся на две компоненты связности, переставляемые инволюцией $\mathfrak{t}$. Можно считать, что $O_{i}=V_{i} \cup U_{i}$, следовательно, класс $\vartheta_{i}(\widehat{f})$ получен расширением носителя из $\alpha_{i} \smile E_{i} \in H_{\mathrm{skew}}^{2 n-1}\left(\widetilde{S^{n}}, O_{i}\right)$. Более обшо, имеет место эпиморфизм $\varphi: K_{i} \rightarrow M_{i}$, где $K_{i}$ - образ умножения на $E_{i}$ в группе $H_{\text {skew }}^{2 n-1}\left(\widetilde{S^{n}}, O_{i}\right)$, а $M_{i}$ - образ умножения на $e^{n}$ в группе $H_{\text {skew }}^{2 n-1}\left(\widetilde{S^{n}}, U_{i}\right)$. Поскольку подгруппа $M_{i}$ имеет период 2 ввиду $2 e=0$, она отображается изоморфно на свой образ $N_{i}$ при приведении коэффициентов по модулю 2

$$
\rho_{2}: H_{\text {skew }}^{2 n-1}\left(\widetilde{S^{n}}, U_{i}\right) \rightarrow H^{2 n-1}\left(\widetilde{S^{n}} / \mathfrak{t}, U_{i} / \mathfrak{t} ; \mathbb{Z} / 2\right) .
$$

Эпиморфизм $\rho_{2} \varphi$ пропускается через образ $L_{i}$ группы $K_{i}$ при приведении коэффициентов по модулю 2

$$
H_{\text {skew }}^{2 n-1}\left(\widetilde{S^{n}}, O_{i}\right) \rightarrow H^{2 n-1}\left(\widetilde{S^{n}} / \mathfrak{t}, O_{i} / \mathfrak{t} ; \mathbb{Z} / 2\right)
$$


Пусть $\rho_{2} \varphi: K_{i} \stackrel{\psi}{\longrightarrow} L_{i} \stackrel{\chi}{\longrightarrow} N_{i}$ - соответствуюшее разложение, тогда классы $\psi\left(\alpha_{i} \smile E_{i}\right)$, не обязательно образующие нить, переходят в нить из классов $\rho_{2}\left(\vartheta_{i}(\widehat{f})\right)$ при эпиморфизме $\chi$. Так как ядро $\chi$ конечно, будучи подгруппой конечной группы $L_{i}$, из шестичленной последовательности, построенной по короткой точной

$$
0 \rightarrow \operatorname{ker} \chi \rightarrow L_{i} \stackrel{\chi}{\longrightarrow} N_{i} \rightarrow 0,
$$

вытекает, что существует нить $\left(\gamma_{i}\right) \in \underset{\lim }{\longleftarrow}\left(L_{i}\right)$ такая, что $\chi\left(\gamma_{i}\right)=\rho_{2}\left(\vartheta_{i}(\widehat{f})\right)$ для каждого $i$. С другой стороны, группа $K_{i}$ лежит в ядре умножения на класс Эйлера, так как его ограничение на дополнение к $O_{i}$ тривиально по построению $V_{i}$. Значит, ввиду эпиморфности $\psi$ группа $L_{i}$ лежит в ядре гомоморфизма Бокштейна. Тогда, поскольку $\vartheta(\vec{f}) \neq 0$, утверждение следует из следствия 4.5 и коммутативности диаграммы

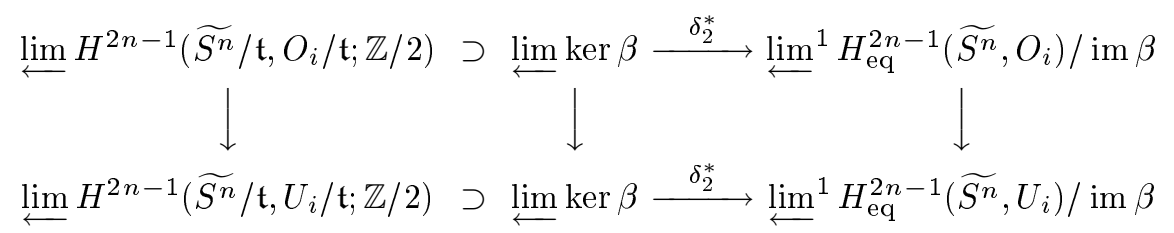

где $\delta^{*}$ из верхней строчки - связывающий гомоморфизм из шестичленной последовательности, построенной по короткой точной

$0 \rightarrow H_{\mathrm{eq}}^{2 n-1}\left(\widetilde{S^{n}}, O_{i}\right) / \operatorname{im} \beta \rightarrow H_{\mathrm{eq}}^{2 n-1}\left(\widetilde{S^{n}}, O_{i}\right) \rightarrow \operatorname{ker} \beta \mid H^{2 n-1}\left(\widetilde{S^{n}} / \mathfrak{t}, O_{i} / \mathfrak{t} ; \mathbb{Z} / 2\right) \rightarrow 0$.

\section{§5. Нерасщепимость на бесконечности}

Связывающий гомоморфизм $\delta^{*}$ из наблюдения 4.4 может быть описан алгебраически следующим образом. Заметим сначала, что обратный предел $\lim \mathscr{A}$, где $\mathscr{A}=\left\{A_{i}, \pi_{i}\right\}$, изоморфен группе $\operatorname{Hom}(\mathscr{Z}, \mathscr{A})$, где $\mathscr{Z}$ - обратная последовательность, каждый этаж которой $\mathbb{Z}$, а каждое отображение - тождество. Бифунктор Hom действует на категории $\mathfrak{S}$ обратных последовательностей абелевых групा (подробнее см. ниже), т.е. рассматриваются лишш такие наборы гомоморфизмов, которые коммутируют со спектральными проекциями. Но тогда в силу единственности первого производного функтора

$$
\lim ^{1} \mathscr{A} \simeq \operatorname{Ext}(\mathscr{Z}, \mathscr{A})
$$

где Ext - бифунктор, производньй от Нот по каждому аргументу и изучавшийся в [40]. Стандартные рассуждения показьвают, что группа $\operatorname{Ext}(\mathscr{C}, \mathscr{A})$ состоит из классов эквивалентности расширений $0 \rightarrow \mathscr{A} \rightarrow \mathscr{E} \rightarrow \mathscr{C} \rightarrow 0$ (здесь все гомоморфизмы - из категории S) с обычным сложением Бэра (см. [21]). Явная конструкция изоморфизма между производным пределом (определенным как в §1) и группой расширений доставляется описанием расширений в терминах бесконечных систем уравнений на гомоморфизмы, которое приводится ниже. Из него, в частности, ясно, что сопоставление короткой точной последовательности $0 \rightarrow \mathscr{A} \rightarrow \mathscr{B} \rightarrow \mathscr{C} \rightarrow 0$ связьваюшего гомоморфизма $\delta^{*}: \lim _{\longleftarrow} \mathscr{C} \rightarrow \lim ^{1} \mathscr{A}$ есть не что иное, как произведение Йонеды (см. [21])

$$
\operatorname{Hom}(\mathscr{Z}, \mathscr{C}) \otimes \operatorname{Ext}(\mathscr{C}, \mathscr{A}) \rightarrow \operatorname{Ext}(\mathscr{Z}, \mathscr{A}) .
$$


Для изучения групшы расширений нам понадобится небольшая категорная подготовка.

Соглашения. Из соображений простоты терминологии далее под спектром понимается исключительно обратная последовательность (т.е. обратный спектр, индексированный натуральньми числами) абелевых групп. Мы называем спектр $\mathscr{Y}=\left\{Y_{i}, \pi_{i}^{\prime}\right\}$ подспектром спектра $\mathscr{X}=\left\{X_{i}, \pi_{i}\right\}$, если каждый $Y_{i} \subset X_{i}$ и каждая $\pi_{i}^{\prime}=\left.\pi_{i}\right|_{Y_{i+1}}$.

Категории диаграмм. Спектры образуют категорию $\mathfrak{S}$, морфизмами которой являются коммутативные диаграммы

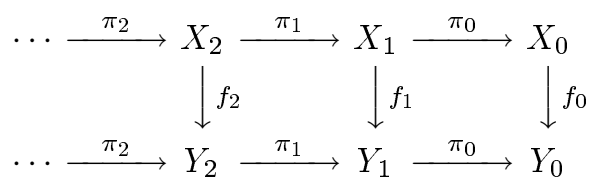

обозначаемые $f: \mathscr{X} \rightarrow \mathscr{Y}$. Это частный случай категории диаграмм Гротендика, объектами которой являются функторы из (индексируюшей) малой категории $\mathfrak{I}$ в (индексируемую) абелеву категорию $\mathfrak{A}$, а морфизмами - их естественные преобразования; в данном случае $\mathfrak{A}$ - категория абелевых груп, а $\mathfrak{I}$ - категория, объектами которой являются натуральные числа, а морфизмами - стрелки $m \rightarrow n$ при условии $m \geqslant n$.

Нам понадобятся еще три категории диаграмм. Взяв вместо $\mathfrak{I}$ категорию $\mathfrak{I}^{\prime}$, объектами которой являются натуральные числа, а морфизмы лишь тождественные, в качестве категории диаграмм получим категорию $\mathfrak{S}^{\prime}$ упорядоченных счетных наборов абелевых групп; функтор включения $\mathfrak{I}^{\prime} \rightarrow \mathfrak{I}$ доставляет функтор забывания стрелок $\square: \mathfrak{S} \rightarrow \mathfrak{S}^{\prime}$. Этот функтор пропускается через $\bowtie: \mathfrak{S} \rightarrow \mathfrak{S}^{\prime \prime}$, где $\mathfrak{S}^{\prime \prime}$ - "допредельная" категория, индексируемая категорией $\mathfrak{I}^{\prime \prime}$, объектами которой являются упорядоченные пары $(m, n)$ натуральных чисел, где $m \leqslant n$, а морфизмами - стрелки $(m, n) \rightarrow\left(m^{\prime}, n\right)$ при условии $m^{\prime} \leqslant m$. Функтор $\bowtie$ можно считать диагональньм произведением функторов "отрезания бесконечного хвоста" $\left\lceil n: \mathfrak{S} \rightarrow \mathfrak{S}_{n}\right.$, где $\mathfrak{S}_{n}-$ категория диаграмм, индексированных ограничением $\mathfrak{I}_{n}$ категории $\mathfrak{I}$ на множество объектов $\{0, \ldots, n\}$.

Функтор Ext $\square$. Пусть $\mathscr{X}=\left\{X_{i}, \pi_{i}\right\}, \mathscr{Y}=\left\{X_{i}, \pi_{i}\right\}$ - спектры. Бифунктор $\operatorname{Hom}(\mathscr{X}, \mathscr{Y})$ из категории спектров в категорию абелевых групп есть по определению ядро гомоморфизма $\varphi: \prod \operatorname{Hom}\left(X_{i}, Y_{i}\right) \rightarrow \prod \operatorname{Hom}\left(X_{i}, Y_{i-1}\right)$, отправляюшего каждьй набор $\alpha$ гомоморфизмов $\alpha_{i}: X_{i} \rightarrow Y_{i}$ в набор $\alpha_{i} \pi_{i}-\pi_{i} \alpha_{i+1}$, измеряюший отклонение от коммутативности наборов гомоморфизмов $\alpha$ и $\pi_{i}$. Мы утверждаем, что коядро этого гомоморфизма изоморфно группе $\operatorname{Ext}_{\square}(\mathscr{X}, \mathscr{Y})$, где $\operatorname{Ext}_{\square}-$ производный функтор функтора Ноm (скажем, по второму аргументу) в относительной категории (см., например, [21]), задаваемой функтором $\square$ забывания спектральных проекций:

$$
0 \rightarrow \operatorname{Hom}(\mathscr{X}, \mathscr{Y}) \rightarrow \prod_{i} \operatorname{Hom}\left(X_{i}, Y_{i}\right) \stackrel{\varphi}{\longrightarrow} \prod_{i} \operatorname{Hom}\left(X_{i}, Y_{i-1}\right) \rightarrow \operatorname{Ext}_{\square}(\mathscr{X}, \mathscr{Y}) \rightarrow 0
$$

В самом деле, стандартными рассуждениями показывается, что Ext $\square$ сопоставляет спектрам $\mathscr{X}, \mathscr{Y}$ подгруппу $\square$-расшепляюшихся расширений в группе $\operatorname{Ext}(\mathscr{X}, \mathscr{Y})$ 
всех расширений $0 \rightarrow \mathscr{Y} \rightarrow \mathscr{E} \rightarrow \mathscr{X} \rightarrow 0$. Подчеркнем, что $\square$-расшепляющаяся короткая точная последовательность спектров - это такая последовательность, каждый этаж которой расщепляется как короткая точная последовательность абелевых групп независимо от остальных этажей. Но тогда легко видеть, что по отношению к функтору $\operatorname{Ext}_{\square}(\mathscr{X}, *)$ (и высшим $\square$-производным функторам от Нот, которые тривиальны ввиду существования двучленных резольвент у абелевых групп) ациклична известная резольвента Рооса

$$
\stackrel{\varepsilon}{\longrightarrow}\left\{\prod_{j=0}^{i} Y_{j} ; \xi_{i}\right\} \stackrel{\psi}{\longrightarrow}\left\{\prod_{j=0}^{i-1} Y_{j} ; \xi_{i}\right\} \rightarrow 0
$$

обратного спектра У , где $\xi_{i}$ - декартовы проекции прямых произведений на сомножители, аугментация $\varepsilon$ задается диагональными отображениями $Y_{i} \rightarrow \prod_{j=0}^{i} Y_{j}$, $y \mapsto\left(\ldots, \pi_{i-2} \circ \pi_{i-1}(y), \pi_{i-1}(y), y\right)$, а гомоморфизм $\psi$ определяется формулой $\left(y_{0}, \ldots, y_{i}\right) \mapsto\left(y_{0}-\pi_{1}\left(y_{1}\right), \ldots, y_{i-1}-\pi_{i}\left(y_{i}\right)\right)$. Кроме того, резольвента Рооса, очевидно, $\square$-расшепляется, поэтому группа coker $\varphi$, будучи группой одномерных гомологий коцепного комплекса, полученного применением функтора $\operatorname{Hom}(\mathscr{X}, *)$ к резольвенте Рооса, изоморфна $\operatorname{Ext}_{\square}(\mathscr{X}, \mathscr{Y})$. Функтор $\operatorname{Ext}_{\square}$ аналогичен функтоpy "Рехt" сервантных расширений в теории абелевых групп (см. [41], ср. [31]).

Полное препятствие к расщепимости при условии $\square$-расщепимости. Пусть $\mathscr{S}: 0 \rightarrow \mathscr{A} \stackrel{\mathrm{i}}{\rightarrow} \mathscr{B} \stackrel{\mathrm{j}}{\rightarrow} \mathscr{C} \rightarrow 0$ - короткая точная последовательность спектров

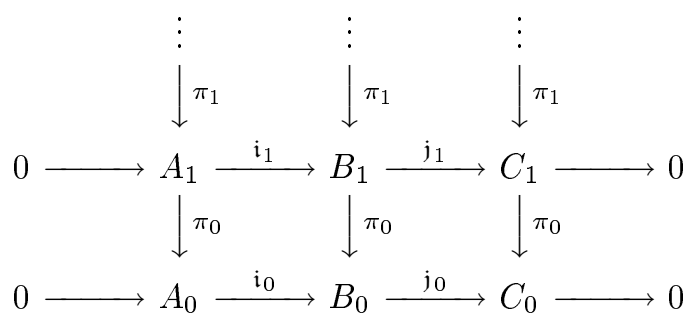

Если $\mathscr{S} \square$-расщепима, имеется набор гомоморфизмов $s_{i}: C_{i} \rightarrow B_{i}$ таких, что $\mathfrak{j}_{i} \circ s_{i}=1$. Гомоморфизмы $s_{i} \in \operatorname{Hom}\left(C_{i}, B_{i}\right)$ можно заменить на некоторые $r_{i}$, коммутирующие со спектральными проекциями, если и только если найдутся $x_{i} \in$ $\operatorname{Hom}\left(C_{i}, A_{i}\right)$ такие, что

$$
x_{i} \circ \pi_{i}-\pi_{i} \circ x_{i+1}=d_{i}
$$

где $d_{i}=s_{i} \circ \pi_{i}-\pi_{i} \circ s_{i+1} \in \operatorname{Hom}\left(C_{i}, \mathfrak{i}_{i}\left(A_{i}\right)\right)$. В самом деле, если $x_{i}$ нашлись, подходят $r_{i}=s_{i}-x_{i}$, а если $r_{i}$ уже имеются, в качестве $x_{i}$ можно взять $s_{i}-r_{i}$. Таким образом, последовательность $\mathscr{S}$ не расщепляется, если и только если бесконечньй набор гомоморфизмов $\left(s_{i} \circ \pi_{i}-\pi_{i} \circ s_{i+1}\right) \in \prod_{i} \operatorname{Hom}\left(C_{i}, A_{i-1}\right)$ задает неразрешимую систему уравнений

$$
[\mathscr{S}] \in \operatorname{Ext}_{\square}(\mathscr{C}, \mathscr{A})
$$


Функтор $\operatorname{Ext}_{\bowtie}$. Обычным образом устанавливается, что группа $\operatorname{Ext}_{\bowtie}(\mathscr{X}, \mathscr{Y})$, где $\mathrm{Ext}_{\bowtie}-$ производный функтор от Нот в относительной категории, задаваемой функтором $\bowtie$ ограничения на конечные хвосты, изоморфна подгруппе всех $\bowtie$-расщепляюшихся расширений в группе $\operatorname{Ext}_{\square}(\mathscr{X}, \mathscr{Y}) \subset \operatorname{Ext}(\mathscr{X}, \mathscr{Y})$, т.е. расширений, первые $n$ этажей которых можно расщепить согласованно со спектральными проекциями отдельно для каждого натурального $n$. Следовательно, имеется короткая точная последовательность

$$
0 \rightarrow \operatorname{Ext}_{\bowtie}(\mathscr{X}, \mathscr{Y}) \rightarrow \operatorname{Ext}_{\square}(\mathscr{X}, \mathscr{Y}) \rightarrow \lim _{\longleftarrow} \operatorname{Ext}_{\square}(\mathscr{X}\lceil n, \mathscr{Y} \uparrow n) \rightarrow 0,
$$

позволяющая использовать резольвенту Рооса для вычисления $\mathrm{Ext}_{\bowtie}$. Отметим, что $\operatorname{Ext}_{\bowtie} \neq \mathrm{Ext}_{\square}$, как показьвают примеры

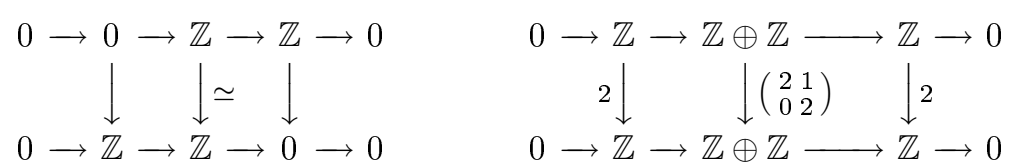

Лемма 5.1. (а) Если каждая спектральная проекция в $\mathscr{C}$ - мономорфизм, образ которого вицделяется прямым слагаемым, $\operatorname{Ext}_{\bowtie}(\mathscr{C}, \mathscr{A})=\operatorname{Ext}_{\square}(\mathscr{C}, \mathscr{A})$.

(б) Если один из спектров $\mathscr{A}, \mathscr{C}$ состоит из конечных групп, а другой из конечно порожденных, $\operatorname{Ext}_{\bowtie}(\mathscr{C}, \mathscr{A})=0$.

ДокаЗАТЕЛЬСтво. (а) Требуется построить решение бесконечной системы (6) на сколь угодно длинном конечном интервале $0, \ldots, N$. Выберем $x_{N+1}$ произвольньм образом. Предполагая, что $x_{i+1}$ уже найдено, положим

$$
x_{i}=\left(d_{i}-\pi_{i} \circ x_{i+1}\right) \circ \rho_{i},
$$

где $\rho_{i}: C_{i} \rightarrow C_{i+1}$ - расшепление спектральной проекции $\pi_{i}$.

ЗАмечаниЕ. В случае $\mathscr{C}=\mathscr{Z}$ стандартное доказательство (б) (например, в [42]) состоит в индуктивном распространении решения на верхние этажи и подходит для любого спектра $\mathscr{A}$, в котором выполнено условие Миттаг-Леффиера. В общем случае применить это рассуждение напрямую не удается, и мы ограничимся рассмотрением спектра из конечных групп.

(б) Предполагая, что неизвестные $x_{i}$ найдены при $i \leqslant n$, причем среди допредельных решений $x(\nu)=\left\{x_{i}(\nu) \mid i \leqslant \nu\right\}$, доставляемых $\bowtie$-расшепимостью, бесконечно много решений $x\left(\nu_{n}(i)\right), i=1,2, \ldots$, совпадающих с построенным на отрезке $1, \ldots, n$, заметим, что в конечной групше $\operatorname{Hom}\left(C_{n}, A_{n}\right)$ найдется хотя бы один элемент $x_{n+1}$, совпадающий с $x_{n+1}\left(\nu_{n}(i)\right)$ для некоторой бесконечной последовательности $i_{1}, i_{2}, \ldots$ индексов $i$. Для завершения шага индукции остается положить $\nu_{n+1}(j)=\nu_{n}\left(i_{j}\right)$.

Основным результатом этого параграфа является

Альтернатива 5.2. Предположим, что композиция $f: X \rightarrow \mathbb{R}^{2 n-1} u$ включения $\mathbb{R}^{2 n-1} \subset \mathbb{R}^{2 n}$ реализуема дискретно, но не изотопически, $n \geqslant 3$. Либо нить ван Кампена-Скопенкова $\check{\vartheta}(f) \in \lim H_{\text {skew }}^{2 n-1}\left(\widetilde{X}, U_{i}\right)$ является әле-

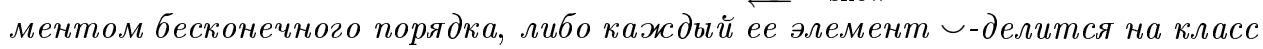
Эйлера $е(\mathfrak{t})$. 
ЗАМЕЧАНИЕ. Поскольку функтор обратного предела не точен, во втором случае нить $\check{\vartheta}(f)$, вообше говоря, может не получаться умножением на класс Эйлера ни из какой нити $(2 n-2)$-когомологий (например, если гомоморфизм умножения на класс Эйлера вьглядит как приведение по модулю 2 спектра из умножений на 3 в группе $\mathbb{Z})$.

ДОКАЗАТЕЛЬСТВО АЛЬТЕРНАТИВЫ 5.2. Как мы Выяснили при доказательстве наблюдения 4.4 , для разрешимости системы (1) достаточно, чтобы система (5) была разрешима в imsforg. Это выполнено, если подспектр imsforg выделяется прямьм слагаемьм (согласованно со спектральньми проекциями) в спектре из $H^{2 n-1}\left(\widetilde{X}, U_{i}\right)$ или даже в каком-нибудь его подспектре, содержашем решение (4) системы (5), так как в этом случае последнее можно спроектировать в imsforg (с категорной точки зрения расщепление короткой точной последовательности (3) гарантирует тривиальность связывающего гомоморфизма $\delta^{*}: \lim C_{i} \rightarrow \lim ^{1} A_{i}$, так как функтор обратного предела сохраняет точность расшепляющихся последовательностей). Для этого, в частности, необходимо $\square$-расщепление, т.е. выделение прямым слагаемым на каждом этаже спектра, равносильное (ввиду конечной порожденности групп спектра) сервантности ${ }^{6}$ каждой подгрупшы sforg $H_{\mathrm{eq}}^{2 n-1}\left(\widetilde{X}, U_{i}\right)$ в соответствующей группе.

Продолжению доказательства предпошлем два замечания.

ЗАмЕчАниЕ (в доказательстве не используется). Полезно иметь в виду следуюшее обстоятельство. Инволюция $\mathfrak{t}$ индуцирует на группе $M=H^{2 n-1}\left(\widetilde{X}, U_{i}\right)$ структуру $\mathbb{Z}[\mathbb{Z} / 2]$-модуля. "Симметричные" (инвариантные) элементы $M^{\mathbb{Z} / 2=}$ $H^{0}(\mathbb{Z} / 2 ; M)$ составляют в точности ядро композиции aforg o alt: $M \rightarrow M$, поэтому alt-образ элемента $m \in M$ делится на класс Эйлера, если и только если $m$ "симметричньй", но не лежит в образе гомоморфизма sforg. C другой стороны, если $m$ лежит в образе sforg, но не является “симметризацией", т.е. не лежит в образе композиции sforg o sym: $M \rightarrow M$, то любой sforg-прообраз $m$ выживает при умножении на класс Эйлера. Таким образом, элемент $m$ можно “сдвинуть” в соседнюю размерность с помощью первой последовательности Смита, если и только если он "симметричен”, но не является “симметризацией”, представляя тем самьм ненулевой элемент группы $H_{1}(\mathbb{Z} / 2 ; M)$. Аналогично, $m$ можно “сдвинуть” в соседнюю размерность с помощью второй последовательности Смита, если и только если он “кососимметричен”, но не является “альтернацией”, представляя ненулевой элемент группы $H^{1}(\mathbb{Z} / 2 ; M)$.

ЗАмЕчАниЕ (в доказательстве не используется). Нерасщепимость первой последовательности Смита в члене $H^{2 n-1}\left(\widetilde{X}, U_{i}\right)$ сама по себе еще не гарантирует нетривиальности умножения на класс Эйлера в члене $H_{\mathrm{skew}}^{2 n-2}\left(\widetilde{X}, U_{i}\right)$ второй последовательности Смита. В самом деле, сушествует ацикличный (в смысле равенства нулю гомологий и когомологий ненулевой размерности) $\mathbb{Z}[\mathbb{Z} / 2]$-модуль, в котором инвариантная подгруппа не выделяется прямьм слагаемьм:

$$
\mathbb{Z} \oplus \mathbb{Z} \oplus \mathbb{Z} / 2=\langle x\rangle \oplus\langle y\rangle \oplus\langle z \mid 2 z=0\rangle, \quad x \mapsto x+z, \quad y \mapsto-y+z, \quad z \mapsto z .
$$

Значит, последовательности Смита в некоторой размерности могут иметь вид

$$
0 \underset{\smile e}{\stackrel{\smile e}{\leftrightarrows}} \mathbb{Z} \oplus \mathbb{Z} / 2 \underset{\text { sforg }}{\stackrel{\text { sym }}{\leftrightarrows}} \mathbb{Z} \oplus \mathbb{Z} \oplus \mathbb{Z} / 2 \underset{\text { alt }}{\stackrel{\text { aforg }}{\leftrightarrows}} \mathbb{Z} \oplus \mathbb{Z} / 2 \underset{\smile e}{\stackrel{\longleftarrow}{\leftrightarrows}} 0,
$$

\footnotetext{
${ }^{6}$ Напомним, что сервантность подгруппы $H$ группы $G$ означает делимость любого $h \in H$ на любое натуральное $n$ внутри подгруппы $H$ при условии делимости $h$ на $n$ с выходом в $G$.
} 
и, тем самым, альтернативу 5.2 не удастся доказать, исходя только из них.

ПРОДОЛЖЕНИЕ ДОКАЗАТЕЛЬСТВА АЛЬТЕРНАТИВЫ 5.2. ЗаметИМ, что система (5) в действительности разрешима в подспектре $\mathscr{S}$, состояшем на каждом $i$-м этаже из внутренней суммы $S_{i}$ образов гомоморфизмов

$$
\widetilde{g}_{j}^{*}: \mathbb{Z} \oplus \mathbb{Z}=H^{2 n-1}\left(S^{2 n-1}, S^{2 n-2}\right) \rightarrow H^{2 n-1}\left(\widetilde{X}, U_{i}\right)
$$

для всех $j \geqslant i$. Так как каждое $\widetilde{g}_{i}$ эквивариантно, каждый гомоморфизм $\widetilde{g}_{i}^{*}$ модульньй (относительно инволюций $\mathfrak{s}^{*}$ и $\mathfrak{t}^{*}$, задающих структуры $\mathbb{Z}[\mathbb{Z} / 2]$-модулей), причем взятие обратного образа коммутирует с альтернацией alt, a также с забываниями aforg и sforg.

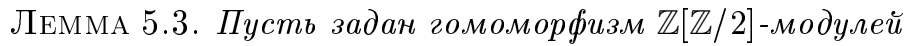

$$
\varphi:\left(H^{2 n-1}\left(S^{2 n-1}, S^{2 n-2}\right), \mathfrak{s}^{*}\right) \rightarrow\left(H^{2 n-1}\left(\widetilde{X}, U_{i}\right), \mathfrak{t}^{*}\right),
$$

а также групповые гомоморфизмы

$$
\begin{aligned}
\varphi_{\mathrm{eq}}: H_{\mathrm{eq}}^{2 n-1}\left(S^{2 n-1}, S^{2 n-2}\right) & \rightarrow H_{\mathrm{eq}}^{2 n-1}\left(\widetilde{X}, U_{i}\right), \\
\varphi_{\text {skew }}: H_{\text {skew }}^{2 n-1}\left(S^{2 n-1}, S^{2 n-2}\right) & \rightarrow H_{\text {skew }}^{2 n-1}\left(\widetilde{X}, U_{i}\right),
\end{aligned}
$$

причем следующие четыре квадрата (с одноименными горизонтальными стрелками) коммутативны:

$$
\begin{aligned}
& H_{\mathrm{eq}}^{2 n-1}\left(S^{2 n-1}, S^{2 n-2}\right) \underset{\text { sforg }}{\stackrel{\mathrm{sym}}{\leftrightarrows}} H^{2 n-1}\left(S^{2 n-1}, S^{2 n-2}\right) \underset{\text { alt }}{\stackrel{\text { aforg }}{\leftrightarrows}} H_{\text {skew }}^{2 n-1}\left(S^{2 n-1}, S^{2 n-2}\right) \\
& \varphi_{\text {eq }} \downarrow \quad \varphi \downarrow \quad \varphi_{\text {skew }} \downarrow \\
& H_{\mathrm{eq}}^{2 n-1}\left(\widetilde{X}, U_{i}\right) \quad \underset{\text { sforg }}{\stackrel{\text { sym }}{\leftrightarrows}} H^{2 n-1}\left(\widetilde{X}, U_{i}\right) \quad \underset{\text { alt }}{\leftrightarrows} \quad H_{\text {skew }}^{2 n-1}\left(\widetilde{X}, U_{i}\right)
\end{aligned}
$$

Если $\varphi_{\mathrm{eq}}-$ мономорфизм, $\alpha \in \operatorname{im} \varphi$, и некоторое кратное $\alpha$, но не он сам, лежит в imsforg $\backslash\{0\}$, то alt $(\alpha)$ делится на класс Эйлера $е(\mathfrak{t})$.

ДокАЗАТЕЛЬСтво. Если $m \alpha \in \mathrm{im} \operatorname{sforg}$ для некоторого натурального $m$, то $2 m \alpha \in K:=\operatorname{im}(\varphi \circ$ sforg), как видно из п. (i) замечания (см. $\S 4)$. Пусть $\beta \in K-$ какой-нибудь элемент, не являющийся кратньм никакого элемента из $K$ и такой, что $2 m \alpha=m^{\prime} \beta$ для некоторого натурального $m^{\prime}$. Пусть $b=2 m /\left(2 m, m^{\prime}\right)$ и $a=m^{\prime} /\left(2 m, m^{\prime}\right)$, тогда $b \alpha=a \beta$ и найдутся $p, q \in \mathbb{Z}$ такие, что $p a+q b=1$. Тогда $\gamma:=p \alpha+q \beta \in \operatorname{im} \varphi$ обладает свойствами $a \gamma=\alpha-$ откуда $\gamma \notin \operatorname{imsforg}$ и тем более $\gamma \notin K-$ и $b \gamma=\beta \in K$, причем $\langle b \gamma\rangle$ сервантна в $K$.

Пусть $x$ и $y$ обозначают $\varphi(\xi)$ и $\varphi(\mathfrak{t} \xi)$ соответственно, где $\xi$ - какая-нибудь образующая, тогда $\gamma=k x+l y$ для некоторых $k, l \in \mathbb{Z}$, a $K=\langle x+y\rangle$. Значит, $b(k x+l y)=x+y$, т.е. $(b k-1) x=(1-b l) y$. Поскольку $\mathfrak{t}^{*} \varphi=\varphi \mathfrak{t}^{*}$, должно быть выполнено и $(1-b l) x=(b k-1) y$. Вычитая это равенство из предыдушего, получаем $(b k-2+b l)(x+y)=0$, но так как $\varphi_{\text {еq }}-$ мономорфизм, это означает, что $b(k+l)=2$, т.е. $b=2$ и $k+l=1$. Положим $r=k-l$, тогда $2(k x+l y)=x+y$ перепишется как $r(x-y)=0$, причем $r=2 k-1$ нечетно.

Предположим, что имеет место равенство $p x=q y$, где $p, q \in \mathbb{Z}$. Снова используя симметрию $\mathfrak{t}^{*} \varphi=\varphi \mathfrak{t}^{*}$, находим $(p-q)(x+y)=0$, что в силу инъективности $\varphi$ еq 
означает $p=q$. Следовательно, циклический $\mathbb{Z}[\mathbb{Z} / 2]$-модуль $\operatorname{im} \varphi$ получен из кольца $\mathbb{Z}[\mathbb{Z} / 2]$ факторизацией по идеалу, порожденному несколькими элементами вида $p_{i}(\xi-\mathfrak{t} \xi)$, или, что то же самое, одним элементом $p(\xi-\mathfrak{t} \xi)$, где $p$ - наибольший общий делитель всех $p_{i}$. Поскольку тем самьм $p \mid r$, это $p$ нечетно. Если $p=1$, образ относительных когомологии сферы

$$
\mathbb{Z} \underset{\text { sforg }}{\stackrel{\text { sym }}{\leftrightarrows}} \mathbb{Z} \oplus \mathbb{Z} \underset{\text { alt }}{\stackrel{\text { aforg }}{\leftrightarrows}} \mathbb{Z}
$$

при гомоморфизме $\varphi$ приобретает вид абсолютных

$$
\mathbb{Z} \underset{\text { sforg }}{\stackrel{\text { sym }}{\leftrightarrows}} \underset{\text { alt }}{\stackrel{\text { aforg }}{\leftrightarrows}} \mathbb{Z} / 2
$$

Поскольку $\alpha$ не лежит в im sforg, но попадает туда при умножении на некоторое $m$, элемент $\operatorname{alt}(\alpha) \in \mathbb{Z} / 2$ нетривиален. Если $p>1$, то $K=\operatorname{im} \operatorname{sforg} \cap \operatorname{im} \varphi$ в силу нечетности $p$, поэтомy alt $(\operatorname{im} \varphi)=\operatorname{im} \varphi_{\text {skew }}$ есть циклическая группа порядка $2 p$ :

$$
\mathbb{Z} \underset{\text { sforg }}{\stackrel{\text { sym }}{\leftrightarrows}} \mathbb{Z} \oplus \mathbb{Z} / p \underset{\text { alt }}{\stackrel{\text { aforg }}{\leftrightarrows}} \mathbb{Z} / 2 \oplus \mathbb{Z} / p
$$

Так как $\alpha$ не лежит в $K$, но попадает туда при умножении на $2, \operatorname{alt}(\alpha)$ есть единственный элемент порядка 2 в этой группе.

Наконец, поскольку в іт $\varphi$ элементы порядка 2 отсутствуют в обоих случаях, aforg $\operatorname{alt}(\alpha)=0$. Значит, в силу точности второй последовательности Смита $\operatorname{alt}(\alpha)$ делится на класс Эйлера.

ОКОНЧАНИЕ ДОКАЗАТЕЛЬСТВА АЛЬТЕРНАТИВЫ 5.2. Подспектр $\mathscr{S}$ содержится в подспектре alt $^{-1}(\mathscr{T})$ спектра из групп $H^{2 n-1}\left(\widetilde{X}, U_{i}\right)$, где $\mathscr{T}$ состоит из циклических подгрупп $T_{i}$, порожденных элементами $\vartheta_{i}(f)=\left(\widetilde{g}_{i}\right)_{\text {skew }}^{*}\left(\xi_{\text {skew }}\right)$ нити $\check{\vartheta}(f) \in \lim H_{\text {skew }}^{2 n-1}\left(\widetilde{X}, U_{i}\right)$. Предположим, что нить $\check{\vartheta}(f)$ - элемент конечного порядка. Тогда, как следует из леммы 5.1, если короткая точная последовательность спектров

$$
0 \rightarrow H_{\mathrm{eq}}^{2 n-1}\left(\widetilde{X}, U_{i}\right) / \text { ime } \rightarrow \operatorname{alt}^{-1}(\mathscr{T}) \rightarrow \mathscr{T} \rightarrow 0
$$

оказалась $\square$-расшепимой, она расщепляется (в категории спектров $\mathfrak{S}$ ), и решение (4) можно спроектировать в эквивариантный спектр, получив, что $f$ изотопически реализуемо. Если же $\square$-расщепимость отсутствует при ограничении спектра на сколь угодно далекие бесконечные хвосты, подспектр im sforg на некоторых сколь угодно высоких этажах не сервантен в соответствующих этажах спектра $\operatorname{alt}^{-1}(\mathscr{T})=(\mathrm{im} s f o r g) \mathscr{S}$. Но тогда найдутся и сколь угодно высокие этажи подспектра $\mathscr{S} \cap \mathrm{imsforg}$ не сервантные в своих этажах спектра $\mathscr{S}$. Значит, в подгрупше $S_{i}$ групшы $H^{2 n-1}\left(\widetilde{X}, U_{i}\right)$ найдется элемент $\alpha$ такой, что $m \alpha \in \operatorname{im}$ sforg для некоторого натурального $m$, хотя сам $\alpha \notin \mathrm{im}$ sforg. Образ alt $\alpha \in T_{i}$ является некоторым кратным $\vartheta_{i}(f)$, следовательно, без ограничения общности совпадает с $\vartheta_{i}(f)$ в силу эпиморфности alt: $S_{i} \rightarrow T_{i}$. По определению подгрупшы $S_{i} \alpha=\alpha_{1}+\cdots+\alpha_{r}$, где каждый $\alpha_{j}$ лежит в образе $\widetilde{g}_{l_{j}}^{*}$ для некоторого $l_{j} \geqslant i$. Подправляя каждый $\alpha_{j}$ на некоторый элемент образа композиции $\widetilde{g}_{l_{j}}^{*} \circ$ sforg, без ограничения общности можно считать, что каждый $\alpha_{j}$ является прообразом (при соответствуюшем ему гомоморфизме $\widetilde{g}_{l_{j}}^{*}$ ) некоторого кратного $m_{j} \xi$ выбранной образующей $\xi$. Остается применить лемму 5.3 к модульному гомоморфизму $\varphi=m_{1} \widetilde{g}_{l_{1}}^{*}+\cdots+m_{r} \widetilde{g}_{l_{r}}^{*}$. 
ЗАмечАнИЕ. Случай $p>1$ в доказательстве леммы 5.3 в действительности не реализуется ни для какого $\alpha$, переходящего в $\vartheta_{i}(f)$ при гомоморфизме alt, поскольку $\vartheta_{i}(f)$ является прообразом образующей при каждом из гомоморфизмов $\left(\widetilde{g}_{l_{i}}\right)_{\mathrm{skew}}^{*}$, а значит, и при их линейной комбинации $\varphi_{\text {skew }}$.

ЗАмЕчАниЕ. Пример 2 реализует возможность, предполагающую конечность порядка нити ван Кампена-Скопенкова. Что касается случая бесконечного порядка, он реализуется, по крайней мере, с алгебраической точки зрения (ср. [41; 29 , упр. 7]). Рассмотрим спектр

$$
\cdots \longrightarrow \mathbb{Z} \oplus \mathbb{Z} \stackrel{\left(\begin{array}{ll}
2 & 1 \\
1 & 2
\end{array}\right)}{\longrightarrow} \mathbb{Z} \oplus \mathbb{Z} \stackrel{\left(\begin{array}{ll}
2 & 1 \\
1 & 2
\end{array}\right)}{\longrightarrow} \mathbb{Z} \oplus \mathbb{Z}
$$

Перестановка слагаемых задает на каждом этаже структуру свободного циклического $\mathbb{Z}[\mathbb{Z} / 2]$-модуля, являющегося, разумеется, ацикличньм. Подспектр, состоящий из инвариантных подгрупп $\langle(x, x)\rangle$, имеет вид

$$
\stackrel{\times 3}{\longrightarrow} \mathbb{Z} \stackrel{\times 3}{\longrightarrow} \mathbb{Z}
$$

и сервантен на каждом этаже, но система $x_{i}-\pi x_{i+1}=(1,1)$, в нем не разрешимая, становится разрешимой во всем спектре: достаточно взять $x_{i}=(1,-2){ }^{7}$

ЗАмечАниЕ. Спектр из $\mathbb{Z}[\mathbb{Z} / 2]-$ модулей, обсуждавшийся в предшествующем замечании, допускает вложение, расшепляюшееся согласованно по этажам, в спектр из одномерных гомологий некоторого спектра из $\mathbb{Z} / 2$-графов (т.е. одномерных полиэдров со свободной инволюцией). А именно рассмотрим надстройку над 4-элементным множеством $S^{0} \times S^{0}$ с инволюцией $\sigma=\tau *\left(\tau \times \mathrm{id}_{S^{0}}\right)$, где $\tau$-свободная инволюция на $S^{0}$. Спектральная проекция $p: E\left(S^{0} \times S^{0}\right) \rightarrow E\left(S^{0} \times S^{0}\right)$ дается пережиманием надстройки в двух горизонтальных срезах, так что получаются три надстройки; далее верхняя и нижняя отображаются в $E\left(S^{0} \times S^{0}\right)$ тождеством, а центральная - инволюцией $\sigma$; легко видеть, что проекция $p$ эквивариантна относительно $\sigma$. Мы видим, что одномерные гомологии полученного спектра содержат по одной дополнительной образующей на каждом этаже по сравнению с рассмотренным ранее спектром. Это неслучайно, так как несложно показать, что невозможно найти точную геометрическую модель для последнего, не добавляя к нему дополнительных образующих.

\section{§6. Отображение, пропущенное через коразмерность $k$}

Далее будет доказано обобщение альтернативы 5.2 на случай отображений с образом в подмногообразии с тривиальньм нормальным расслоением. Это завершает доказательство когомологической версии теоремы $2,(\mathrm{a})$, начатое в предложении 4.6.

\footnotetext{
${ }^{7}$ Интересно, что приведенный спектр из $\mathbb{Z}[\mathbb{Z} / 2]$-модулей допускает вложение в спектр из свободных $\mathbb{Z}[\mathbb{Z} / 2]$-модулей, в котором вьполнено условие Миттаг-Лефффлера. В самом деле, пусть $X_{n}$ - свободный $\mathbb{Z}[\mathbb{Z} / 2]$-модуль с $\left(3^{n}+1\right) / 2$ образуюшей $a_{0}, \ldots, a_{\left(3^{n}-1\right) / 2}$. Заметим, что имеет место равенство $\left(3^{n+1}-1\right) / 2=1+3+9+\cdots+3^{n}=1+3\left(3^{n}-1\right) / 2$. Определим $\pi: X_{n+1} \rightarrow X_{n}$ посредством $a_{i} \mapsto a_{[i+1 / 3]}$, так что $a_{0} \in X_{n}$ имеет прообразом две, а все остальные $a_{i} \in X_{n}$-по три образующие $a_{j} \in X_{n+1}$. Наконец, зададим модульный гомоморфизм $\varphi_{n}: \mathbb{Z}[\mathbb{Z} / 2] \rightarrow X_{n}$, переводя образующую в $a_{0}+(1+t)\left(a_{1}+a_{2}+\cdots+a_{\left(3^{n}-1\right) / 2}\right)$. Легко видеть, что мономорфизмы $\varphi_{n}$ коммутируют со спектральньми проекциями $\pi_{i}$ и $a \mapsto 2 a+t a$.
} 
ТЕОРема 6.1. Пусть $X^{n}$ - компактный полиэдр, $M^{m-k}$ - ориентируемое PL-многообразие и $\bar{f}: X \stackrel{f}{\longrightarrow} M \subset M \times \mathbb{R}^{k}$ реализуемо дискретно, но $\vartheta(\bar{f}) \neq 0$. Если нить ван Кампена-Скопенкова $\check{\vartheta}(f) \in \lim H_{\mathrm{skew}}^{m-k}\left(\widetilde{X}, U_{i}\right)$ является элементом конечного порядка, то ее элементы ч-делятся на класс Эйлера е(t).

Случай $k=1$ рассмотрен вьше, а при $k \geqslant 2$ сложность представляет лишш установление факта, что, если порядок $\check{\vartheta}(f)$ конечен, он непременно равен 2.

ЛЕмма 6.2. В условиях теоремы 6.1 предположим, что $k \geqslant 2$. Если $\check{\vartheta}(f)$ имеет порядок 2, то каждый ее әлемент $\vartheta_{i}(f)$ делится на класс Эйлера е(t).

ДокаЗАтельство. Рассмотрим последовательности Смита

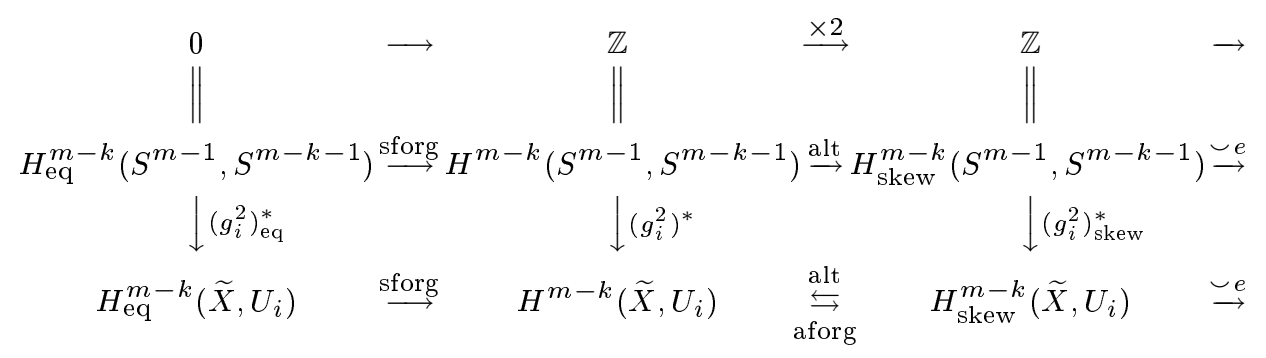

По условию каждый $\vartheta_{i}(f)=\left(g_{i}^{2}\right)_{\text {skew }}^{*}\left(\xi_{\text {skew }}\right)$ имеет порядок 2 и ввиду $k \geqslant 2$ нетривиально умножается на класс Эйлера согласно лемме $3.3,(\mathrm{a})$ и наблюдению 3.4. Значит, $\operatorname{alt}\left(g_{i}^{2}\right)^{*}(\xi)=\left(g_{i}^{2}\right)_{\text {skew }}^{*}\left(2 \xi_{\text {skew }}\right)=0$, где $\xi=\operatorname{aforg} \xi_{\text {skew }}$. Однако так как $k \geqslant 2$, группа $H_{\mathrm{eq}}^{m-k}\left(S^{m-1}, S^{m-k-1}\right)$ тривиальна, значит, в обра-

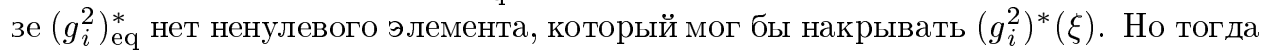
$\operatorname{aforg} \vartheta_{i}(f)=\left(g_{i}^{2}\right)^{*}\left(\operatorname{aforg} \xi_{\text {skew }}\right)=0$, поэтому каждый $\vartheta_{i}(f)$ обязан приходить из $H_{\text {skew }}^{m-1}\left(\widetilde{X}, U_{i}\right)$ при гомоморфизме умножения на класс Эйлера.

Нам потребуются некоторые построения. Пусть $K$ и $L-$ полиэдры со свободньгми кусочно линейньпи действиями $\mathbb{Z} / 2=\left\langle\mathfrak{t} \mid \mathfrak{t}^{2}=1\right\rangle$. Через $K \times_{\mathbb{Z} / 2} L$ будем обозначать факторпространство $K \times L$ по диагональному действию $\mathbb{Z} / 2$; на нем, в свою очередь, задано каноническое действие $\mathbb{Z} / 2$, переводящее $(x, y)=(\mathfrak{t} x, \mathfrak{t} y)$ в $(x, \mathfrak{t} y)=(\mathfrak{t} x, y)$, так что $\left(K \times_{\mathbb{Z} / 2} L\right) / \mathfrak{t} \cong(K / \mathfrak{t}) \times(L / \mathfrak{t})$. Можно показать, что класс Эйлера двулистного накрытия $p: K \times_{\mathbb{Z} / 2} L \rightarrow(K / \mathfrak{t}) \times(L / \mathfrak{t})$ отождествляется с периодическим произведением классов Эйлера двулистных накрытий $K \rightarrow K / \mathfrak{t}$ и $L \rightarrow L / \mathfrak{t}$, но это нам не потребуется. Композиция накрытия $p$ и проекции доставляет локально тривиальное расслоение $K \times_{\mathbb{Z} / 2} L \rightarrow K / \mathfrak{t}$ со слоем $L$, причем аналогичный факт имеет место и в случае, если действие $\mathbb{Z} / 2$ на $L$ имеет неподвижные точки. В случае $L=S^{k-1}$ с антиподальным действием $\mathbb{Z} / 2$ это расслоение $\widehat{\nu}_{k}: K \times_{\mathbb{Z} / 2} S^{k-1} \rightarrow K / \mathfrak{t}$, при $k=\infty$ известное как конструкция Бореля, может быть описано как джойн Уитни $k$ экземпляров двулистного накрытия $\nu: K \rightarrow K / \mathfrak{t}$ $[27 ; \S 6]$, т.е. сферизация суммы Уитни $k$ экземпляров линейного расслоения, ассоциированного с $\nu$. Как подметил Хэфлигер, сушествование сечения в этом расслоении равносильно существованию эквивариантного отображения $K \rightarrow S^{k-1}$. Для расслоения $\widehat{\nu}_{k}$ и произвольного локально постоянного пучка $\mathscr{O}$ на $K / \mathfrak{t}$ имеется точная последовательность Смита-Гизина [22]:

$$
\begin{aligned}
H^{d}(K / \mathfrak{t} ; \mathscr{O}) \stackrel{p^{*}}{\longrightarrow} H^{d}\left(K \times_{\mathbb{Z} / 2} S^{k-1} ; \widehat{\nu}_{k}^{*} \mathscr{O}\right) \stackrel{p^{!}}{\longrightarrow} H^{d+1-k}(K / \mathfrak{t} ; \mathscr{O} \otimes \mathscr{L}) \\
\stackrel{\smile e^{k}}{\longrightarrow} H^{d+1}(K / \mathfrak{t} ; \mathscr{O}) .
\end{aligned}
$$


Здесь $\mathscr{L}-(k-1)$-мерный пучок Лере расслоения $\widehat{\nu}_{k}$, т.е. $\mathscr{O}_{\mathbb{Z}_{\mathrm{T}}^{\otimes k}}=\mathscr{O}_{\mathbb{Z}_{\mathrm{T}}}^{\otimes k}$, а $\widehat{\nu}_{k}^{*} \mathscr{O}$ - обратньй образ пучка $\mathscr{O} ;$ наконец, $e^{k} \in H^{k}(K / \mathfrak{t} ; \mathscr{L})$ - класс Эйлера расслоения $\widehat{\nu}_{k}$, равный по формуле Уитни $k$-й $\smile$-степени класса Эйлера $e$ двулистного накрытия $\nu$. Гомоморфизм $p^{*}$ индуцирован проекцией на базу, а гомоморфизм Гизина $p^{!}$соответствует связываюшему гомоморфизму $\delta^{*}$ из точной последовательности пары $\left(K \times_{\mathbb{Z} / 2} B^{k}, K \times_{\mathbb{Z} / 2} S^{k-1}\right)$ при изоморфизме Тома

$$
H^{d+1}(K / \mathfrak{t} ; \mathscr{O} \otimes \mathscr{L}) \simeq H^{d+1-k}\left(K \times_{\mathbb{Z} / 2} B^{k}, K \times_{\mathbb{Z} / 2} S^{k-1} ; \widehat{\nu}_{k}^{*} \mathscr{O}\right) .
$$

Напомним, что $p^{!}$можно также получить из индуцированного проекцией на базу гомоморфизма в гомологиях с помощью двойственности Пуанкаре (если $K-$ многообразие) и с помошью послойного интегрирования в теории де Рама (если $K$ - гладкое многообразие), а тот факт, что умножение на $e^{k}$ отождествляется с гомоморфизмом расширения носителя из точной последовательности пары $\left(K \times_{\mathbb{Z} / 2} B^{k}, K \times_{\mathbb{Z} / 2} S^{k-1}\right)$, вытекает из того, что класс Тома расслоения $\widehat{\nu}_{k}$ переходит при этом гомоморфизме в его класс Эйлера. Зафиксируем $\mathscr{O}=\mathscr{O}_{\mathbb{Z}_{\mathrm{T}}}$ (относительно действия $\mathbb{Z} / 2$ на $K$ ), тогда $\widehat{\nu}^{*} \mathscr{O}$ есть 0 -мерный пучок Лере накрытия $K \times S^{k-1} \rightarrow K \times_{\mathbb{Z} / 2} S^{k-1}$, т.е. $\mathscr{O}_{\mathbb{Z}_{\mathrm{T}}}$ (относительно диагонального действия $\mathbb{Z} / 2$ на $\left.K \times S^{k-1}\right)$, и в терминах модулей последовательность Смита-Гизина относительно подполиэдра $L \subset K$ приобретает вид

$$
\begin{aligned}
& \cdots \rightarrow H_{\mathrm{eq}}^{d}(K, L) \stackrel{p^{*}}{\longrightarrow} H_{\mathrm{eq}}^{d}\left(K \times S^{k-1}, L \times S^{k-1}\right) \stackrel{p^{!}}{\longrightarrow} H_{\text {skew }}^{d-k+1}(K, L) \\
& \stackrel{\smile e^{k}}{\longrightarrow} H_{\text {skew }}^{d+1}(K, L) \stackrel{p^{*}}{\longrightarrow} H_{\text {skew }}^{d+1}\left(K \times S^{k-1}, L \times S^{k-1}\right) \stackrel{p^{!}}{\longrightarrow} H_{\mathrm{eq}}^{d-k+2}(K, L) \stackrel{\smile e^{k}}{\longrightarrow} \cdots .
\end{aligned}
$$

Следуюшая лемма понадобится нам по сушеству лишв в случае $P=\varnothing$.

Лемма 6.3. Рассмотрим әквивариантное отображение $\varphi: P \rightarrow S^{k-l-1}$ инвариантного подполиэдра $P \stackrel{\iota}{\subset} K$ и соответствующее частичное сечение $s=\iota \times \varphi: P \rightarrow K \times S^{k-l-1}$, a также еще один инвариантный подполиэдр $L$ полиәдра $K$. Включения $K \times_{\mathbb{Z} / 2} S^{l-1} \stackrel{\mathfrak{i}}{\subset} K \times_{\mathbb{Z} / 2} S^{k-1} \stackrel{\mathfrak{j}}{\zeta} K \times_{\mathbb{Z} / 2} S^{k-l-1}$, соответствующие разложению $S^{k-1}=S^{l-1} * S^{k-l-1}$, описвьваются четырвмя точныци последовательностями, образующими коммутативную диаграмму

$$
\begin{aligned}
& \stackrel{p^{*}}{\stackrel{\mathfrak{i}^{*}}{\rightarrow}} H_{\text {skew }}^{d-1}\left(K \times S^{l-1},(P \cup L) \times S^{l-1}\right) \\
& \downarrow p^{!} \quad \searrow^{\delta^{*}} \\
& \stackrel{-e^{k-l}}{\rightarrow} H_{\mathrm{eq}}^{d-l}(K, P \cup L) \stackrel{p^{*}}{\rightarrow} H_{\mathrm{eq}}^{d-l}\left(K \times S^{k-l-1}, s(P) \cup L \times S^{k-l-1}\right) \\
& \searrow e^{k} \quad \downarrow \cup e^{l} \quad \downarrow j ! \\
& H_{\mathrm{eq}}^{d}(K, P \cup L) \quad \stackrel{p^{*}}{\rightarrow} H_{\mathrm{eq}}^{d}\left(K \times S^{k-1}, s(P) \cup L \times S^{k-1}\right) \stackrel{p^{!}}{\rightarrow} H_{\text {skew }}^{d-k+1}(K, L) \\
& \searrow^{p^{*}} \quad \downarrow \mathfrak{i}^{*} \quad \downarrow \downarrow e^{k-l} \quad \searrow^{k} \\
& H_{\mathrm{eq}}^{d}\left(K \times S^{l-1},(P \cup L) \times S^{l-1}\right) \quad \stackrel{p^{\prime}}{\rightarrow} H_{\mathrm{skew}}^{d-l+1}(K, P \cup L) \stackrel{\smile e^{l}}{\rightarrow} \\
& \searrow^{\delta^{*}} \downarrow p^{*} \\
& H_{\text {skew }}^{d-l+1}\left(K \times S^{k-l-1}, s(P) \cup L \times S^{k-l-1}\right) \stackrel{\mathfrak{j}^{!}}{\rightarrow} \\
& p^{!}
\end{aligned}
$$


В этой диаграмме некоторые гомоморфизмы вполне соответствуют своим обозначениям лишь в случае $P=\varnothing$, а в общем случае определены в доказательстве. Гомоморфизм j! соответствует при двойственности Пуанкаре (если $K$ - многообразие) индуцированному включением гомоморфизму $\mathfrak{j}_{*}$ в гомологиях, а в обшем случае (при $P=\varnothing$ ) получается с помощью изоморфизма Тома

$$
H_{\mathrm{eq}}^{d-l}\left(K \times S^{k-l-1}, L \times S^{k-l-1}\right) \cong H_{\mathrm{eq}}^{d}\left(K \times S^{k-1}, K \times S^{l-1} \cup L \times S^{k-1}\right)
$$

из гомоморфизма расширения носителя в точной последовательности тройки; гомоморфизм $\delta^{*}$ получается из связьваюшего гомоморфизма в последней.

ДокАЗАтЕльство. Рассмотрим точные последовательности троек, выбранных из четверки

$$
\left(K \times B^{k}, K \times S^{k-1} \cup Z, K \times S^{l-1} \cup Z, Z\right),
$$

где $Z=\widehat{P} \cup L \times B^{k}$, а $\widehat{P} \subset P \times B^{k}$ - послойньй джойн $s(P)$ и $P \times B^{l}$, пересекающий каждый слой $p t \times B^{k}=p t \times\left(S^{k-l-1} * B^{l}\right)$ по $(l+1)$-мерному шару. Легко видеть, что предъявленная диаграмма (за исключением стрелок, еще не определенных в случае непустого $P$ ) изоморфна стандартной диаграмме (cp. [42; диаграмма в п. 3.19]), образованной этими последовательностями, что и позволяет доопределить все стрелки в случае непустого $P$. Отметим лишь, что умножение на $e^{k-l}$ доопределяется умножением на класс $E \in H_{\mathrm{skew}}^{k-l}(K, P)$, представленный нулями произвольного эквивариантного сечения $\bar{s}: K \rightarrow K \times B^{k-l} \backslash \widehat{P}$, продолжающего $\mathfrak{t} s$.

Возвращаясь к обозначениям из формулировки теоремы 6.1 , рассмотрим последовательность Смита-Гизина для пары $\left(\widetilde{X}, U_{i}\right)$ :

$$
H_{\mathrm{eq}}^{m-1}\left(\widetilde{X}, U_{i}\right) \stackrel{p^{*}}{\longrightarrow} H_{\mathrm{eq}}^{m-1}\left(\widetilde{X} \times S^{k-1}, U_{i} \times S^{k-1}\right) \stackrel{p^{!}}{\longrightarrow} H_{\mathrm{skew}}^{m-k}\left(\widetilde{X}, U_{i}\right) \stackrel{e^{k}}{\longrightarrow}
$$

ПРЕДЛОЖЕНИЕ 6.4. В условиях теоремы 6.1 обозначим $Q^{m}=M \times \mathbb{R}^{k}, u$ для каждого $i$ пусть $g_{i}: X \hookrightarrow Q$ - вложение, $2^{-i}$-близкое $\kappa \bar{f}$. Система, коэффициенты которой получень из коэффициентов $d_{\mathrm{eq}}\left(g_{i}, g_{i+1}\right)$ системь (1) с помощью гомоморфизмов $p^{*}$, разрешима. Более того, справедлива формула $\vartheta(\bar{f})=h^{-1} \delta^{*}(\check{\vartheta}(f))$, где $h-$ изоморфизм из леммы 4.3, а

$$
\delta^{*}: \underset{\lim }{\longleftarrow}\left[\operatorname{ker} e^{k} \mid H_{\mathrm{skew}}^{m-k}\left(\widetilde{X}, U_{i}\right)\right] \rightarrow \lim ^{1}\left[H_{\mathrm{eq}}^{m-1}\left(\widetilde{X}, U_{i}\right) / \operatorname{im} e^{k}\right]
$$

- связьвающий гомоморфизм из шестичленной последовательности (2), построенной по короткой точной последовательности обратных последовательностей

$$
H_{\mathrm{eq}}^{m-1}\left(\widetilde{X}, U_{i}\right) / \operatorname{im} e^{k} \longmapsto H_{\mathrm{eq}}^{m-1}\left(\widetilde{X} \times S^{k-1}, U_{i} \times S^{k-1}\right) \rightarrow \operatorname{ker} e^{k} \mid H_{\mathrm{skew}}^{m-k}\left(\widetilde{X}, U_{i}\right)
$$

ДокАЗАтЕльство. Отображение $g_{i}^{2}: \widetilde{X} \rightarrow \widetilde{Q}$ дает $\widehat{g}_{i}: \widetilde{X} \times S^{k-1} \rightarrow \widetilde{Q} \times S^{k-1}$. Поскольку $g_{i}$ является $2^{-i}$-близким к $\frac{i}{f}$, образ ограничения $g_{i}^{2}$ на $U_{i}$ содержится вблизи $\widetilde{M}$, так что $\widehat{g}_{i}\left(U_{i} \times S^{k-1}\right)$ лежит в малой окрестности $\widetilde{M} \times S^{k-1}$. Поэтому достаточно установить существование класса $\widehat{\xi} \in H_{\mathrm{eq}}^{m-1}\left(\widetilde{Q} \times S^{k-1}, \widetilde{M} \times S^{k-1}\right)$ 
такого, что $p^{!}(\widehat{\xi})$ совпадает с классом $\xi_{\text {skew }} \in H_{\text {skew }}^{m-k}(\widetilde{Q}, \widetilde{M})$ из леммы $3.3,($ в) и имеет место тождество

$$
p^{*} d_{\mathrm{eq}}\left(g_{i}, g_{i+1}\right)=\widehat{g}_{i+1}^{*}(\widehat{\xi})-\widehat{g}_{i}^{*}(\widehat{\xi}) .
$$

Пусть $\widehat{\xi}$ - произвольный класс, удовлетворяющий первому условию, существующий в силу $\xi_{\text {skew }} \smile e^{k}=0$; покажем, что он удовлетворяет и второму (с незначительной поправкой). Для этого рассмотрим следующую диаграмму, полученную из леммы 6.3 при $l=1, K=\widetilde{Q}, L=\widetilde{M}$ и $P=\varnothing$ :

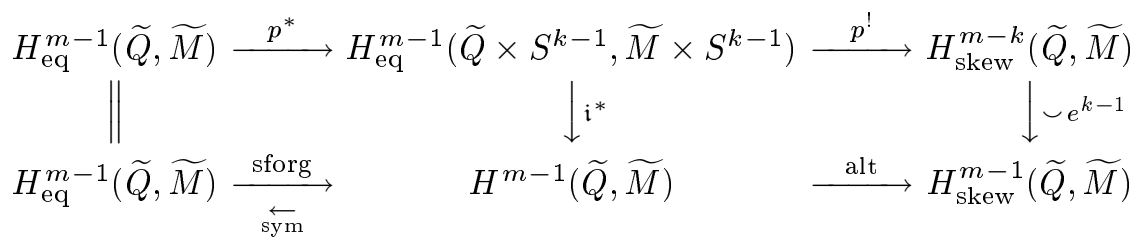

По лемме $3.3,(\mathrm{a})\left(g_{i}^{2}\right)_{\text {skew }}^{*}\left(\xi_{\text {skew }}\right)=\vartheta_{i}(f)$ для каждого $i$, значит, поскольку классы $\vartheta_{i}(f)$ образуют нить,

$$
\left(g_{i+1}^{2}\right)_{\text {skew }}^{*}\left(\xi_{\text {skew }}\right)-\left(g_{i}^{2}\right)_{\text {skew }}^{*}\left(\xi_{\text {skew }}\right)=0
$$

в группе $H_{\mathrm{skew}}^{m-k}\left(\widetilde{X}, U_{i}\right)$. Следовательно, класс $\widehat{d}_{i}:=\widehat{g}_{i+1}^{*}(\widehat{\xi})-\widehat{g}_{i}^{*}(\widehat{\xi})$ лежит в образе $p^{*} ;$ пусть $d_{i} \in H_{\mathrm{eq}}^{m-1}\left(\widetilde{X}, U_{i}\right)$ - какой-нибудь его прообраз. Поскольку $\mathfrak{i}^{*} p^{*}=$ sforg, композиция sym $i^{*} p^{*}$ совпадает с умножением на 2 в группе $H_{\mathrm{eq}}^{m-1}\left(\widetilde{X}, U_{i}\right)$, в частности, $\operatorname{symi}^{*}\left(\widehat{d}_{i}\right)=2 d_{i}$. Однако $\operatorname{sym}^{*}(\widehat{\xi})=\xi_{\text {eq }} \in H_{\mathrm{eq}}^{m-1}(\widetilde{Q}, \widetilde{M})$, поэтому

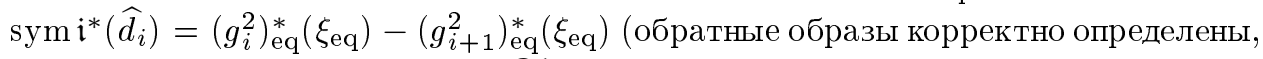
поскольку $g^{2}\left(U_{i}\right)$ лежит вблизи $\left.\widetilde{M}\right)$, а последняя разность равна $2 d_{\mathrm{eq}}\left(g_{i}, g_{i+1}\right)$ по лемме $2.3,(\mathrm{a})$. Следовательно, $d_{i}=d_{\mathrm{eq}}\left(g_{i}, g_{i+1}\right)+\varepsilon_{i}$, где $\varepsilon_{i}$ - элемент порядка 2 . Отсюда и $\widehat{d}_{i}=p^{*} d_{\text {eq }}\left(g_{i}, g_{i+1}\right)+\widehat{\varepsilon}_{i}$, где $\widehat{\varepsilon}_{i}=p^{*}\left(\varepsilon_{i}\right)$, т.е. равенство $(7)$ доказано с точностью до элемента $\widehat{\varepsilon}_{i}$ порядка 2 . Поскольку в произвольной обратной последовательности из конечных групп выполнено условие Миттаг-Леффлера, поправки $\widehat{\varepsilon}_{i}$ не влияют на разрешимость $p^{*}$-образа системы (1), а добавки $\varepsilon_{i}-$ на класс системы (1) в производном пределе.

ЗАМЕчАНИЕ. Поскольку $\mathfrak{i}^{*}\left(\widehat{\varepsilon}_{i}\right)=\operatorname{sforg}\left(\varepsilon_{i}\right)=0$ ввиду того, что при $k=1$ равенство (7) выполнено без поправок по лемме $2.3,\left(\right.$ б), класс $\varepsilon_{i}$ делится на класс Эйлера $е$. Неясно, однако, может ли $\varepsilon_{i}$ оказаться ненулевым.

ЗАмЕчАниЕ. Вышеприведенное доказательство может быть мотивировано следующей коммутативной диаграммой, в которой верхняя строчка - интересующая нас последовательность Смита-Гизина (для краткости $m-1$ заменено на $d$ ), нижняя строчка - аналогичная последовательность для $k=1$, рассматривавшаяся в $\S 4$, а все вертикальные стрелки - изоморфизмы (в среднем столбце - модульные над $\mathbb{Z}[\mathbb{Z} / 2])$ :

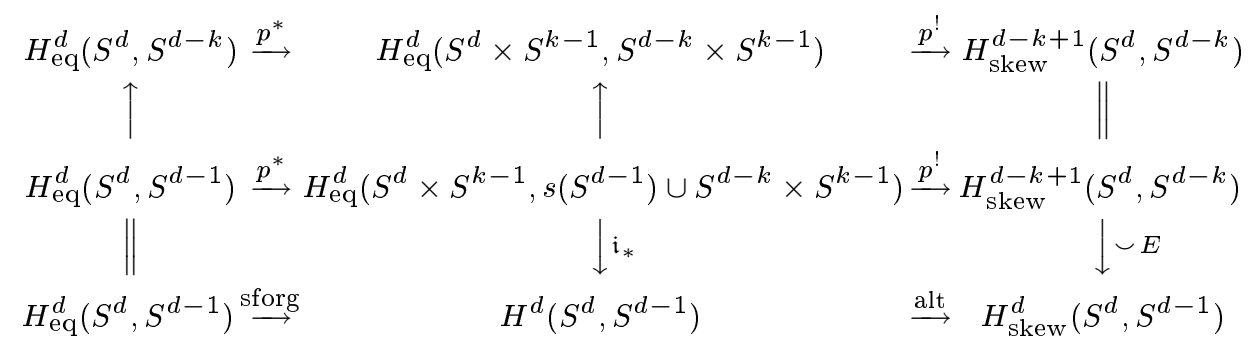


Нижние две строчки получены из леммы $6.3 \mathrm{c} l=1, K=S^{d}$ и регулярными окрестностями $S^{d-k}$ и $S^{k-2}$ в $S^{d}$ в качестве $L$ и $P$ соответственно, достаточно большими для того, чтобы их объединение было регулярной окрестностью $S^{d-1}=$ $S^{d-k} * S^{k-2}$.

ДоКАЗАТЕЛЬСТво теОРемЫ 6.1. Согласно случаям $l=1$ и $l=k-1$ леммы 6.3 (в обоих случаях $P=\varnothing$ ) имеет место следующая диаграмма, в которой коммутативна часть, образованная стрелками sforg и alt:

$$
\begin{aligned}
& H_{\mathrm{eq}}^{m-2}\left(\widetilde{X}, U_{i}\right) \stackrel{-e}{\longrightarrow} \quad H_{\mathrm{eq}}^{m-1}\left(\widetilde{X}, U_{i}\right) \underset{\text { sforg }}{\stackrel{\mathrm{sym}}{\leftrightarrows}} H^{m-1}\left(\widetilde{X}, U_{i}\right) \\
& p_{i}^{*} \quad \nearrow \mathrm{i}_{i}^{*} \\
& H_{\mathrm{eq}}^{m-1}\left(\widetilde{X} \times S^{k-1}, U_{i} \times S^{k-1}\right) \\
& p_{i}^{!} \downarrow \quad \nwarrow i ! \\
& H_{\mathrm{skew}}^{m-k-1}\left(\widetilde{X}, U_{i}\right) \stackrel{\smile e}{\longrightarrow} \quad H_{\mathrm{skew}}^{m-k}\left(\widetilde{X}, U_{i}\right) \underset{\text { aforg }}{\stackrel{\text { alt }}{\leftrightarrows}} H^{m-k}\left(\widetilde{X}, U_{i}\right)
\end{aligned}
$$

Обозначим через $\tau_{i}^{*}$ и $\tau_{i}^{!}$"трансферы” sym $\circ \mathfrak{i}_{i}^{*}$ и $\mathfrak{i}_{i}^{!} \circ \operatorname{aforg}$ соответственно. Из свойств (i) и (ii) из $\S 4$ следует, что композиции $\tau_{i}^{*} \circ p_{i}^{*}$ и $p_{i}^{!} \circ \tau_{i}^{!}$являются эндоморфизмами умножения на 2.

Как отмечалось выше, на тотальном пространстве $\widetilde{X} \times_{\mathbb{Z} / 2} S^{k-1}$ расслоения $\widehat{\nu}_{k}$ определена инволюция, ограничение которой на каждый его слой $S^{k-1}$ дает антиподальную инволюцию, причем $\left(\widetilde{X} \times_{\mathbb{Z} / 2} S^{k-1}\right) / \mathfrak{t} \cong(\widetilde{X} / \mathfrak{t}) \times \mathbb{R} P^{k-1}$. Отображения $\widehat{g}_{i}: X \times_{\mathbb{Z} / 2} S^{k-1} \rightarrow \widetilde{Q} \times_{\mathbb{Z} / 2} S^{k-1}$, получаемые из $g_{i}^{2}$, эквивариантны относительно этих инволюций, причем в случае $Q=\mathbb{R}^{m}$ инволюция на $S^{m-1} \times_{\mathbb{Z} / 2} S^{k-1}$ преврашает группу $H_{\mathrm{eq}}^{m-1}\left(S^{m-1} \times S^{k-1}, S^{m-k-1} \times S^{k-1}\right)$ в свободный циклический модуль над $\mathbb{Z}[\mathbb{Z} / 2]$. В обшем случае свободный циклический подмодуль в $\mathbb{Z}[\mathbb{Z} / 2]$-модуле $H_{\mathrm{eq}}^{m-1}\left(\widetilde{Q} \times S^{k-1}, \widetilde{M} \times S^{k-1}\right)$ порождается элементом $\widehat{\xi}$, определенным в доказательстве предложения 6.4. Теперь несложно проверить, что доказательство альтернативы 5.2 с заменой гомоморфизмов sforg, alt, sym, aforg coответственно на $p_{i}^{*}, p_{i}^{!}, \tau_{i}^{*}, \tau_{i}^{!}$показывает, что $\vartheta_{i}(f)$ - элемент порядка 2 , что и требовалось.

ЗАмечАниЕ. Отметим, что гомоморфизм $\tau_{i}^{*}$ не совпадает, вообще говоря, с трансфером Беккера-Готтлиба [43]. Действительно, в ориентируемом случае ( $k$ четно, $m$ четно) мы попадаем в классическую ситуацию (все пучки тривиальны), и композиция гомоморфизма $p^{*}$ и трансфера Беккера-Готтлиба является умножением на эйлерову характеристику слоя, т.е. нулевьм гомоморфизмом.

ДОКАЗАТЕЛЬСТвО ТЕОРЕМЫ 2. (а) По теореме 6.1 и предложению 4.6 группа $\lim ^{1} H_{\mathrm{eq}}^{2 n-1}\left(\widetilde{S^{n}}, \widetilde{S^{n} \backslash R_{i}}\right)$ содержит элемент порядка 2, где $R_{i}-$ замкнутая инвариантная полиэдральная окрестность $S_{x}=\left(P_{x} \times\{x\} \cup\{x\} \times P_{x}\right) \backslash\{(x, x)\}$ в $\widetilde{S^{n}}$, лежашая в $2^{-i}$-окрестности и содержащая $2^{-i-1}$-окрестность. По двойственности Александера $H_{\mathrm{eq}}^{2 n-1}\left(\widetilde{S^{n}}, \widetilde{S^{n}} \backslash R_{i}\right) \cong H_{1}^{\mathbb{Z} / 2 ; \text { lf }}\left(R_{i} ; \mathbb{Z}\right)$, где действие $\mathbb{Z} / 2$ на коэффициентах тривиально, так что последняя группа есть просто $H_{1}^{\mathrm{lf}}\left(R_{i} / \mathfrak{t}\right)$. Требуемое утверждение следует теперь из точности последовательности Милнора (см. [5; 
приложение])

$$
0 \rightarrow \lim ^{1} H_{1}^{\mathrm{lf}}\left(R_{i} / \mathfrak{t}\right) \rightarrow H_{0}^{\mathrm{lf}}\left(S_{x} / \mathfrak{t}\right) \stackrel{\mathscr{F}}{\longrightarrow} \check{H}_{0}^{\mathrm{lf}}\left(S_{x} / \mathfrak{t}\right) \rightarrow 0
$$

так как $S_{x} / \mathfrak{t} \cong P_{x} \backslash\{x\}$, локально конечные гомологии которого (чеховские или стинродовские) изоморфны приведенньм гомологиям $P_{x}$.

(б) Из лемм 4.1 и 4.2 следует, что класс $\vartheta_{\varepsilon}\left(\bar{f} \times \mathrm{id}_{[0,1)}\right)$ лежит в образе группы $H_{\text {skew }}^{2 n+1}\left(\widetilde{X} \times[0,1), \widetilde{X} \times[0,1) \backslash S_{x} \times[0,1)\right)$. Доказательство наблюдения 3.2 показывает, что $\vartheta(\bar{f})$ лежит тогда в образе $H_{\text {skew }}^{2 n}\left(\widetilde{X}, \widetilde{X} \backslash S_{x}\right)$. Двойственность Александеpa (cp. [5; док-во теоремы $2,(\mathrm{a})])$ позволяет утверждать, что $o(\bar{f})$ лежит в образе $H_{0}^{\mathbb{Z} / 2 ; \text { lf }}\left(S_{x} ; \mathbb{Z}\right)$, где $\mathbb{Z} / 2$ действует на коэффициентах тривиально, так что последняя группа изоморфна $H_{0}^{\mathrm{lf}}\left(P_{x} \backslash\{x\}\right)$.

Автор признателен П.М. Ахметьеву, А.Н. Дранишникову, Ю.Б. Рудяку, Е.Г. Скляренко, К.Э. Фельдману, А.В. Чернавскому и Е.В. Шепину за внимание к работе и стимулирующие обсуждения. Требования рецензента по переоформлению статьи повлекли усиление результатов и упрощение доказательств.

\section{Список литературы}

1. Siektucki K. Realization of mappings // Fund. Math. 1969. V. 65. № 3. P. 325-343.

2. Щепин E. B., Штанько M. A. Спектральный критерий вложимости компактов в евклидово пространство // Труды Ленинградской международной топологической конференции. Л.: Наука, 1983. С. 135-142.

3. Ахметьев П. М. Об изотопической и дискретной реализации отображений $n$-сферы в евклидовом пространстве // Матем. сб. 1996. Т. 187. № 7. С. 3-34.

4. Mekikhov S. A. On maps with unstable singularities // Topology Appl. 2002. V. 120. P. $105-156$.

5. Мелихов C.A. Изотопическая и непрерьвная реализуемость отображений в метастабильном ранге // Матем. сб. 2004. Т. 195. № 7. С. 71-104.

6. Ахметьев П. М. Вложения компактов, стабильные гомотопические группы сфер и теория особенностей // УМН. 2000. Т. 55. №3. С. 3-62.

7. Ахметьев П. М., Мелихов C. А. Об изотопической реализуемости непрерьвных отображений / / Записки науч. сем. ПОМИ. 2000. Т. 267. С. 53-87; Исправление (в печати); исправление учтено в пер. на англ.: J. Math. Sci. (New York). 2003. V. 113. P. 759-776.

8. Мелихов C. A., Михайлов P. В. Зацепления по модулю узлов и проблема изотопической реализации // УМН. 2001. Т. 56. № 2. С. 219-220.

9. Melikhov S. A., Repovš D. n-quasi-isotopy. I: Questions of nilpotence // J. Knot Theory Ramifications. (to appear).

10. Akhmetiev P. M., Szücs A. Geometric proof of the easy part of the Hopf invariant one theorem // Math. Slovaca 1999. V. 49. № 1. P. 71-74.

11. Melikhov S. A. Sphere eversions and realization of mappings // math.GT/0305158.

12. Akhmet'ev P.M., Repov̌s D., Skopenkov A.B. Obstructions to approximating maps of $n$-manifolds into $\mathbb{R}^{2 n}$ by embeddings // Topology Appl. 2002. V. 123. P. 3-14.

13. Zeeman E. C. Unknotting combinatorial balls // Ann. of Math. (2). 1963. V. 78. P. 501-526.

14. Edwards R. D. The equivalence of close piecewise-linear embeddings // General Topology Appl. 1975. V. 5. P. 147-180.

15. Koschorke U., Sanderson B. Geometric interpretations of the generalized Hopf invariant // Math. Scand. 1977. V. 41. P. 199-217.

16. Akhmetiev P. M. Pontrjagin-Thom construction for approximation of mappings by embeddings // Topology Appl. (to appear).

17. Adem J. The iteration of the Steenrod squares in algebraic topology // Proc. Nat. Acad. Sci. USA 1952. V. 38. № 8. P. 720-726.

18. Ахметьев П. М. Геометрический подход к стабильным гомотопическим группам сфер. Инварианты Хопфа-Адамса // Изв. РАН. Сер. матем. (в печати). 
19. Repovš D., Skopenkov A. B. A deleted product criterion for approximability of maps by embeddings // Topology Appl. 1998. V. 87. P. 1-19.

20. Yang C.-T. p-adic transformation groups // Michigan Math. J. 1960. V. 7. P. 201-218.

21. Маклейн С. Гомология. М.: Мир, 1966.

22. Бредон Г. Теория пучков. М.: Наука, 1988.

23. Масси У. Теория гомологий и когомологий. М.: Мир, 1981.

24. Репови Д., Скопенков А. Б. Новые результаты о вложениях полиэдров и многообразий в евклидовы пространства // УМН. 1999. Т. 54. №6. С. 61-108.

25. Krushkal V.S. Embedding obstructions and 4-dimensional thickenings of 2-complexes // Proc. Amer. Math. Soc. 2000. V. 128. P. 3683-3691.

26. Bestvina M., Kapovich M., Kleiner B. Van Kampen's embedding obstruction for discrete groups // Invent. Math. 2002. V. 150. P. 219-235.

27. Conner P.E., Floyd E. E. Fixed point free involutions and equivariant maps // Bull. Amer. Math. Soc. 1960. V. 66. №6. P. 416-441.

28. Geoghegan $R$. A note on the vanishing of $\lim ^{1} / /$ J. Pure Appl. Algebra. 1980. V. 17. P. 113-116.

29. Jensen C. U. Les foncteurs dérives de lim et leurs applications en théorie des modules. Berlin: Springer-Verlag, 1972. (Lecture Notes in Math. V. 254.)

30. Hughes B., Ranicki A. Ends of complexes. Cambridge: Cambridge Univ. Press, 1996. (Cambridge Tracts in Math. V. 123.)

31. Кузьминов В. И. О производных функторах функтора проективного предела // Сиб. матем. журн. 1967. Т. 8. №3. С. 333-345.

32. Gray B. I. Spaces of the same $n$-type, for all $n / /$ Topology. 1966. V. 5. P. 241-243.

33. Харлап A. Э. Локальные гомологии и когомологии, гомологическая размерность и обобщенные многообразия // Матем. сб. 1975. Т. 96. № 3. С. 347-373.

34. Гийу Л., Марен А. Комментарии к четырем статьям В. А. Рохлина: II. О третьей статье // В поисках утраченной топологии. М.: Мир, 1989. С. 65-92.

35. Miller J. G. Self-intersections of some immersed manifolds // Trans. Amer. Math. Soc. 1969. V. 136. P. 329-338.

36. Repovš D., Skopenkov A. B. On projected embeddings and desuspending the $\alpha$-invariant // Topology Appl. 2002. V. 124. P. 69-75.

37. Massey W. On the Stiefel-Whitney classes of a manifold // Amer J. Math. 1960. V. 82. P. 92-102.

38. Громов $М$. Дифференциальные соотношения с частными производными. М.: Мир, 1990.

39. Rourke C. P., Sanderson B. J. The compression theorem. I // Geom. Topol. 2001. V. 5. P. 399-429.

40. Edwards D. A., Hastings H. M. Čech and Steenrod homotopy theories with applications to geometric topology. Berlin: Springer-Verlag, 1976. (Lecture Notes in Math. V. 542.)

41. Фукс Л. Бесконечные абелевы групшы. Т. 1. М.: Мир, 1974.

42. Свитцер P. М. Алгебраическая топология - гомотопии и гомологии. М.: Наука, 1985.

43. Gottlieb D. H. Fiber bundles and the Euler characteristic // J. Differential Geom. 1975. V. 10. № 1. P. 39-48.

Математический институт им. В.А. Стеклова;

University of Florida, USA

E-mail: melikhov@mi.ras.ru

melikhov@math.ufl.edu
Поступила в редакцию 26.08 .2002 и 12.01 .2004 